ARL 66-0029

\title{
HYDROSTATIC TENSILE FRACTURE OF A POLYURETHANE ELASTOMER
}

\author{
GERALD H. LINDSEY \\ CALIFORNIA INSTITUTE OF TECHNOLOGY \\ PASADENA, CALIFORNIA
}

FEBRUARY 1966

Contract AF 33(615)-2217

Project 7063

AEROSPACE RESEARCH LABORATORIES

OFFICE OF AEROSPACE RESEARCH

UNITED STATES AIR FORCE

WRIGHT-PATTERSON AIR FORCE BASE, OHIO 
This is a final report prepared by the Graduate Aeronautical Laboratories of the California Institute of Technology (GALCIT) for Aerospace Research Laboratories, Office of Aerospace Research, United States Air Force. The work was performed under Contract No. AF 33(615)-2217, Task 706302-N-3 of Project No. 7063. This report includes both analytical and experimental results obtained between 15 October 1964, and 15 November 1965, under the cognizance of the late Mr. Charles A. Davies, ARL Project Scientist.

It is appropriate to acknowledge the help of Mr. William Rae, lab technician and Marvin Jessey, electronics lab supervisor, for help in design, preparation and conduction of laboratory experiments. Furthermore, it is a pleasure to express appreciation to Mrs. Sally Richards and Mrs. Elizabeth Fox, who typed the manuscripts.

The notes and data for this report are recorded in GALCIT File No. SM 65-25. This report is distributed to the Chemical Propulsion Mailing List of 1965. 


\section{ABSTRACT}

The investigation of fracture of polymeric materials in hydrostatic tensile fields constitutes an avenue of approach to the study of fracture in more general three-dimensional environments. The advantages created by the symmetry of the stress field are considerable and, in one of the cases studied, facilitates a theoretical treatment that includes large deformations, which are characteristic of this class of materials.

The analysis is developed through the concept of fracture originating from a flaw, which in this instance is taken to be a spherical cavity. Through the application of energy principles, a theoretical prediction of ultimate strength is made for hydrostatic tensile fields.

Experiments were conducted to demonstrate the existence of such flaws and to evaluate the theory. Results of the tests on specimens containing both residual flaws and artificially inserted ones indicate a fundamental difference in behavior as contrasted with cracks.

An explanation is given linking experimental results and theoretical predictions. It is based on the concept that a flaw "grows"' in the material under load using the cavity as a nucleating point. Upon this hypothesis is built a theory of rupture in which planar cracks grow radially from the center of the cavity in the form of Saturn-ring cracks. 
TABLE OF CONTENTS

PROMINENCE OF HYDROSTATIC FIELDS

Hydrostatic Tension in Liquids

Hydrostatic Tension in Metals

Hydrostatic Tension in Polymers

Related Work

Previous Work in Hydrostatic Tension CHIP SPECIMEN

RELATED SOLUTIONS

APPROXIMATE SOLUTION

Simplification of the Stress Expression

Displacement Expression

Limit-Check of the Solution

Apparent Modulus

End Effect Parameter

The Effect of Corner Stress Singularities

COMPARISON OF RESULTS WITH OTHER SOLUTIONS

Finite Difference

Potential Energy Analysis

POKER - CHIP SPECIMEN SUBJECTED TO COMBINED TRIAXIAL LOADS 


$$
-\mathrm{v}-
$$

TABLE OF CONTENTS (Cont'd)

PART

TITLE

PAGE

Stress Analysis of Combined Torsion and

Extension

$\begin{array}{ll}\text { Principal Stresses } & 47\end{array}$

$\begin{array}{ll}\text { Observations } & 48\end{array}$

$\begin{array}{ll}\text { SUMMARY } & 54\end{array}$

III EXPERIMENTAL ANALYSIS IN HYDROSTATIC 56 TENSION

MATERIAL DESCRIPTION 56

Prepolymer Formation $\quad 56$

Chain Extension $\quad 57$

Curing or Crosslinking 58

MATERIAL FABRICATION 59

MATERIAL CHARACTERIZATION 63

$\begin{array}{ll}\text { Bulk Properties } & 68\end{array}$

$\begin{array}{ll}\text { Finite Strain Characterization } & 68\end{array}$

EXPERIMENTAL APPARATUS FOR THE 73 POKER - CHIP TEST

$\begin{array}{ll}\text { Bonding Procedure } & 75\end{array}$

$\begin{array}{ll}\text { Optics } & 77\end{array}$

$\begin{array}{ll}\text { Strain Measurements } & 78\end{array}$

$\begin{array}{ll}\text { EXPERIMENTAL RESULTS } & 79\end{array}$

Stress-Axis Theorem 82

Description of Fracture $\quad 84$

$\begin{array}{ll}\text { Fracture Surface } & 87\end{array}$

$\begin{array}{ll}\text { Fracture Propagation } & 90\end{array}$ 
TABLE OF CONTENTS (Cont'd)

ENERGY CRITERIA FOR ELASTIC FRACTURE 97

Conservation of Energy

Differential Form

ENERGY FUNCTIONALS

Extended Complementary Energy

100

STABILITY

101

Buckling Analogy

102

ENER GY FRACTURE ANALYSIS -

104 INF INITESIMAL THEORY

Critical Pressure 105

Flaw Size Dependency

106

Mode of Propagation 107

ENERGY FRACTURE ANALYSIS - EFFECT 107 OF FINITE DEFOR MATION

Finite Strain Effects

CRITICAL FRACTURE POINT

Strain Energy

Potential of Surface Forces

Surface Energy

CRITICALITY EQUATION

Comparative Results 
-vii-

TABLE OF CONTENTS (Cont'd)

PART

TITLE

PAGE

SATURN-RING CRACK

123

Strain Energy Function

125

Criticality Condition

130

Physical Interpretation

132

Limit Cases

136

Numerical Correlation

138

RELATED GEOMETRIES

141

Experimental Results

142

Character of $\mathrm{c}^{*}$

145

SUMMARY

146

VI CONCLUSION

147

REFERENCES

150 
$\sqrt{2}+x^{2}$ 


\section{CHAPTER I}

\section{INTRODUCTION}

PROMINENCE OF HYDROSTATIC FIELDS

Hydrostatic tension (HT) and hydrostatic compression (HC) constitute stress states of a very special class, possessing unique characteristics of symmetry. Probably the greatest amount of effort, and certainly the greatest number of results, have come from investigations with HC, as opposed to HT, where the outstanding work of Bridgman $(1,2)$ has received wide attention. Although his work has certainly gone well beyond simple HC, he has done considerable testing directly with it.

The relationship of $\mathrm{HT}$ and $\mathrm{HC}$ to the general three-dimensional stress state is conveniently displayed through a plot in principal stress space as shown in Figure 1. Here the effects of the symmetry are clearly borne out with the location of HT, or equal triaxial tension, being along a line that bisects the solid angle formed by the three principal axes and extending infinitely in the positive direction. The HC counterpart is the extension of this locus in the negative direction.

To further illustrate the role of symmetry in fracture it is possible to construct a failure surface in principal stress space, defined by

$$
f\left(\sigma_{1}, \sigma_{2}, \sigma_{3}\right)=0
$$

which is a locus of points forming a limiting surface of rupture; i.e., points whose principal stress coordinates lie within the 


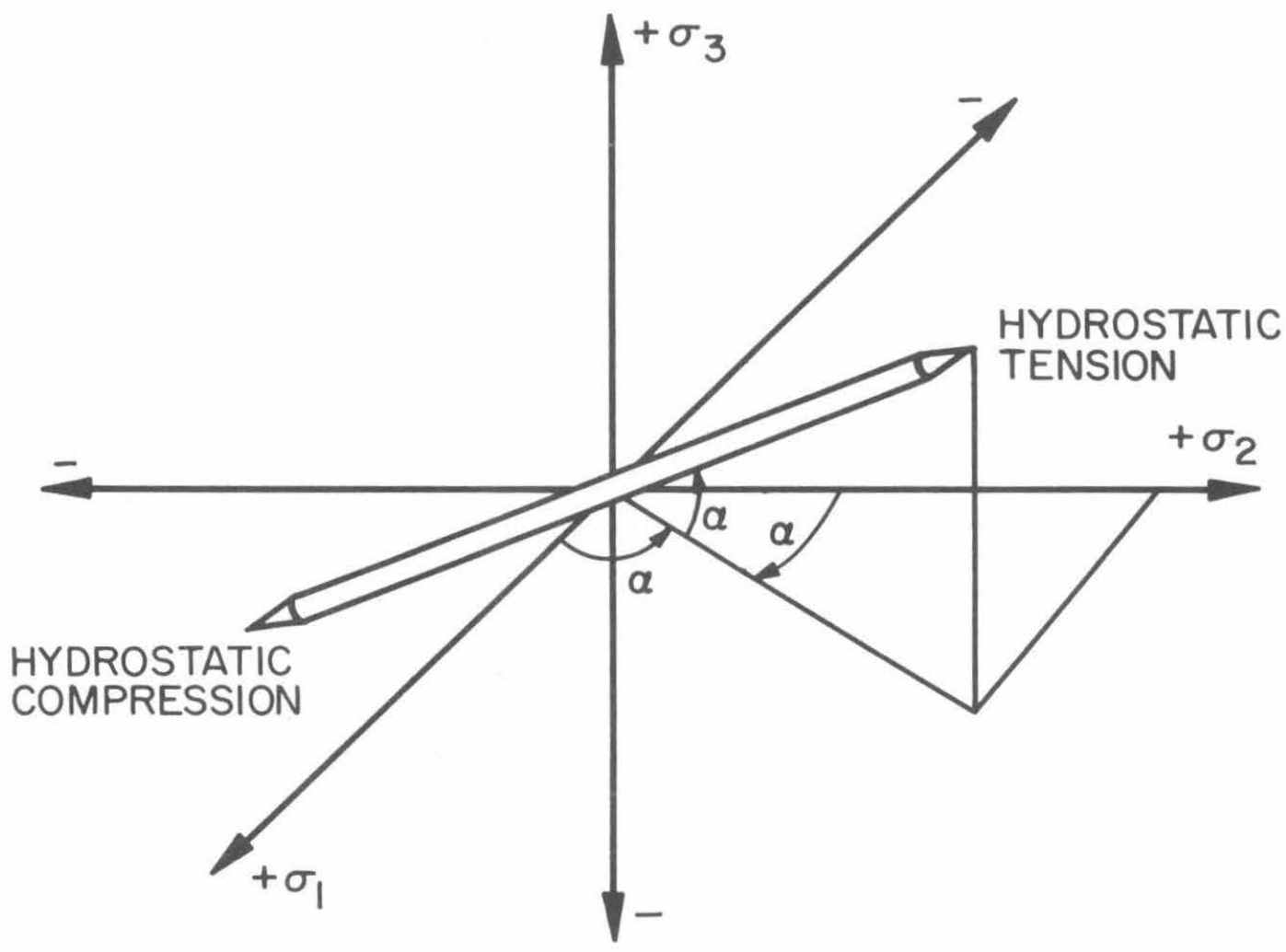

Figure 1. Locus of Hydrostatic Tension and Hydrostatic Compression in Principal Stress Space. 
surface will not fail and those lying on or above the surface will produce failure. Blatz (3) has shown that in general failure, either from actual rupture or excessive deformation, can be broken down into a dilatational contribution and a distortional contribution. He has plotted the pure failure modes of dilatation and distortion in principal stress space in Figure 2. The stress quality in all octants may be denoted as follows:

\begin{tabular}{|c|c|c|c|c|}
\hline Octant & $\sigma_{1}$ & $\sigma_{2}$ & $\sigma_{3}$ & $\begin{array}{c}\text { Number of } \\
\text { Positive Stresses } \\
\end{array}$ \\
\hline I & + & + & + & 3 \\
\hline II & + & + & - & 2 \\
\hline III & + & - & + & 2 \\
\hline IV & + & - & - & 1 \\
\hline V & - & + & - & 2 \\
\hline VI & - & + & - & 1 \\
\hline VII & - & - & + & 1 \\
\hline VIII & - & - & - & 0 \\
\hline
\end{tabular}

By virtue of equivalence of the three principal axes, it is noted that there are only four categories of octants characterized by the number of stresses of the same sign. Thus octants II, III and V are similar, and octants IV, VI, and VII are similar. This means that, for an isotropic material, only four octants need to be tested. It also means that since the axes of principal stress must be invariant 


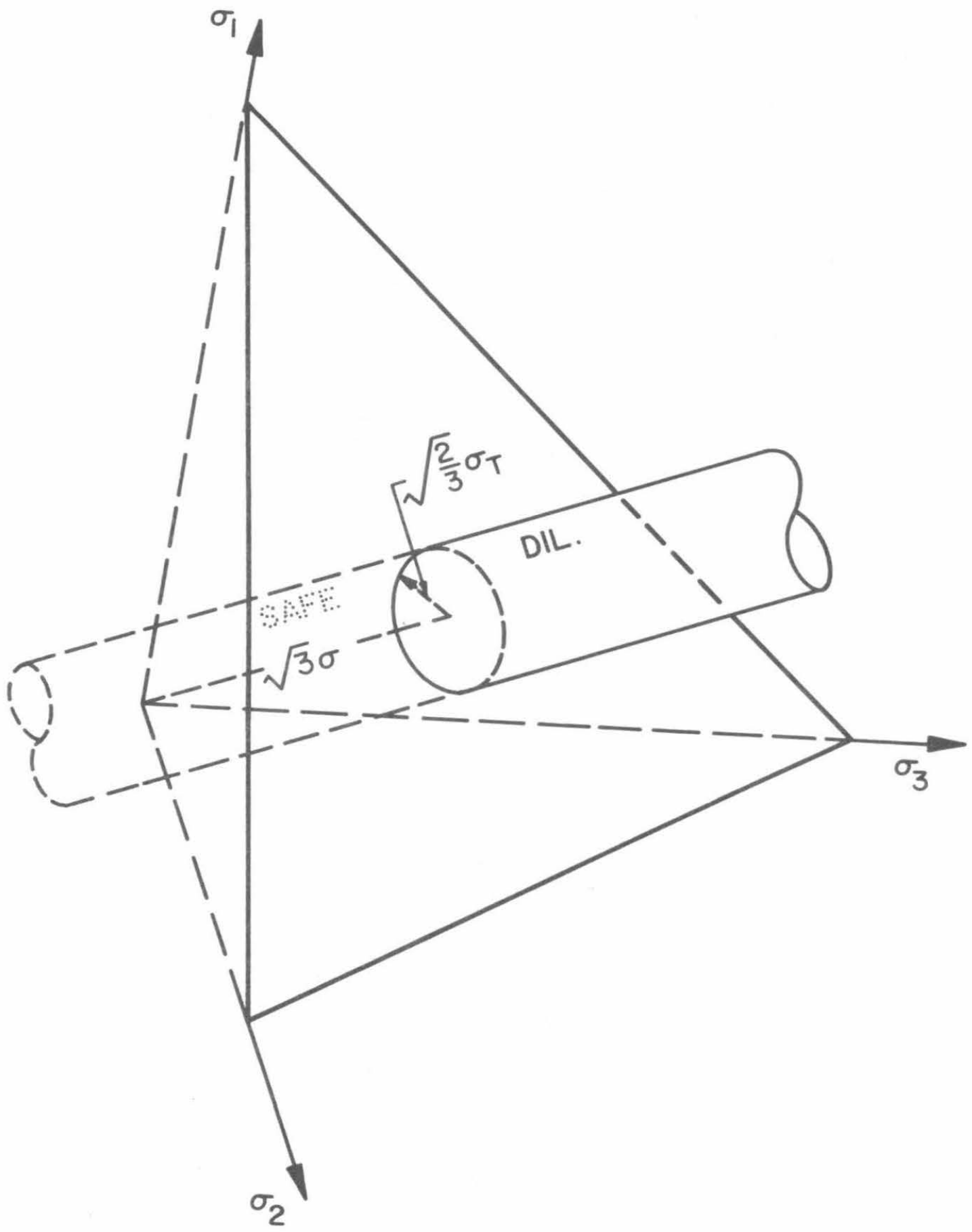

Figure 2. Fail-Safe Surface Based on the Strain-Energy Criterion
in Normal Stress Space. 
to the group of rotations in the body, the hydrostatic line becomes an axis of symmetry and consequently fracture in HT and HC become extremums; i.e., they are limit points on the failure surface.

Now although there are many obvious similarities between $\mathrm{HT}$ and $\mathrm{HC}$, there is a great deal of difference in the manner in which materials respond to these two environments fracturewise. The theory cannot demonstrate that there will be a difference in the actual configuration of the failure surface; however it has been found through experiment that there are significant differences. Bridgman (1) has shown that in combined stress states involving high levels of hydrostatic pressure, none of the standard failure criteria of maximum principal stress, maximum principal strain, etc. postulated from tensile results are accurate. He has investigated many stress states that cover several of the octants in principal stress space and has found large alterations in the levels of ultimate strains and ultimate stresses in these other octants when compared to the +++ octant. He has also discussed (2) the fact that it is necessary before rupture can occur to have what he terms an energy release mechanism, or more simply, a place for the material to go so that energy can be used to create new surface. Reflection upon this point leads to the conclusion that in pure hydrostatic compression fracture could never occur and the ultimate strength would be infinite. However, slight perturbations from this field would provide enough anti-symmetry to allow fracture to occur at realistic levels. Therefore in hydrostatic compression the failure surface possesses a cusp at infinity, which would be in strong contrast to the same situation in tension 
where there is an energy release mechanism, and fracture can occur at finite values. It then follows that $\mathrm{HT}$ and $\mathrm{HC}$ produce quite a different effect upon materials, and the resulting behavior in each case is not the inverse of the other.

One additional point that needs further emphasis is the mathematical simplicity that arises as a consequence of the symmetry in the problem. The number of existing solutions to three-dimensional elasticity problems is limited, but in this instance the theoretical analysis accompanying the experiment is not only possible but reasonably simple, even in the case of finite deformation theory. (4) It makes a solution possible where it otherwise may be intractable. All of these factors combine to make HT and HC fields of considerable interest, as well as of considerable value.

Hydrostatic Tension in Liquids

Although HC lends itself well to experimentation, states of HT are not so readily generated in the laboratory. One exception to this is in the case of liquids where HT can readily be created, and as a consequence, fracture of several liquids has been investigated. Studies of this type not only contribute to the fundamental knowledge of physics, but are of considerable engineering interest as they relate to the phenomena of cavitation. Fisher (6) has applied an energy balance to the growth of a spherical cavity in a liquid, and from this has been able to derive an expression defining the critical pressure at which the bubble will grow. 


$$
\mathrm{p}_{\mathrm{c}}=\frac{2 \gamma}{\mathrm{r}}-\mathrm{p}_{\mathrm{v}}
$$

where $\mathrm{p}_{\mathrm{C}}=$ critical pressure

$$
\begin{aligned}
\mathrm{\gamma} & =\text { surface tension } \\
\mathrm{r} & =\text { radius of cavity } \\
\mathrm{p}_{\mathrm{V}} & =\text { vapor pressure (usually neglected) }
\end{aligned}
$$

Irwin (7) has extended the analysis of Fisher and favorably compared his theory with data on the fracture of liquids obtained from several other investigators. A large number of experimental uncertainties, very difficult to control, made the correlation somewhat fortuitous, and thus led Irwin to conclude that the theoretical strength calculations for pure liquids were of doubtful practical utility. Nevertheless, as he states, the degree of completeness permitted in the theoretical considerations, primarily due to the symmetry involved, make the pure liquid tensile strength analysis of importance. Furthermore, it can act as a limit case for the more general viscoelastic material, which we will refer to in a later chapter.

\section{Hydrostatic Tension in Metals}

Fracture studies for general combined stress states have been pursued for metals quite arduously. (8) With complex testing equipment capable of applying fluid pressure as well as tensile shear and bending loads, it has been possible to study metallic fracture under a wide range of loading conditions, but not HT. Nadai (9) has traced some of the attempts to create HT in metals. Two of the methods employed, which are of a similar nature, are thermal stresses and 
grain transformations. Here attempts are made to produce a state in the body such that one portion pulls on another portion, putting it in $H T$, either by thermal gradient or by volume change due to phase transformation. Although some success has been realized in producing the desired condition, it has never been possible to obtain any quantitative measurements from such tests. Another popular method has been the use of a circumferential notch on a circular cylindrical bar. It was believed that the stress state at the base of a sharp hyperbolic notch was hydrostatic when the bar was under axial tension. However, Neuber (10) in his treatise on notch stresses demonstrated that this was not the case and that near the surface of the notch the stress ratios were actually $1: 1: 3$, with the largest being in the axial direction. Unfortunately the test has not been useful for other combined tensile stress states in the +++ octant due to the large gradients of stress in the neighborhood of the notch, which is the region of interest. Still another attempt was made by Lehrer and Schwalzbart (11) as they bonded a thin sheet of brass between two plates of steel and pulled the plates in tension perpendicular to the large, flat face. This test has promise but primarily for materials that are nearly incompressible. This will be demonstrated in a later analysis.

So in metals it still remains to find a good hydrostatic tensile test; although almost any other combination of stress can be imposed. It is interesting that in spite of this missing piece of information McAdam, (12) from other experiments, postulated that HT would be an extremum point on a convex fracture surface; i.e., this stress 
state would represent the maximum in ultimate strength that could be enjoyed by a brittle metal in tension. It is also interesting to note that this has subsequently turned out to be the case in other engineering materials, such as polymers, where it is possible to produce HT in the laboratory.

Hydrostatic Tension in Polymers

Polymeric materials are fundamentally different in their basic structure and in their behavior. $(13,14,15)$ Their difference is so pronounced that special methods of stress analysis, upon which fracture analysis is built, have had to be developed. The state of this art has recently been reviewed by Williams. (16) Differences in the basic structure produce differences in their fracture behavior, which has been reviewed first by Bueche and Berry, (17) and subsequently by Williams. (18) Widespread interest in the fracture properties of these materials has arisen through a vastly expanding usage of polymers in engineering applications where structural integrity is an item of concern. One primary example, which attracts the interest of Aeronautical Engineers, is the structural integrity of solid propellant rocket grains. (19) In this instance, the solid propellant fuel constitutes an integral part of the structure; thereby requiring analysis of its material integrity like any other structural component. However, the constitution of these materials is very complex. It consists of a binder material, which is an amorphous elastomer, impregnated with a high volume percentage of solid oxidizer particles such as ammonium perchlorate. This system is neither 
homogeneous nor isotropic, and it is innately very complicated in its mechanical behavior. It therefore is reasonable to seek a simplified approach to the problem with the aim of discovering some of the fundamentals of the behavior of the separate components; i.e., to investigate the fracture properties of the amorphous rubber binder as a first approach to an investigation of the filled system. An examination of fracture in amorphous rubber also has its own inherent interest as it would apply to other engineering applications where there is no filler involved; thus the incentive for the investigation of fracture is two-fold: (i) the attempt to study failure in solid propellant materials for their own sake, and (ii) to discover general principles that can be applied directly to the fracture analysis of engineering components where the amorphous polymer alone is the structural material.

Related Work. In order to place the HT work in proper perspective, reference will be made to related work in other stress states. Most of the effort expended on unfilled elastomers has thus far been applied to the case of uniaxial tension. Certainly this is the logical starting point, for it keeps complication to a minimum, so that experimental results are not obscured by extraneous influences. However, even in this simple case much work has been required to uncover and define basic behavior in terms of mechanical properties. A comprehensive review of the uniaxial work has currently been given by Landel and Fedors, (20) which devotes some attention to the elusive problem of fracture properties under general loading 
conditions. This is the area in which there is still much to be done even in the uniaxial state, for the concepts of fatigue and cumulative damage are not only unsettled, but investigations are still in their infancy even though preliminary information is now coming forth as evidenced by the work of Knauss and Betz. (21)

Concurrently there has been a similar, but smaller, corps of investigators working with multiaxial polymer fracture. Two biaxial tests have been used, one referred to as the strip biaxial which has probably received the most attention is shown in Figure 3 and the other is that of the equal biaxial test, which can be conducted by inflating a membrane (Figure 4) or in some instances special fixtures have been successful. (See Ko (22).) These tests are quite tedious. Furthermore it is difficult to force fracture to occur away from the grips, and they require considerable care in the preparation of the specimen to yield a cross-section that will produce the desired stress field; consequently a limited amount of results is available for these geometries. One extensive work using several stress fields in uniaxial, biaxial, and triaxial tension to construct failure surfaces has been completed by Ko. (22)

Previous Work in Hydrostatic Tension. There has been even less work done in the area of triaxial fracture. One of the first efforts in this direction was made by Gent and Lindley, $(23,24)$ who performed tests in HT and HC. They were attracted to an unusual test by which they produced these fields following work reported by Yerzley (25) on the bond integrity between rubbery materials. In 


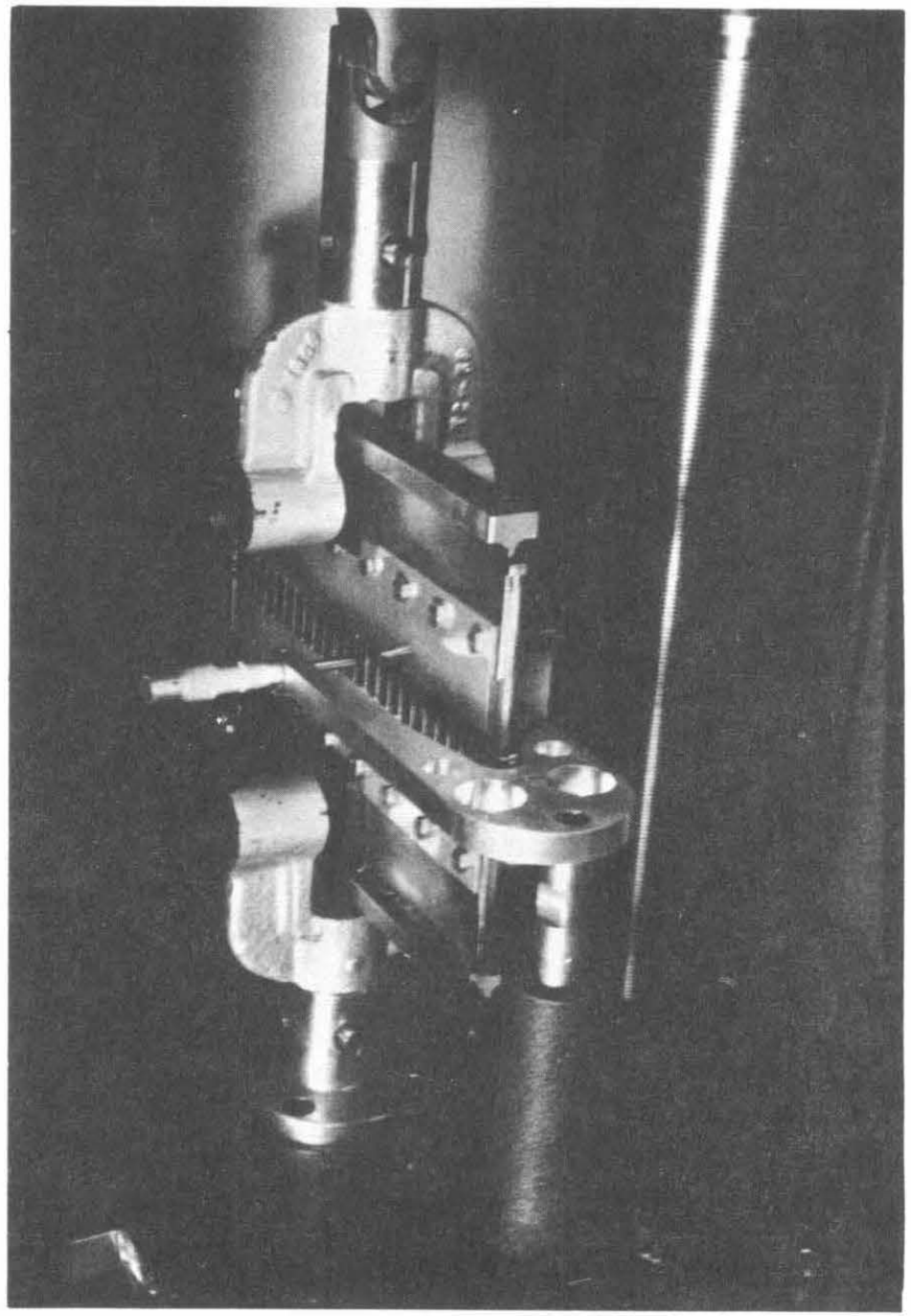

Figure 3. Strip-Biaxial Tension. 


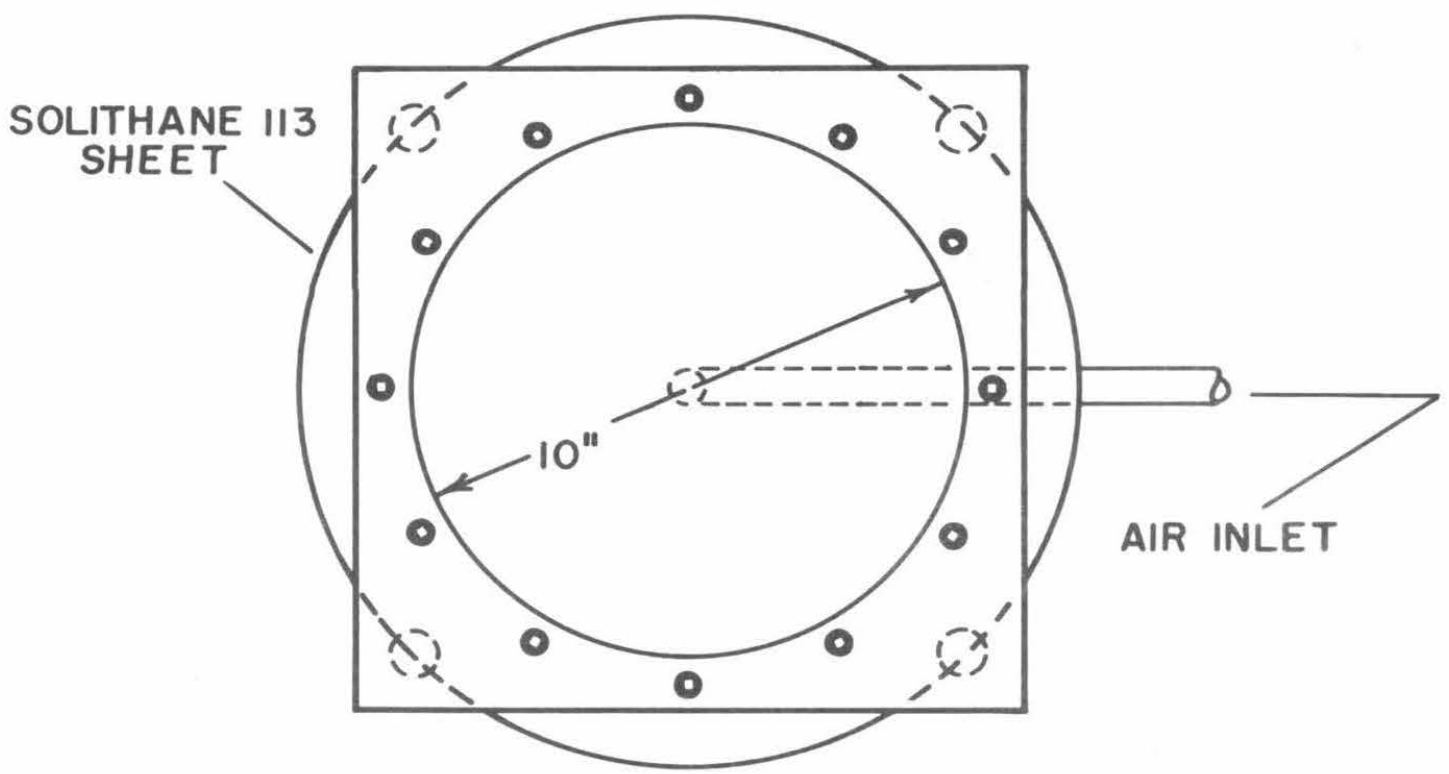

SOLITHANE II3

SHEE T - (INFLATED )

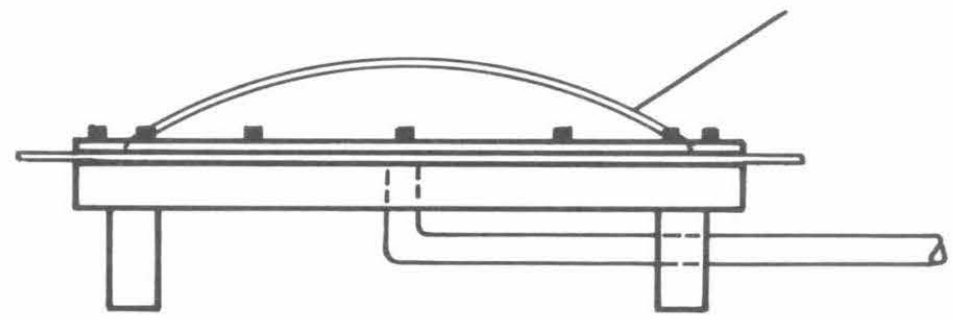

Figure 4. Equal Biaxial Membrane Test. 
Yerzley's search for an ASTM standard, he glued a single thin rectangular block of rubber between two similar metal blocks and pulled them apart to test the strength of the interface bonds. In the course of the experiments, he noted a rather peculiar type of fracture in the rubbery specimen and discussed it briefly. Twenty years later Gent and Lindley pursued this test by manufacturing some small circular disks from a carbon black filled, natural rubber and pulled them by means of two, rigid, steel plates. With the thin disk of soft specimen material glued and sandwiched between the stiffer grips, it will be restrained from contracting laterally as the entire assembly is extended along its axis perpendicular to the face of the disk. This creates the triaxial stress field. The amount of restraint is a function of the aspect ratio (diameter to thickness) of the specimen, but an elementary analysis can be made by assuming the disk to be infinitely thin such that the external radius is sufficiently far from the center to assume that the only non-zero displacement, $\underline{w}$, is in the $\mathrm{x}_{3}$ direction. With this configuration the boundary conditions become $\mathrm{u}=\mathrm{v}=0$ from which $\epsilon_{\mathrm{r}}=\epsilon_{\theta}=0$. The stress field then becomes

$$
\sigma_{\mathrm{r}}=\sigma_{\theta}=\frac{v}{1-v} \sigma_{\mathrm{z}}
$$

where use has been made of the axial strain

$$
\varepsilon_{\mathrm{z}}=\frac{\sigma_{\mathrm{z}}}{\mathrm{E}} \frac{(1-2 v)(1+v)}{(1-v)}
$$

so that the apparent axial modulus becomes 


$$
\mathrm{E}_{\mathrm{A}}=\mathrm{E}\left[\frac{1-v}{(1-2 v)(1+v)}\right]
$$

where it may be noted that for rubbery materials which are characteristically incompressible, i.e., $v=1 / 2$, the triaxial tensile stress state approaches hydrostatic with the consequent infinite apparent axial stiffness. Gent and Lindley's initial experimental work demonstrated an internal fracture in the rubber which varied with thickness, modulus and tensile strength, and they devoted their attention to documenting and explaining this variation. After completing a preliminary probe into this interesting mode of fracture, they extended their work to compression using carbon black filled rubber specimens. Emphasis was placed on defining the load-deflection relation and obtaining a definition of the stress field in the specimen.

\section{METHOD OF APPROACH}

The work of Gent and Lindley will be used as a point of departure for the work to be reported. The first item to receive attention will be a detailed stress analysis of the test specimen to provide a means of local examination of the experimental results. This will be coupled with experimental work made on a modified test apparatus, which permits a more detailed study of the fracture process. Interpretation of these results and analytical extensions thereof will then be made on the basis of a flaw hypothesis. A word of justification for this assumption is in order.

Many analytical and experimental techniques currently applied to polymers were carried over from metal fracture. 
Although several have been found to apply directly, as yet no universal approach has been discovered. However, in specific instances, particular approaches have been fruitful. For instance, through laboratory experience it has been found that many polymers are very notch sensitive; i.e., their properties are strongly controlled by imperfections on the surface as well as in the interior of the body. Such behavior suggests that an investigation of the fracture phenomenon in these materials may appropriately be made by means of a flaw hypothesis. This method can be employed on either the molecular or the continuum level. Physical chemists have studied the effects of flaws in the chain structure itself, and worked up by statistical means, through groups of chains, to the continuous specimen, where correlations can be made between specimen load and localized stress at the molecular flaw. A consideration of the bond energies then leads to a prediction of fracture. Early ideas of this type were put forth by Houwink (26) and later expanded by investigators such as Flory, (27) who was an advocate of molecular flaws due to dangling chain ends. Currently this approach is yielding results due to improved mathematical techniques, including the recent work of Blatz (28) and Knauss (29).

An alternate approach is to consider the material initially as a continuum and then represent the flaws as discontinuities in that continuum. Through an analysis of a typical flaw, which in one instance is a spherical cavity taken to be independent of all other flaws in the material, the local conditions of stress, strain, and energy, can be computed and fracture predicted through the 
application of an energy criterion. (30) This will be done for two different modes of propagation of the fracture surface, and subsequently a comparison of the two leads to new insight into the behavior of holes and cavities. One further point should be noted before proceeding. Amorphous elastomers characteristically are viscoelastic (31) and exhibit large deformations in fracture. (32) These characteristics complicate the analysis considerably, especially as the theory of finite viscoelasticity has not yet progressed to the point that it is a practical tool for analysis. * For this reason the work referred to herein, as well as this entire effort, is predominantly performed with the classical tools of infinitesimal elasticity (and in some cases infinitesimal viscoelasticity) and should be interpreted as an exploration of the broad concepts of polymeric fracture in $\mathrm{HT}$ rather than a final definitive treatise of the subject.

* Schapery (33) has just completed a report that promises to help rectify this situation and make finite viscoelasticity a bit more manageable for engineering analysis. 


\section{CHAPTER II}

\section{THEORETICAL ANALYSIS OF THE POKER -CHIP SPECIMEN}

The mathematical model of the poker-chip configuration, shown in Figure 5, leads to a mixed boundary value problem that is almost analytically intractable from the standpoint of classical elastic theory. Like many elastic problems involving finite bodies with discontinuous boundary conditions, the poker-chip configuration presents many mathematical difficulties if an exact, closed-form solution is sought. However, for such problems series solutions are possible and several have been used.

\section{RELATED SOLUTIONS}

One of the first theoretical analyses relating to this problem was published by Pickett. (34) In his analysis of cylinders, he employed a Fourier series expansion, which resulted in the final solution being expressed in terms of a doubly infinite series. This form is awkward for our purpose, where the results are involved in a subsequent analysis of fracture, especially where convergence of the solution is slow. This is emphasized near the corners, where it should be noted that the problem of convergence is basic due to the peculiar geometrical effects present there. There is a discontinuity in material, as well as discontinuities from stress to displacement boundary conditions, and this may lead to mathematically infinite stresses. (35) When the stress singularity does occur, and such singular behavior has not been explicitly built into the form of the formal representation of the 


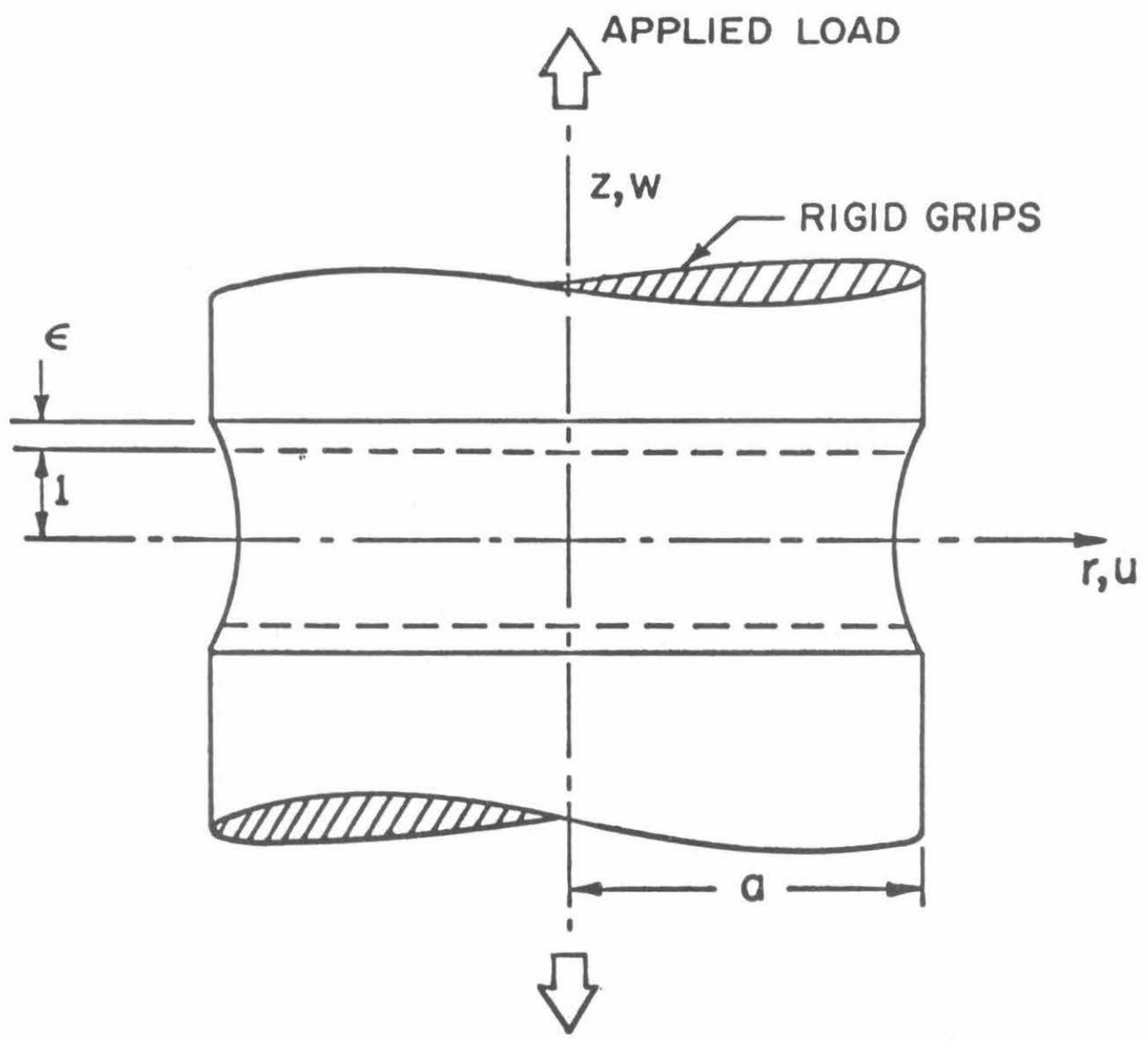

Figure 5. Triaxial "Poker-Chip" Test and the Coordinate System Used in the Stresa Analysis. 
solution, convergence and rate of convergence difficulties are to be expected.

Gent and Lindley (23) began by solving an analogous problem of an infinite slab, using intuitive assumptions based on incompressible material behavior and proposed a stress analysis, which can be shown equivalent to minimizing the potential energy. They then extended this line of reasoning to the circular disk, and the apparent modulus ${ }^{*}$ deduced from this analysis was compared to a large amount of experimental data. Qualitatively there was good agreement, although quantitative predictions with the theory were good only for a very small range of aspect ratios. Furthermore, since the apparent modulus is essentially an average property, the corresponding internal stresses needed for failure analysis could be significantly different than the average value.

Energy methods have also been used. One of the first was a complementary energy formulation in terms of the stresses used by Williams, Blatz, and Schapery. (19) In cylindrical coordinates the proposed stress representations were

$$
\begin{aligned}
& \sigma_{r}=\frac{2 v}{1-v} A\left(1-r^{n}\right) \cosh \sqrt{\frac{2 v}{1-v}} z \\
& \sigma_{\theta}=\frac{2 v}{1-v} \mathrm{~A}\left[1-(\mathrm{n}+1) \mathrm{r}^{\mathrm{n}}+\mathrm{r}^{\mathrm{p}-1}-\mathrm{r}^{2}\right] \cosh \sqrt{\frac{2 v}{1-v}} \mathrm{z} \\
& \sigma_{\mathrm{z}}=\sigma_{\mathrm{O}}+\left[1-\frac{\mathrm{p}+1}{2} \mathrm{r}^{\mathrm{p}-1}\right]\left[2 \mathrm{~A} \cosh \sqrt{\frac{2 \nu}{1-\nu}} \mathrm{z}-\sigma_{\mathrm{O}}\right]
\end{aligned}
$$

* Following Gent and Lindley, the apparent modulus is defined as the average axial stress divided by the applied axial strain. 


$$
\tau_{\mathrm{rz}}=-\mathrm{A}\left[\mathrm{r}-\mathrm{r}^{\mathrm{p}}\right] \sqrt{\frac{2 \nu}{1-v}} \sinh \sqrt{\frac{2 v}{1-v}} \mathrm{z}
$$

where the four constants $\sigma_{0}, \mathrm{p}, \mathrm{n}$, and $\mathrm{A}$ were to be determined by minimizing the complementary energy. On the other hand, a potential energy formulation in terms of displacements has been proposed by Francis and Cantey (36) in which the radial and axial displacements, minimizing the energy, were found to be of the form ${ }^{*}$

$$
\begin{aligned}
& \mathrm{u}=\left[1-\left(\frac{\mathrm{z}}{\mathrm{h}}\right)^{2}\right]\left[\mathrm{k}_{0} \mathrm{~J}_{1}\left(\gamma_{2}, \mathrm{r}\right)\right] \\
& \mathrm{w}=\left(\frac{\mathrm{z}}{\mathrm{h}}\right)\left[1-\left(\frac{\mathrm{z}}{\mathrm{h}}\right)^{2}\right]\left[\mathrm{k}_{1} \mathrm{~J}_{0}\left(\gamma_{1}, \mathrm{r}\right)+\mathrm{k}_{2} \mathrm{~J}_{0}\left(\gamma_{2}, \mathrm{r}\right)\right]
\end{aligned}
$$

In both of these cases, however, the algebraic complexities involved in the computation were found to be rather formidable, particularly when parametric variations of aspect ratio and material constants were desired. Furthermore, as is fundamental with these particular energy solutions, the complementary principle for stress approximation usually yields poor displacements and, vice versa, the potential energy principle for displacements does not satisfy stress equilibrium. It will be shown later however that a combination of these methods can yield good engineering accuracy.

Still another solution came as a result of a preliminary study for the poker-chip specimen in the form of a potential energy solution for a two-dimensional slab. (37) It made use of the careful edge displacement measurements of Gent (38) in which he determined the

Note that $w=0$ at $z=h$. This solution is to be superimposed with the constant strain solution. 
transverse displacements to be mainly parabolic functions of the longitudinal (axial) coordinate. Results of the slab analysis furnished an increased understanding of the complete stress distribution, including the extent of the boundary influence on the internal stresses and the fact that the axial displacement of the slab was virtually constant throughout. Building upon this foundation it was possible to compute two approximate solutions for the disk. The first involves a technique of averaging the stresses through the thickness of the specimen and satisfying the equilibrium equations on an average basis. The other employs the variational approach for the minimization of the potential energy. Both use assumed functional forms for the displacements, which are guided by the slab analysis, and the two methods bear a strong similarity throughout. This will be demonstrated in detail later as both solutions are discussed.

Finally, numerical solutions to the problem have been obtained by Messner, (39) and Brisbane. (40) In Messner's solution, for instance, a finite difference technique has been used, and the grid size has been progressively reduced until two subsequent sizes produce no appreciable change in the stress state. The calculated stress distribution will be presented graphically later to act on a basis of comparison for the accuracy of the approximate analytical solution. The convergence at the corners has been found to be extremely slow, and this is again due to the presence of stress singularities at these points. This type of computer program offers great practical advantages, since a 
solution can be obtained to any desired accuracy in a short time. However it has the disadvantage that in order to perform a parametric study, a separate calculation has to be performed for each configuration.

\section{APPROXIMATE SOLUTION}

This section describes an approximate method for analyzing a thin, circular disk developed by Lindsey, Schapery, Williams and Zak. (41) As mentioned previously it uses an extension of the solution for a slab in plane strain. (37) One of the primary advantages of this solution is that the incompressibility assumption made by Gent and Lindley (23) does not have to be invoked so that the resulting solution is applicable over a range of material properties. Figure 5 shows a circular disk of radius a with its axis in the $\mathrm{z}$-direction, and the faces $\mathrm{z}= \pm 1$ bonded to rigid plates. We assume that the disk is loaded by increasing the thickness by $2 \epsilon$ and proceed to select two displacement functions, which satisfy the boundary conditions on that part of the boundary where displacements are prescribed. Note that the third (circumferential) displacement, $v$, is identically zero by reasons of symmetry. Such functions would also be admissible functions for use in the Theorem of Minimum Potential Energy, although it should be recalled that the resultant minimization yields a result, in this case it will turn out to be the function $g(x)$, such that the equations of equilibrium are not satisfied unless the solution is actually exact. The radial displacement function is known to be essentially 
parabolic from the edge measurements by Gent. (38) The longitudinal displacement was found by Schapery and Williams (37) to be directly proportional to its distance from the horizontal plane of symmetry over most of the disk. Consequently the displacement functions take the form

$$
\begin{aligned}
& \mathrm{u}=-\left(1-\mathrm{z}^{2}\right) \mathrm{g}(\mathrm{r}) \\
& \mathrm{w}=\epsilon \mathrm{z}
\end{aligned}
$$

in the radial and thickness directions respectively. Note that the displacement boundary conditions are satisfied at the surfaces $z= \pm 1$, and that $g(r)$ is presently an unprescribed function of the radius. The strains corresponding to these displacements are easily found to be

$$
\begin{aligned}
& \epsilon_{r}=\frac{\partial u}{\partial r}=-g^{\prime}\left(1-z^{2}\right) \\
& \epsilon_{\theta}=\frac{u}{r}=-\frac{g}{r}\left(1-z^{2}\right) \\
& \epsilon_{z}=\frac{\partial w}{\partial z}=\epsilon \\
& \gamma_{r z}=\frac{\partial u}{\partial z}+\frac{\partial w}{\partial r}=2 g z
\end{aligned}
$$

from which the $\mathrm{z}$-averaged stresses are found as

$$
\begin{aligned}
& \bar{\sigma}_{\mathrm{r}} \equiv \frac{1}{2} \int_{-1}^{1} \sigma_{\mathrm{r}} \mathrm{dz}=\lambda\left(\epsilon-\frac{2}{3} \mathrm{~g}^{\prime}-\frac{2}{3} \frac{\mathrm{g}}{\mathrm{r}}\right)-\frac{4}{3} \mu \mathrm{g}^{\prime} \\
& \bar{\sigma}_{\theta} \equiv \frac{1}{2} \int_{-1}^{1} \sigma_{\theta} \mathrm{dz}=\lambda\left(\epsilon-\frac{2}{3} \mathrm{~g}^{\prime}-\frac{2}{3} \frac{\mathrm{g}}{\mathrm{r}}\right)-\frac{4}{3} \mu \frac{\mathrm{g}}{\mathrm{r}} \\
& \bar{\sigma}_{\mathrm{z}} \equiv \frac{1}{2} \int_{-1}^{1} \sigma_{\mathrm{z}} \mathrm{dz}=\lambda\left(\epsilon-\frac{2}{3} \mathrm{~g}^{\prime}-\frac{2}{3} \frac{\mathrm{g}}{\mathrm{r}}\right)+2 \mu \epsilon
\end{aligned}
$$




$$
\tau_{\mathrm{rz}}=2 \mu \mathrm{gz}
$$

where $\mathrm{g}^{\prime} \equiv \mathrm{dg} / \mathrm{dr}$.

The function $g$ is found from the condition that the $z$-integrated. equilibrium equation for the radial direction is to vanish, i.e.,

$$
\frac{1}{2} \int_{-1}^{1}\left[\frac{\partial{ }_{r}}{\partial r}+\frac{\partial \tau}{\partial z}+\frac{{ }^{\sigma}{ }_{r}-\sigma \theta}{r}\right] d z=0
$$

or

$$
\frac{\mathrm{d} \bar{\sigma}_{\mathrm{r}}}{\mathrm{dr}}+\left.\tau_{\mathrm{rz}}\right|_{\mathrm{z}=1}+\frac{\bar{\sigma}_{\mathrm{r}}-\bar{\sigma}_{\theta}}{\mathrm{r}}=0
$$

Also, note that because of symmetry the integrated equilibrium equation for the $\mathrm{z}$-direction is satisfied identically,

$$
\int_{-1}^{1}\left[\frac{\partial \tau}{\partial z}+\frac{\partial \sigma z}{\partial z}+\frac{{ }^{r} r z}{r}\right] d z=0
$$

Substitution of stresses (2.9) into equation (2.10) yields the differential equation for $g$, thus

$$
g^{\prime \prime}+\frac{g^{\prime}}{r}-\left(\frac{1}{r^{2}}+M\right) g=0
$$

where $\mathrm{M}$ is a constant made up of a composite of material properties.

$$
M \equiv \frac{3 \mu}{\lambda+2 \mu}=\frac{3}{2} \frac{(1-2 v)}{(1-v)}
$$

Equation (2.12) is a form of Bessel's Equation and yields modified Bessel functions as solutions. 


$$
g(r)=\operatorname{AI}_{1}(r \sqrt{M})+B K_{1}(r \sqrt{M})
$$

where $I_{1}(r \sqrt{M})$ and $K_{1}(r \sqrt{M})$ are modified Bessel functions of the first and second kind respectively. Since $K_{1}(0)$ is infinite, and we require the deformation to be finite at the origin, $B=0$. The constant $A$ is found by using the boundary condition that $\bar{\sigma}_{r}(a)=0$ from which it follows that

$$
A=\frac{3}{2} \frac{v \epsilon}{(1-v) \sqrt{M} I_{0}(a \sqrt{M})-(1-2 v) \frac{I_{1}(a \sqrt{M})}{a}}
$$

Substitution of the solutions (2.14) into stresses (2.9) using (2.15) yields the following stresses:

$$
\begin{aligned}
& \frac{\bar{\sigma}_{r}}{E \epsilon}=\left(\frac{3 v}{1+v}\right) \frac{K}{E}\left[1-\frac{I_{0}(r \sqrt{M})\left\{1-\frac{2}{3} M \frac{I_{1}(r \sqrt{M})}{r \sqrt{M} I_{0}(r \sqrt{M})}\right\}}{I_{0}(a \sqrt{M})\left\{1-\frac{2}{3} M \frac{I_{1}(a \sqrt{M})}{a \sqrt{M} I_{0}(a \sqrt{M})}\right\}}\right] \\
& \bar{\sigma}_{\theta}=\frac{\bar{\sigma}_{r}}{\bar{E} \epsilon}+\frac{1}{1+v}\left[\frac{I_{0}(r \sqrt{M})-\frac{2 I_{1}(r \sqrt{M})}{r \sqrt{M}}}{I_{0}(a \sqrt{M})+\left(\frac{1-2 v}{v}\right)\left\{I_{0}(a \sqrt{M})-\frac{I_{1}(a \sqrt{M})}{a \sqrt{M}}\right\}}\right] \\
& \bar{\sigma}_{z}=\frac{\bar{\sigma}_{r}}{E}+\frac{1}{1+v}\left[\begin{array}{c}
1+\frac{I_{0}(r \sqrt{M})}{I_{0}(a \sqrt{M})+\left(\frac{1-2 v}{v}\right)\left\{I_{0}(a \sqrt{M})-\frac{I_{1}(a \sqrt{M})}{a \sqrt{M}}\right.}
\end{array}\right]
\end{aligned}
$$




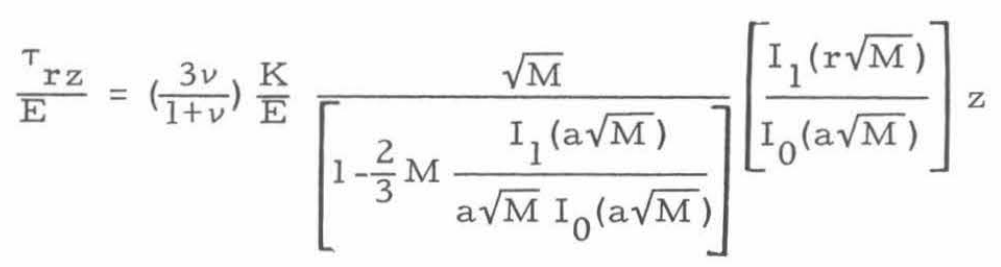

which, it may be found, approaches a state of triaxial, hydrostatic tension at the center in the case of an incompressible material. We expect the stresses $(2.16)$ to be good approximations for $0 \leqslant v \leqslant 1 / 2$ except for the singular stresses near the free-edge $r=a$.

Simplification of the Stress Expression. With this restriction on the radius, equations (2.16) can be very well approximated by the expressions:

$$
\begin{aligned}
& \frac{\bar{\sigma}_{\mathrm{r}}}{\mathrm{E} \epsilon}=\frac{\bar{\sigma}_{\theta}}{\mathrm{E} \epsilon} \cong \frac{3 v}{1+v}\left(\frac{\mathrm{K}}{\mathrm{E}}\right)\left[1-\frac{\mathrm{I}_{0}\left(\mathrm{r} \sqrt{\left.\frac{\mathrm{E}}{\mathrm{K}}\right)}\right.}{\mathrm{I}_{0}\left(\mathrm{a} \sqrt{\left.\frac{\mathrm{E}}{\mathrm{K}}\right)}\right.}\right] \\
& \frac{\sigma_{\mathrm{z}}}{\mathrm{E} \epsilon} \cong \frac{3 v}{1+v}\left(\frac{\mathrm{K}}{\mathrm{E}}\right)\left[1-\frac{\mathrm{I}_{0}\left(\mathrm{r} \sqrt{\frac{\mathrm{E}}{\mathrm{K}}}\right)}{\mathrm{I}_{0}\left(\mathrm{a} \sqrt{\frac{\mathrm{E}}{\mathrm{K}}}\right)}\right]+\frac{1}{1+v}\left[1+\frac{1}{2} \frac{I_{0}\left(\mathrm{r} \sqrt{\left.\frac{\mathrm{E}}{\mathrm{K}}\right)}\right.}{I_{0}\left(a \sqrt{\frac{\mathrm{E}}{\mathrm{K}}}\right)}\right] \\
& \frac{\tau_{\mathrm{rz}}}{\mathrm{E}} \cong \frac{3 v}{\mathrm{l}+v} \sqrt{\frac{\mathrm{K}}{\mathrm{E}}}\left[\frac{I_{1}\left(\mathrm{r} \sqrt{\left.\frac{\mathrm{E}}{\mathrm{K}}\right)}\right.}{I_{0}\left(\mathrm{a} \sqrt{\frac{\mathrm{E}}{\mathrm{K}}}\right)}\right] \mathrm{z}
\end{aligned}
$$

It is to be noted that all of the normal stresses are essentially equal when Poisson's ratio is close to one-half. These expressions are plotted in Figures 6 and 7 where the strong dependency upon Poisson's ratio is quite evident. To show this more clearly, and because it is a very useful tool for later discussions of experimental results, 


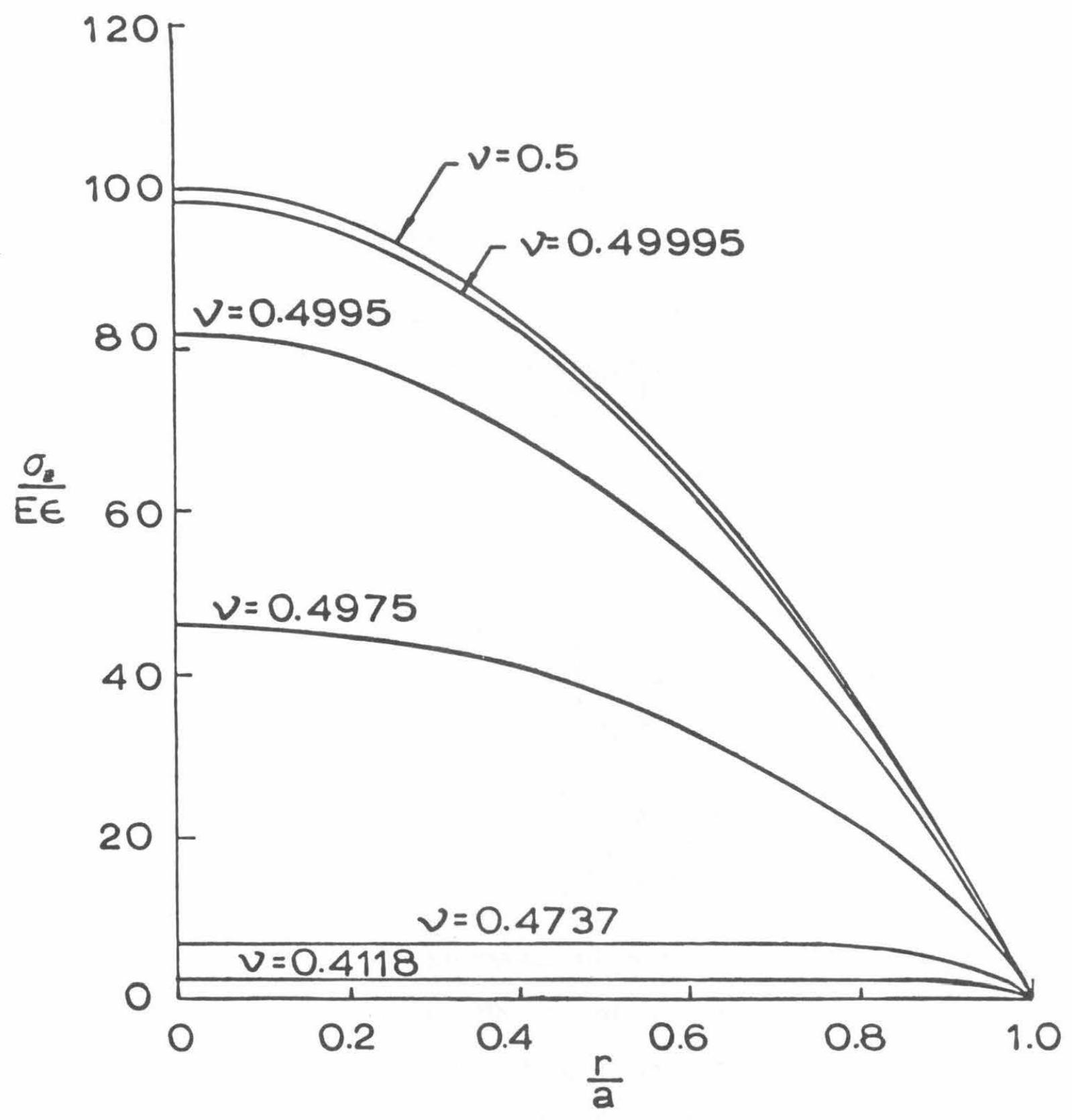

Figure 6. z-Normal Strese in Diok ve, rla: a = 20. 


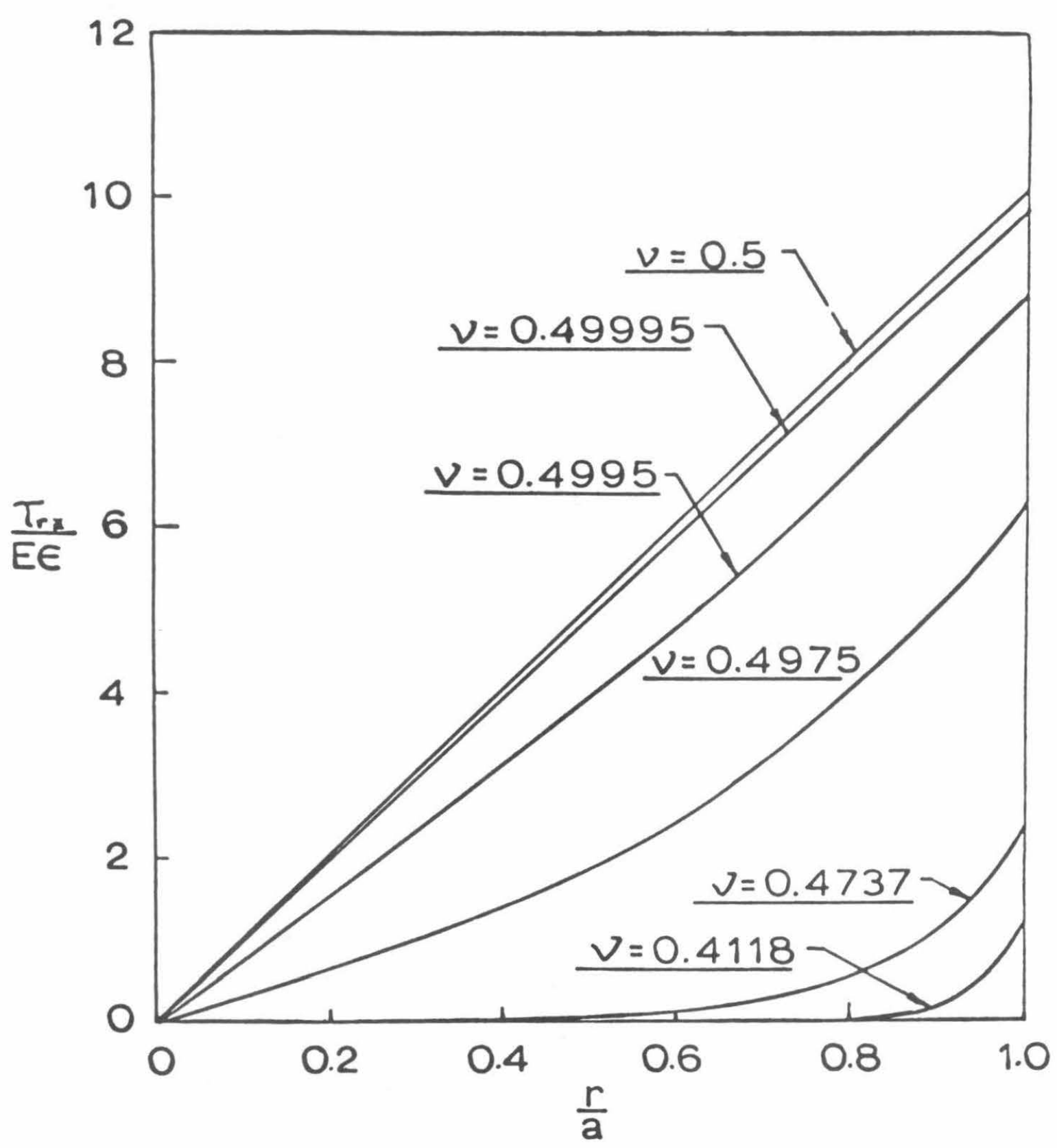

Figure 7. Shear Stree in Diak ve. r/a: $z=1, a=20$. 


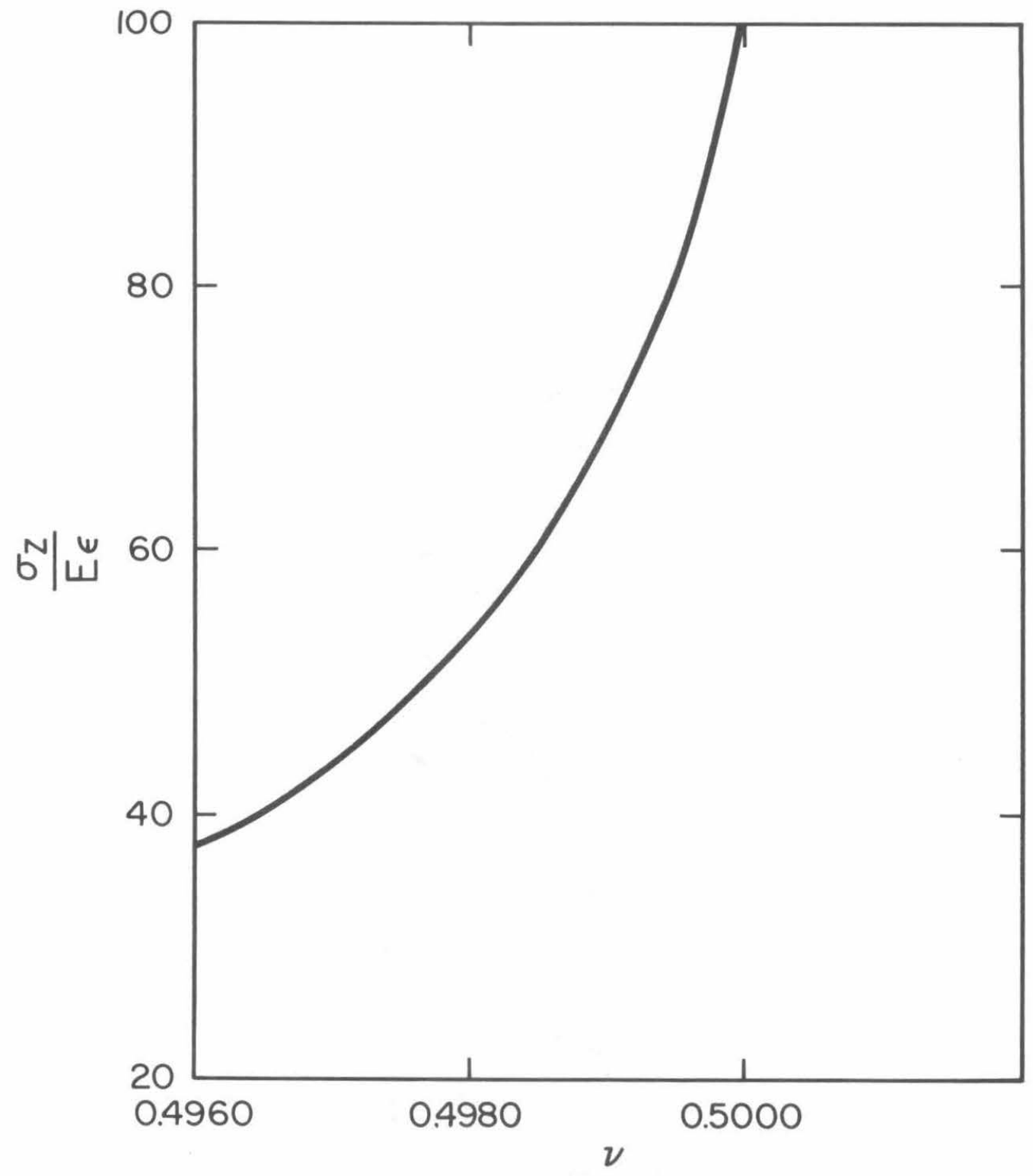

Figure 8. z-Normal Stress in Disk vs. Poisson's Ratio at $\mathrm{r}=0, \mathrm{a}=20$. 
a cross-plot of Figure 6 is given in Figure 8 , where the relationship of local stress to applied strain is shown for $r=0$.

Strictly speaking the only point of true HT is at the midplane $\mathrm{z}=0$; however as seen in Figure 7 the shear stress at the rigid boundary, which is the region of maximum shear, is only a small percentage of the normal stresses. Consequently for all practical purposes the stress field can be considered HT as far out as $r=0.5 a$ with very little error. Furthermore for materials with Poisson's Ratio down to 0.4975 or below, there is an appreciable central region of virtually constant hydrostatic stress, which contributes to the ease and accuracy with which data can be reduced. Large stress gradients make it difficult to know with precision what the local fracture levels actually are.

Displacement Expression

In addition to the stresses (2.17), the radial displacement,

$$
\begin{aligned}
u & =-g\left(1-z^{2}\right)=-\frac{3 v \epsilon K \sqrt{M} I_{1}(r \sqrt{M})\left(1-z^{2}\right)}{E\left[I_{0}(a \sqrt{M})-\frac{2}{3} M \frac{I_{1}(a \sqrt{M})}{a \sqrt{M}}\right]} \\
& \cong-3 v \epsilon \sqrt{\frac{K}{E}} \frac{I_{1}\left(r \sqrt{\frac{E}{K}}\right)}{I_{0}\left(a \sqrt{\frac{E}{K}}\right)}\left(1-z^{2}\right)
\end{aligned}
$$

is of importance since its midplane value at the boundary, $r=a$, can be measured experimentally, and possibly used to deduce the bulk modulus in view of the sensitivity to Poisson's ratio shown in Figure 9. Furthermore, as shown in Figure 9, this displacement 


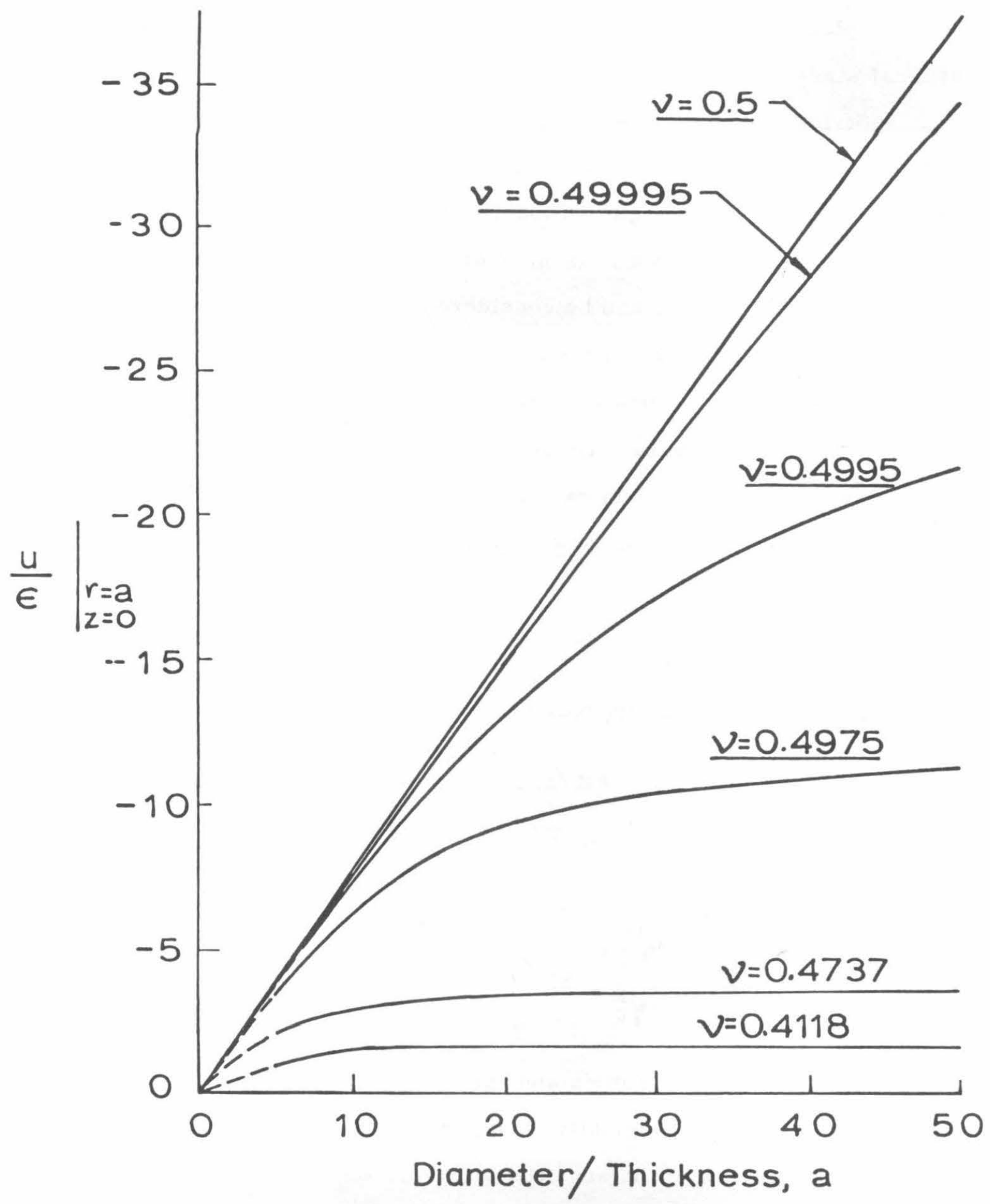

Figure 9. Maximum Radial Displacement in Disk vs. a. 
may be very large for nearly incompressible materials, even when the nominal strain, $\epsilon$, is small. In the neighborhood of the radii for which the radial displacement is not small relative to the disk thicknesses, some error due to large strains will be introduced in the results of the present linear analysis.

\section{Limit-Check of the Solution}

In the incompressible limit case $(v=1 / 2)$ solutions are readily found from equations $(2.16)$ and $(2.18)$ to be

$$
\begin{aligned}
& \frac{\sigma_{r}}{E \epsilon}=\frac{\sigma_{\theta}}{E \epsilon}=\frac{1}{4}\left(a^{2}-r^{2}\right) \\
& \frac{\sigma_{z}}{E \epsilon}=\frac{1}{4}\left(a^{2}-r^{2}\right)+1 \\
& \frac{\tau_{r z}}{E \epsilon}=\frac{r z}{2}
\end{aligned}
$$

where it may be noted that for a large aspect ratio, the condition $(r=0)$ of triaxial hydrostatic tension is achieved. Further,

$$
u=-\frac{3}{4} \epsilon r\left(1-z^{2}\right)
$$

Apparent Modulus

Another quantity of experimental interest is the apparent uniaxial modulus $\mathrm{E}_{\mathrm{A}}$, defined as the ratio of the average stress over the bonded surface $\sigma_{z A}$, required to produce the axial displacement $w$, to the nominal axial strain $\epsilon$, viz.

$$
\mathrm{E}_{\mathrm{A}} \equiv \frac{\sigma_{\mathrm{zA}}}{\epsilon}=\frac{2 \pi \int_{\mathrm{O}}^{\mathrm{a}} \bar{\sigma}_{\mathrm{z}} \mathrm{rdr}}{\pi \mathrm{a}^{2} \epsilon}
$$


Upon substitution of $\sigma_{z}$, equation $(2.16 \mathrm{c})$, into $(2.20)$ we find

$$
\begin{aligned}
\frac{E_{A}}{E} & =\frac{3 v}{1+v}\left(\frac{K}{E}\right)\left[1-\frac{2 I_{1}(a \sqrt{M})}{a \sqrt{M} I_{0}(a \sqrt{M})}\right] \\
& \left.+\frac{1}{1+v}\left[1+\frac{2 I_{1}(a \sqrt{M}) 1-\frac{1}{a \sqrt{M} I_{0}(a \sqrt{M})}}{a \sqrt{M} I_{0}(a \sqrt{M})\left[1+\frac{1-2 v}{v}\left(1-\frac{I_{1}(a \sqrt{M})}{a \sqrt{M} I_{0}(a \sqrt{M})}\right)\right.}\right]\right]
\end{aligned}
$$

This apparent modulus can be conveniently employed for determining the bulk modulus of nearly incompressible materials. Namely, given an aspect ratio $\underline{a}$, and experimentally measured modulus $E_{A}$, the modulus ratio $E / K$ can be deduced from a graph of equation (2.21), such as shown in Figure 10. It is observed that $\mathrm{E}_{\mathrm{A}} / \mathrm{Ea}^{2}$ depends on only the parameter $a \sqrt{\mathrm{E} / \mathrm{K}}$ for $a \simeq 30$. The accuracy of expression $(2.21)$ is expected to be good, even for small aspect ratios. This follows from the fact that the apparent modulus is an average property, and therefore should not be sensitive to error in stress near the periphery. Furthermore, $\mathrm{E}_{\mathrm{A}}$ has the correct limiting value of $\mathrm{E}$ for $\mathrm{a}=0$.

End Effect Parameter

By forming a ratio of expression (2.21) and (2.17b) a measure of the multiplicity of the local hydrostatic stress over the average applied stress can be obtained.

$$
\frac{\sigma_{\mathrm{z}}}{\mathrm{E} \epsilon} / \frac{\mathrm{E}_{\mathrm{A}}}{\mathrm{E}}=\sigma_{\mathrm{z}} / \sigma_{\mathrm{A}}
$$




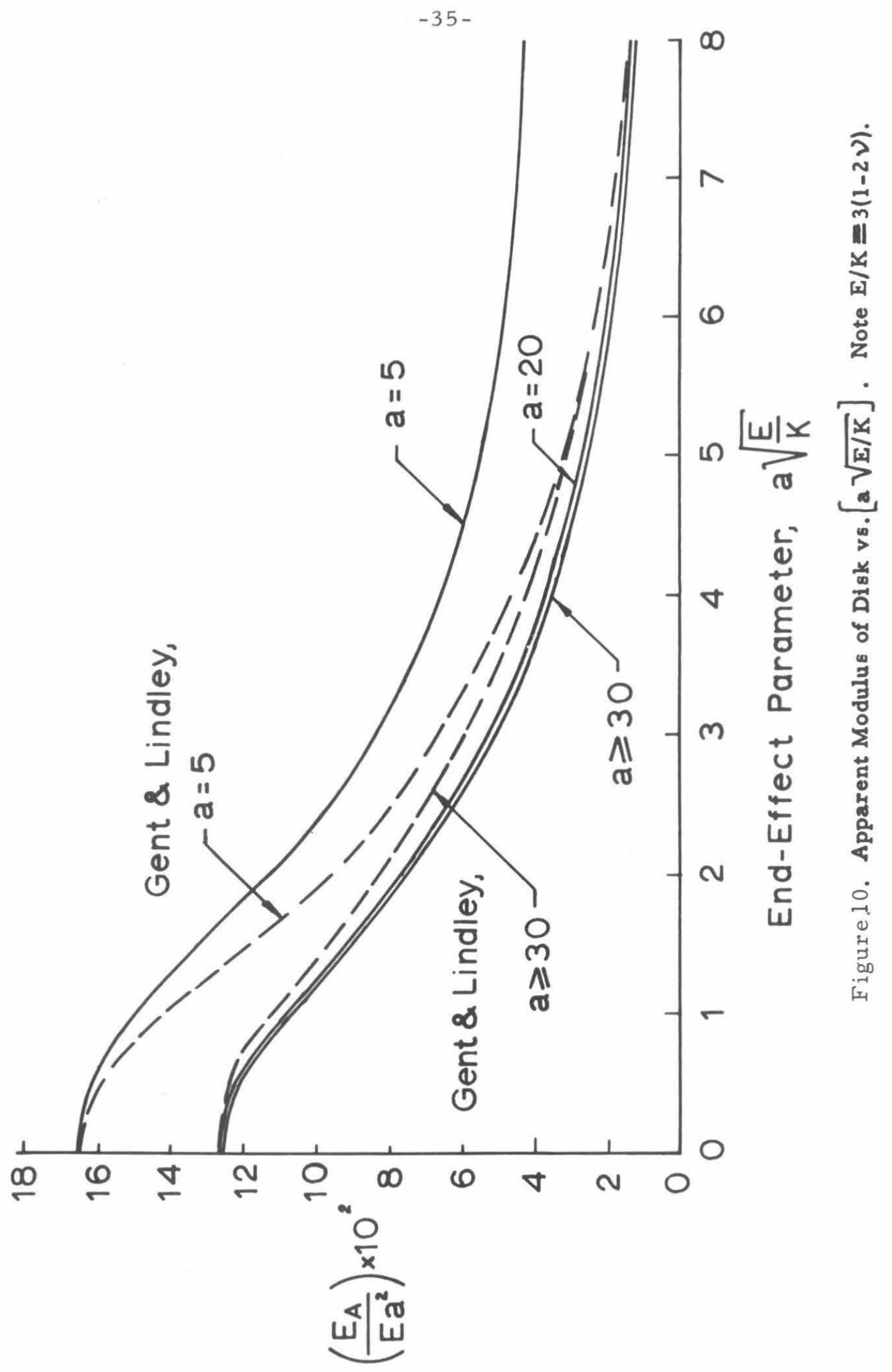


By evaluating this quantity at a given location in the specimen, the effect of aspect ratio can be plotted versus material parameters. Figure 11 is the result for the center of the poker-chip and is quite useful as an aid for acquiring an intuitive feel of the geometrical effects. The abscissa is plotted for values of a greater than or equal to about fifteen. In other words the limit conditions for $\mathrm{a} \sqrt{\mathrm{E} / \mathrm{K}} \rightarrow 0$ would represent an incompressible material, not a uniaxial tensile specimen. For most elastomers the ratio of maximum normal stress to the average applied stress will be in the range of 1.8 to 2.0 .

The Effect of Corner Stress Singularities

The methods of solution just presented for the stresses in the poker-chip are not able to predict the conditions at the boundaries where the character of the boundary conditions change, the reason being the presence of stress singularities, which give rise to large gradients of stress that become averaged out by the global methods used. Therefore this region has to be investigated by a different method capable of describing the local character of the field variables. Such a method was employed by Williams $(42,43)$ in studies of plates with angular corners, and then extended by him to include bimaterial systems. (44) Zak (45) showed that when the methods employed by Williams are extended to bodies of revolution the same results are obtained as in the case of plane strain. Therefore Lindsey and Zak (46) obtained the solution to the poker-chip problem through the use of the plane strain configuration of Figure 12. 


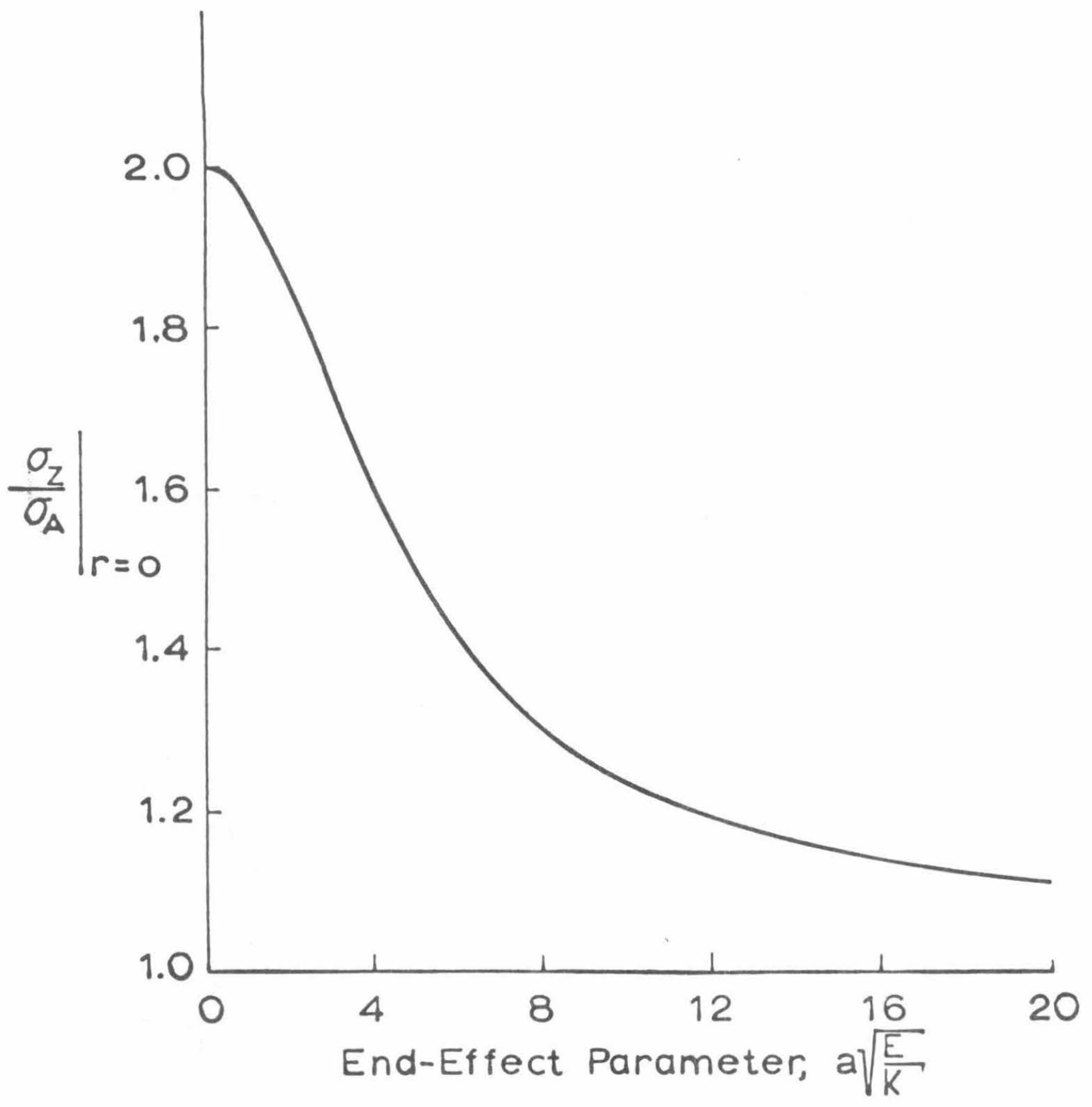

Figure 11. Ratio of Maximum Normal Stres 6 in Disk to Average (Applied) Stres ve. a $\sqrt{\mathrm{E} / \mathrm{K}:} \mathrm{E} / \mathrm{K} \ll<1$. 


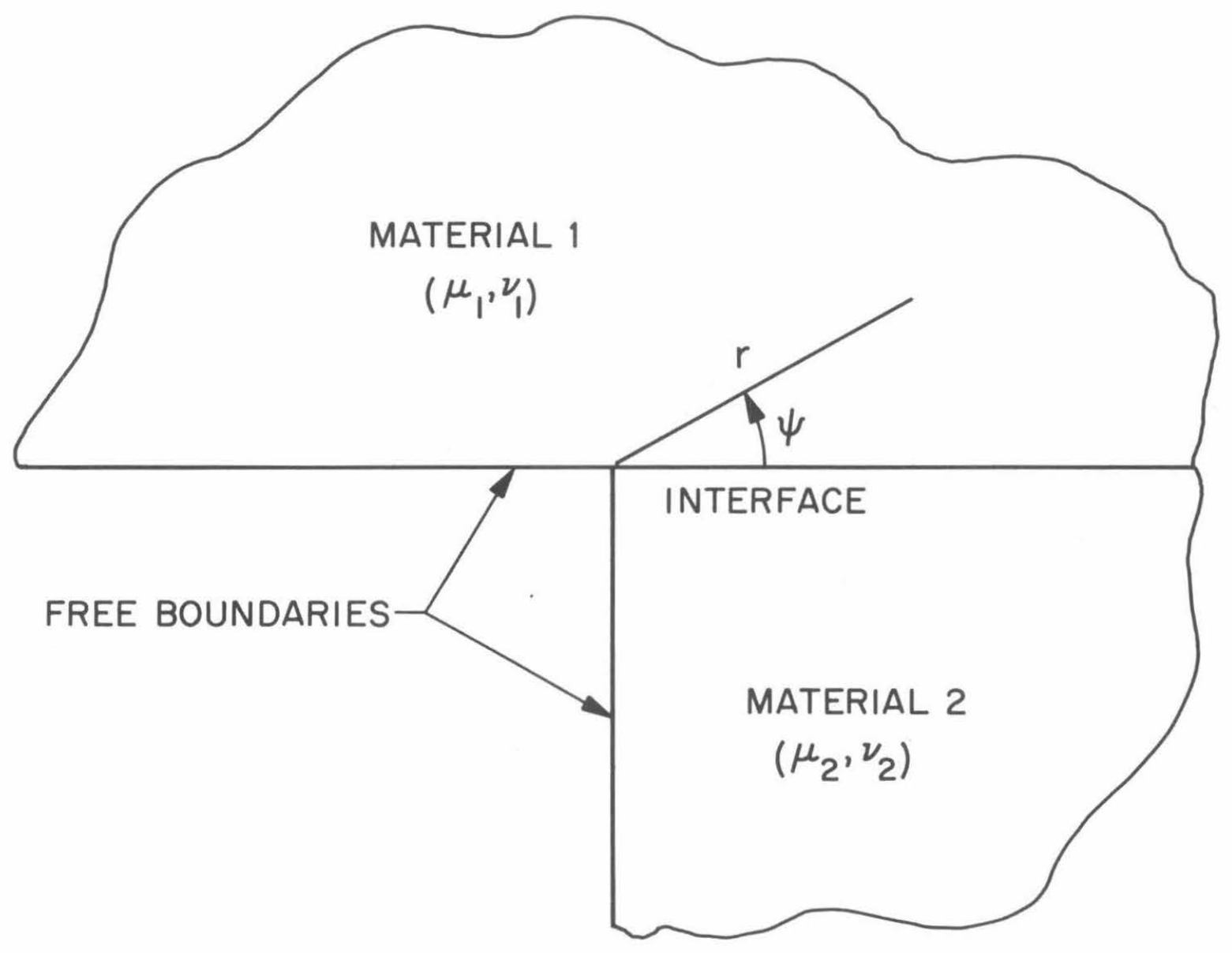

Figure 12. Mathematical Model with Coordinate Description for the Investigation of the Stress Singularity. 
This represents the condition existing at the junction of the free and rigid boundaries, as shown in Figure 5. Material 1 represents the grips to which the poker-chip specimen is bonded and Material 2 represents a portion of the specimen near the edge. Material 1 extends over $180^{\circ}$ and Material 2 over $90^{\circ}$. This situation corresponds to the case where the grips have a larger diameter than the specimen. A complete analysis of the eigenvalues that produce the stress singularities is given in Reference (41), including a matching of the localized singular stresses to the field stresses obtained by the approximate solution.

\section{COMPARISON OF RESULTS WITH OTHER SOLUTIONS}

Finite Difference

It has been interesting to see how closely the stress distributions obtained from the approximate solution of this section have been verified by the numerical results subsequently obtained in Reference (39). Using equations (2.17) the three average normal stresses and the shear stress have been computed for the case $a=10$ and $v=0.4$ and 0.5 , which are configurations analyzed in Reference (39). The results of this calculation for the axial stress $\sigma_{\mathrm{z}}$ are shown in Figure 13, where the stresses obtained from the two methods of solution are compared. It can be seen from these results that, although the analytical method predicts only the aver-

age normal stress $\bar{\sigma}_{\mathrm{z}}$, the two methods agree very closely except at the edge of the poker chip. At the edges both methods are not accurate because of the presence of the singularity. The agreement 

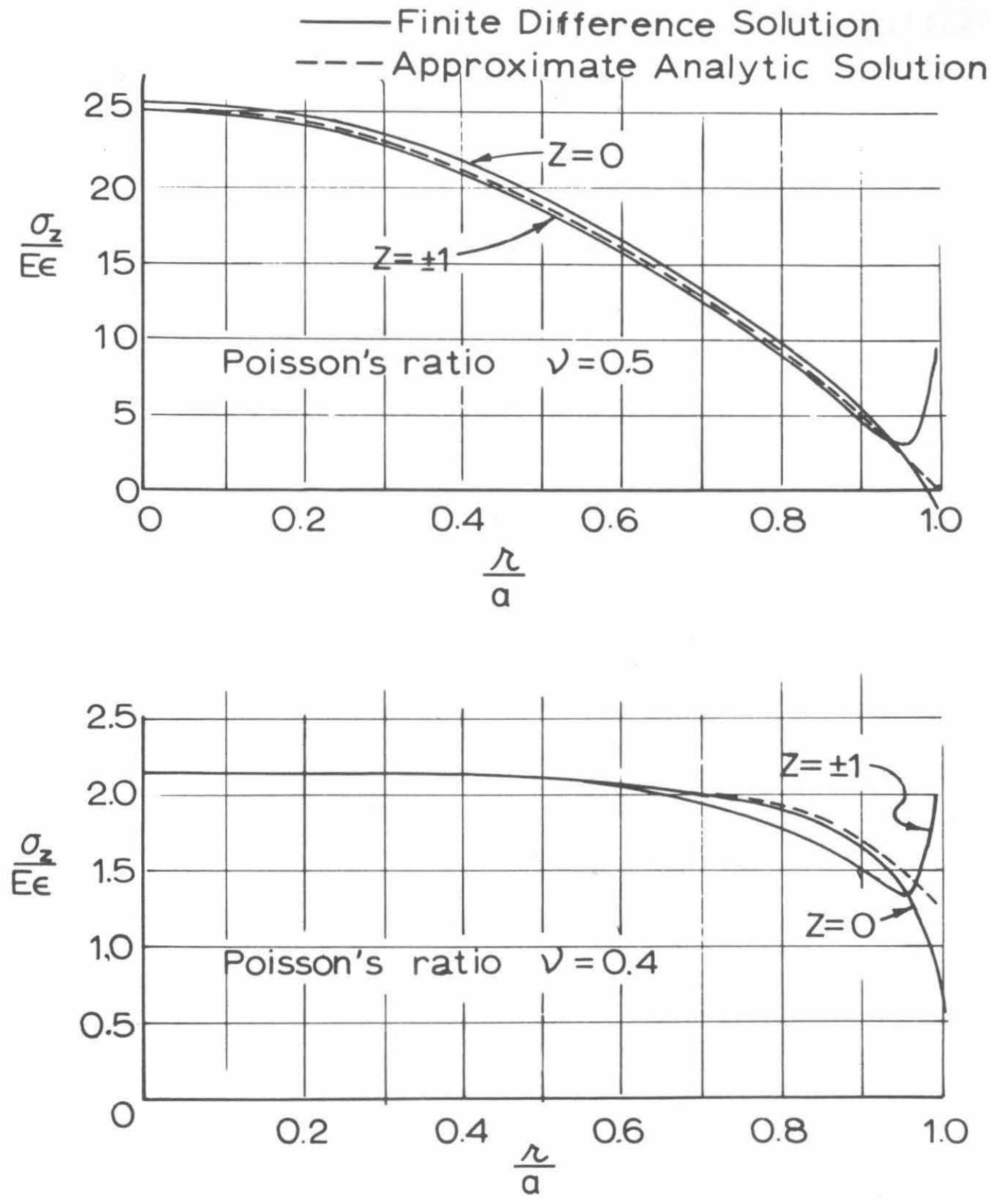

Figure 13. Comparison of Axial Stresses Obtained from Two Different Methods of Solution. 
for the other three stresses is equally good.

Potential Energy Analysis

In a previous section, an analysis was presented which satisfied the displacement boundary conditions, and the z-averaged equilibrium equations and stress boundary conditions. As such the solution was expected to be a judicious compromise between a best deformation (minimum potential energy) and best stress (minimum complementary energy) approximation. It is informative to inquire at this point what type solution would result if the potential energy were minimized, particularly as the deformation functions chosen earlier, i.e. (2.7), are admissible functions for application of this theorem. It will be convenient for later purposes to use the dimensionless forms, viz.

$$
\begin{aligned}
& u(r, z)=-\left[1-\left(z / h_{v}\right)^{2}\right] g(r) \\
& w(z)=\left(w_{0} / h_{v}\right) z
\end{aligned}
$$

where $h_{v}$ is the half-thickness of the specimen.

In the absence of body forces and with zero applied stress on the stress prescribed boundary $\mathbf{r}=\mathrm{a}$, the Minimum Potential Energy Theorem (47) requires that the potential energy

$$
\mathrm{V}=\int_{0}^{\mathrm{a}} \int_{-\mathrm{h} v}^{\mathrm{h} v}\left\{\frac{\lambda}{2}\left[\epsilon_{\mathrm{r}}+\epsilon_{\theta}+\epsilon_{\mathrm{z}}\right]^{2}+\mu\left[\epsilon_{\mathrm{r}}^{2}+\epsilon_{\theta}^{2}+\epsilon_{\mathrm{z}}^{2}+\frac{\mathrm{v}_{\mathrm{rz}}}{2}\right]\right\} \mathrm{dzr} \mathrm{dr} \cdot 2 \pi
$$

be a minimum with respect to the variation of functionals involved in the double integral. Using the expressions for strains (2.8) in 
(2.24) and performing the variation by standard techniques, one finds the governing differential equation to be

$$
g^{\prime \prime}(r)+\frac{1}{r} g^{\prime}(r)-\left[\frac{1}{r^{2}}+M_{v}\right] g(r)=0
$$

where

$$
M_{v} \equiv \frac{5}{4} \cdot \frac{1-2 v}{1-v} \cdot \frac{1}{h_{v}^{2}}
$$

The boundary condition turns out to be

$$
\mathrm{g}^{\prime}(\mathrm{a})+\frac{v}{1-v} \frac{\mathrm{g}(\mathrm{a})}{\mathrm{a}}=\frac{5}{4} \frac{v}{1-v} \frac{\mathrm{w}_{\mathrm{o}}}{\mathrm{h}_{\mathrm{v}}}
$$

The appropriate solution of $(2.25)$ is for finite displacements at the center of the specimen, $r=0$,

$$
g(r)=A_{v_{O}}\left(r \sqrt{M_{v}}\right)
$$

where to satisfy $(2.26)$

$$
A_{v}=\frac{5 v}{4} \frac{w_{0} a}{h_{v}} \frac{1}{(1-v) a \sqrt{M_{v}} I_{0}(a \sqrt{M})-(1-2 v) I_{1}\left(a \sqrt{M_{v}}\right)}
$$

The basic similarity with (2.12), the z-averaged method, is very evident. For $h_{v}=1$, a 5:6 ratio for $M_{v}: M$ is obtained in the governing equation and nearly the same ratio is found for $A_{v}: A_{\text {. In }}$ the latter the ratio is not precisely 5:6 because $A$ is a function of M. Nevertheless a qualitative idea of the difference in the two and indirectly a bound on the average error can be obtained by observing from the differential equation that 


$$
\frac{M_{v}}{M}=\frac{5}{6} \cdot \frac{1}{h_{v}^{2}}
$$

such that if $h=\sqrt{5 / 6}=0.912$ then the governing equations for a minimum energy solution of $\mathrm{h}_{\mathrm{v}}=0.912$, or $\mathrm{a}_{\mathrm{v}}=1.088$ corresponds to $\mathrm{a}$ $\mathrm{z}$-averaged stress solution in a specimen of thickness $\mathrm{h}=1$ and aspect ratio, a. Of course, if values of $\mathrm{M}_{\mathrm{v}}$ and $\mathrm{A}_{\mathrm{V}}$ from (2.25) and (2.27) are used in the basic solution instead of $M$ and $A$ from (2.13) and (2.15), then the results for the minimum potential energy solution can be immediately reproduced.

Because one is generally interested in the stress state at failure, and hence stresses that satisfy equilibrium of stress at least in some sense - here taken as the $\mathrm{z}$ average - it is recommended that the results from the previous section be used for fracture analysis.

\section{POKER -CHIP SPECIMEN SUBJECTED TO COMBINED TRIAXIAL LOADS}

Having obtained a solution for the hydrostatic tensile field, it is of interest to inquire into the possibility of creating a more general three-dimensional test specimen. One such method has been suggested by Lindsey (48) which employs an adaptation of the poker-chip test to a state of combined loading, wherein a shear producing torque, as well as axial tension, is applied to the disk through the rigid plates. Such a test is theoretically feasible and has the capability of producing a general triaxial field, but it has never actually been attempted in the laboratory. The solution is given here for completeness. 
The idea is to superpose upon the axial extension of the pokerchip a torque about its longitudinal axis that will produce a shift in the magnitude and direction of the principal stresses. The result will be a general triaxial stress state with which failure surfaces can be more definitely described and with which the actual failure mechanisms can be studied. With this modification of the pokerchip test, a wide range of stress fields can be obtained by varying the ratio of angle of twist to axial extension. Consequently, a study can be made of the change, or constancy, in the appearance, location, orientation and initiation level of the initial fracture point.

Torsion of a Circular Cylinder (49)

The definition of the stress field results from combining the stress fields of pure extension and that of torsion of a circular cylinder. It will be recalled from classical theory of elasticity that for a circular cylinder (and only a circular cylinder) a solution to the torsion problem can be obtained which leaves the lateral surfaces free of stress and does not warp the cross-section. The amount of rotation of a point in a cross-section depends upon its distance from a base of reference which we will take to be $z=+1$ from Fig. 5 .

$$
\theta=a(z-1)
$$

where $a$ is the twist per unit length. For a pure torque, the only displacement is

$$
\mathrm{v}_{\theta}=\mathrm{r} \theta=\mathrm{ra}(\mathrm{z}-1)
$$


The resulting strains are

$$
\gamma_{\theta z}=r a \quad \varepsilon_{r}=\varepsilon_{\theta}=\varepsilon_{z}=\gamma_{r \theta}=\gamma_{r z}=0
$$

The corresponding system of stresses is

$$
\tau_{\theta z}=\operatorname{Gra} \quad \sigma_{r}=\sigma_{\theta}=\sigma_{z}=\tau_{r \theta}=\tau_{r z}=0
$$

This solution will now be used in conjunction with the approximate analytical solution for the poker chip in extension.

Stress Analysis of Combined Torsion and Extension

Following a procedure very similar to the one used previously on the regular poker chip, the displacement functions are assumed to be,

$$
\begin{aligned}
& u_{r}=-\left(1-z^{2}\right) g(r) \\
& v_{\theta}=r a(z-1) \\
& w=\varepsilon z
\end{aligned}
$$

In (2.33) the displacement boundary conditions are satisfied at surfaces $z= \pm 1$ and $g(r)$ is presently an unprescribed function of the radius. The strains corresponding to these displacements are found to be

$$
\begin{aligned}
& \varepsilon_{r}=\frac{\partial u_{r}}{\partial r}=-\left(1-z^{2}\right) g^{\prime}(r) \\
& \varepsilon_{\theta}=\frac{u_{r}}{r}=-\left(1-z^{2}\right) \frac{g(r)}{r} \\
& \varepsilon_{z}=\frac{\partial w}{\partial z}=\varepsilon
\end{aligned}
$$




$$
\begin{aligned}
& \gamma_{r \theta}=\frac{\partial v_{\theta}}{\partial r}-\frac{v_{\theta}}{r}=0 \\
& \gamma_{\theta z}=\frac{\partial v_{\theta}}{\partial z}=r a \\
& \gamma_{r z}=\frac{\partial u_{r}}{\partial z}=2 z g(r)
\end{aligned}
$$

from which the $\mathrm{z}$ averaged normal stresses are found as

$$
\begin{aligned}
& \bar{\sigma}_{r}=\frac{1}{2} \int_{-1}^{1} \sigma_{r} \mathrm{dz}=\lambda\left(\varepsilon-\frac{2}{3} g^{\prime}(r)-\frac{2}{3} \frac{g(r)}{r}\right)-\frac{4}{3} G^{\prime}(r) \\
& \bar{\sigma}_{\theta}=\frac{1}{2} \int_{-1}^{1} \sigma_{\theta} d z=\lambda\left(\varepsilon-\frac{2}{3} g^{\prime}(r)-\frac{2}{3} \frac{g(r)}{r}\right)-\frac{4}{3} G \frac{g(r)}{r} \\
& \bar{\sigma}_{z}=\frac{1}{2} \int_{-1}^{1} \sigma_{2} d z=\lambda\left(\varepsilon-\frac{2}{3} g^{\prime}(r)-\frac{2}{3} \frac{g(r)}{r}\right)+2 G \varepsilon
\end{aligned}
$$

As will be seen from the equilibrium equation, it is not necessary to average the shear stresses from the equilibrium equation,

$$
\begin{aligned}
& \tau_{\text {r } \theta}=0 \\
& \tau_{\mathbf{r z}}=2 \mathrm{Gg}(\mathbf{r}) \mathrm{z} \\
& \tau_{\theta_{\mathbf{z}}}=\mathrm{Gra}
\end{aligned}
$$

The radial equilibrium equation is satisfied on the average as before with the tangential expression becoming,

$$
\frac{1}{2} \int_{-1}^{1} \frac{\partial \tau_{\theta z}}{\partial z} \mathrm{dz}=\frac{1}{2}\left[\tau_{\theta_{z}}\right]_{-1}^{1}=0
$$

However, $\tau_{\theta z}=$ Gra and is not dependent upon z. The third equilibrium equation is satisfied identically due to the symmetry of the 
specimen and applied load about the midplane, $z=0$. Thus the differential equation for $g(r)$ remains unchanged

$$
g^{\prime \prime}(r)+\frac{g^{\prime}(r)}{r}-\left(\frac{1}{r^{2}}+M\right) g(r)=0
$$

where

$$
M=\frac{3 G}{\lambda+2 G}=\frac{3}{2} \frac{(1-2 v)}{(1-v)}
$$

Now it can be seen that the $v_{\theta}$ displacement arising from the torsion portion of the load produces no effect upon any of the field quantities from the approximate solution for the regular poker-chip. Therefore the two loads are superposable just as they would be for exact solution of infinitesimal theory. The normal stresses for the combined loading, valid everywhere except near the edges, become the same as before for a regular poker-chip, equation (2.17), and the shear stresses become,

$$
\begin{aligned}
& \frac{\tau_{\mathrm{rz}}}{\mathrm{E} \varepsilon} \cong \frac{3 v}{1+v} \sqrt{\frac{\mathrm{K}}{\mathrm{E}}}\left[\frac{\mathrm{I}_{1}\left(\mathrm{r} \sqrt{\frac{\mathrm{E}}{\mathrm{K}}}\right)}{\mathrm{I}_{0}\left(\mathrm{a} \sqrt{\frac{\mathrm{E}}{\mathrm{K}}}\right)}\right] \mathrm{z} \\
& \frac{{ }^{\theta_{\mathrm{z}}}}{\mathrm{E}_{\varepsilon}}=\frac{\mathrm{r} \beta}{2(1+v)} \quad \beta=\frac{a}{\varepsilon}
\end{aligned}
$$

\section{Principal Stresses}

By seeking for an orientation of stress such that the surface traction is perpendicular to the surface and no shearing stress exists, one obtains an equation of the form,

$$
\left|\tau_{i j}-\sigma \delta_{i j}\right|=0
$$


where $\sigma$ represents the principal stresses. Expanding the determinant,

$$
\begin{aligned}
& \sigma^{3}-\left(\bar{\sigma}_{\mathbf{r}}+\bar{\sigma}_{\theta}+\bar{\sigma}_{\mathbf{z}}\right) \sigma^{2}+\left(\bar{\sigma}_{\mathbf{r}} \bar{\sigma}_{\theta}+\bar{\sigma}_{\mathbf{r}} \bar{\sigma}_{\mathbf{z}}+\bar{\sigma}_{\theta} \bar{\sigma}_{\mathbf{z}}-\tau_{r \theta^{-\tau}}^{2}{ }_{\theta \mathrm{z}}^{2}{ }^{-\tau_{\mathrm{z}}}{ }^{2}\right) \sigma
\end{aligned}
$$

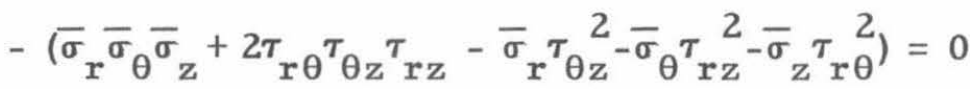

Simplifying to the situation at hand and nondimensionalizing the principal stresses $S=\frac{\sigma}{E_{\varepsilon}}$

$$
\begin{aligned}
S^{3} & -\left(2 \bar{\sigma}_{\mathbf{r}}+\bar{\sigma}_{\mathrm{z}}\right) \frac{\mathrm{S}^{2}}{\mathrm{E} \varepsilon}+\left[\bar{\sigma}_{\mathbf{r}}^{2}+2 \bar{\sigma}_{\mathbf{r}} \bar{\sigma}_{\mathbf{z}}-\left(\tau_{\theta \mathrm{z}}^{2}+\tau_{\mathrm{rz}}^{2}\right)\right] \frac{\mathrm{S}}{(\mathrm{E \varepsilon})^{2}} \\
& -\left[\bar{\sigma}_{\mathbf{r}}^{2} \bar{\sigma}_{\mathrm{z}}-\bar{\sigma}_{\mathbf{r}}\left(\tau_{\theta \mathrm{z}}^{2}+\tau_{\mathbf{r z}}^{2}\right)\right] \frac{1}{\left(\mathrm{E}_{\varepsilon}\right)^{3}}=0
\end{aligned}
$$

Substituting equations (2.17) and (2.38) into this expression and solving the cubic equation for $S$, we obtain the plots of Figs. 14 to 18 .

Observations

There are several things to be noted from this solution, one of which is the fact that for angles of twist that can be classified as being in the range of infinitesimal displacements, the hydrostatic condition can be altered considerably. For example a typical material with $E=500$ psi subjected to $\varepsilon=.005$ and $a=0.1 \mathrm{rad}, \mathrm{S}_{1}=10$ psi, $\mathrm{S}_{2}=92 \mathrm{psi}, \mathrm{S}_{3}=177 \mathrm{psi}$ at $\mathrm{r}=10$. Thus a large variety of stress fields can be readily obtained; however, for failure studies Fig. 16 shows that $\beta>2$ must be used in order to obtain stresses larger than the hydrostatic field in the center. In other words, if 


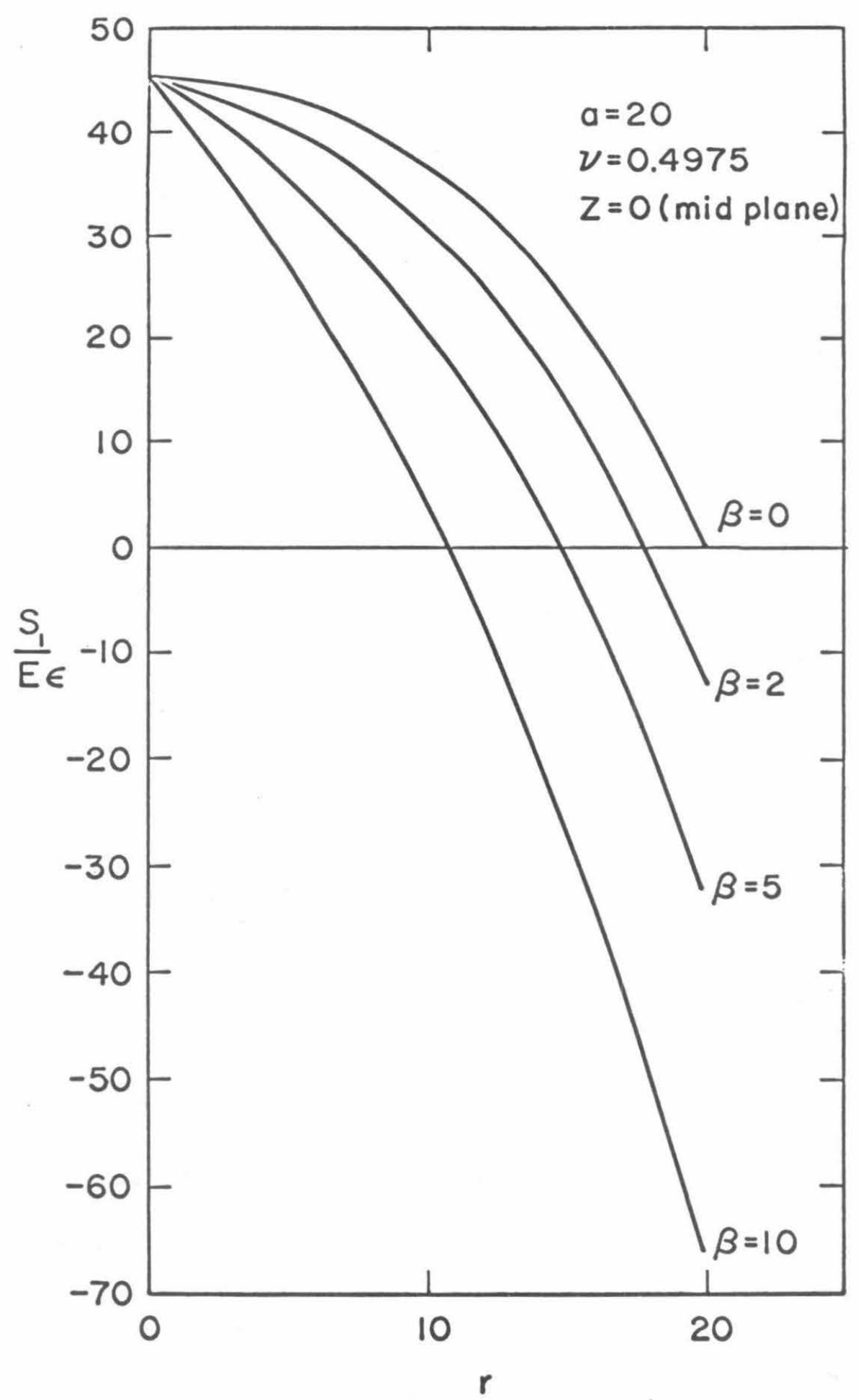

Figure 14. First Principal Stress at Midplane for Modified Poker Chip. ( $\beta=$ angle of twist per unit length/axial strain). 


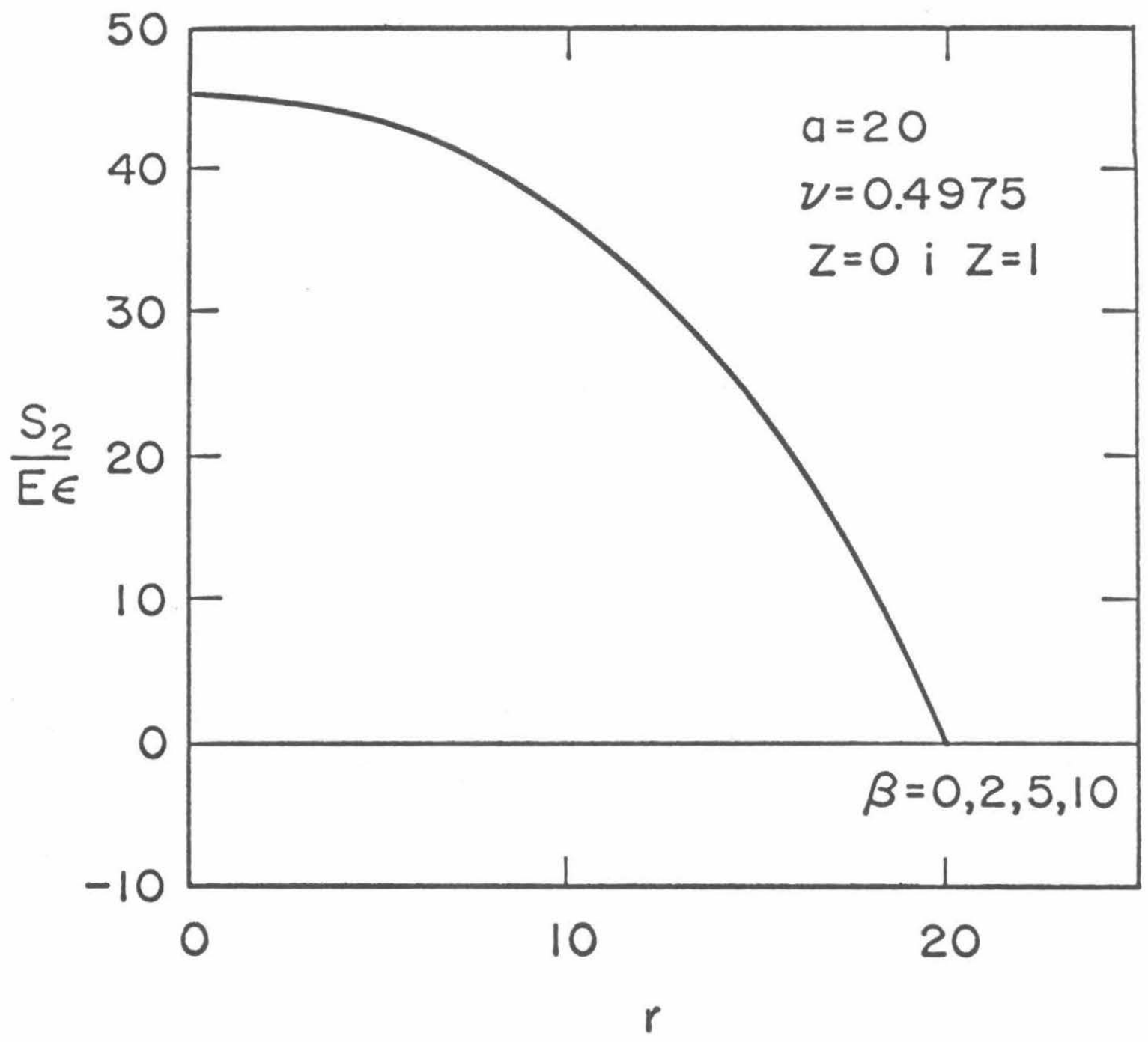

Figure 15. Second Principal Stress at Midplane and Interface for Modified Poker Chip. 
$-51-$

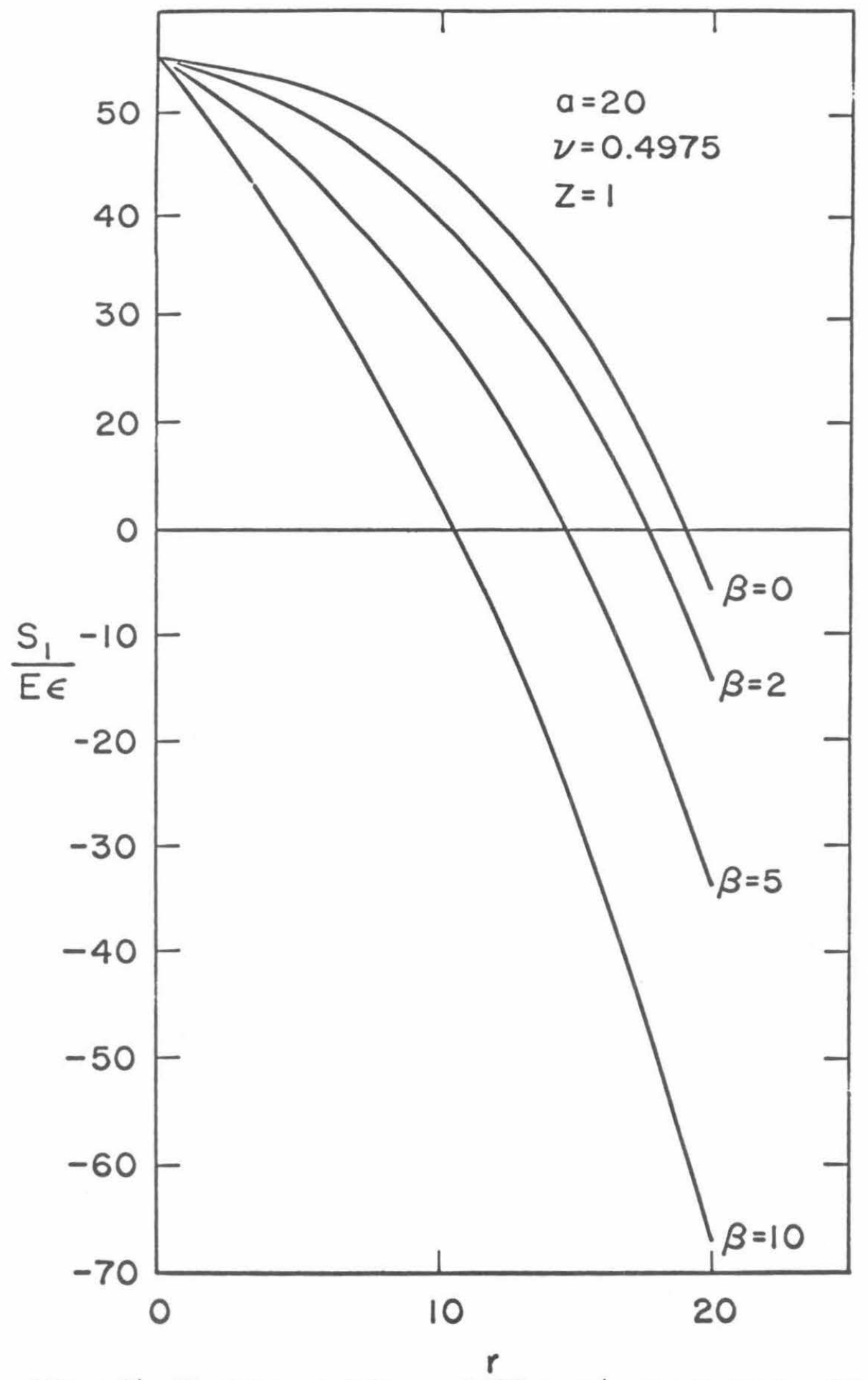

Figure 16. Third Principal Stress at Midplane for Modified Poker Chip. ( $\beta \times$ angle of twist per unit length/axial strain). 


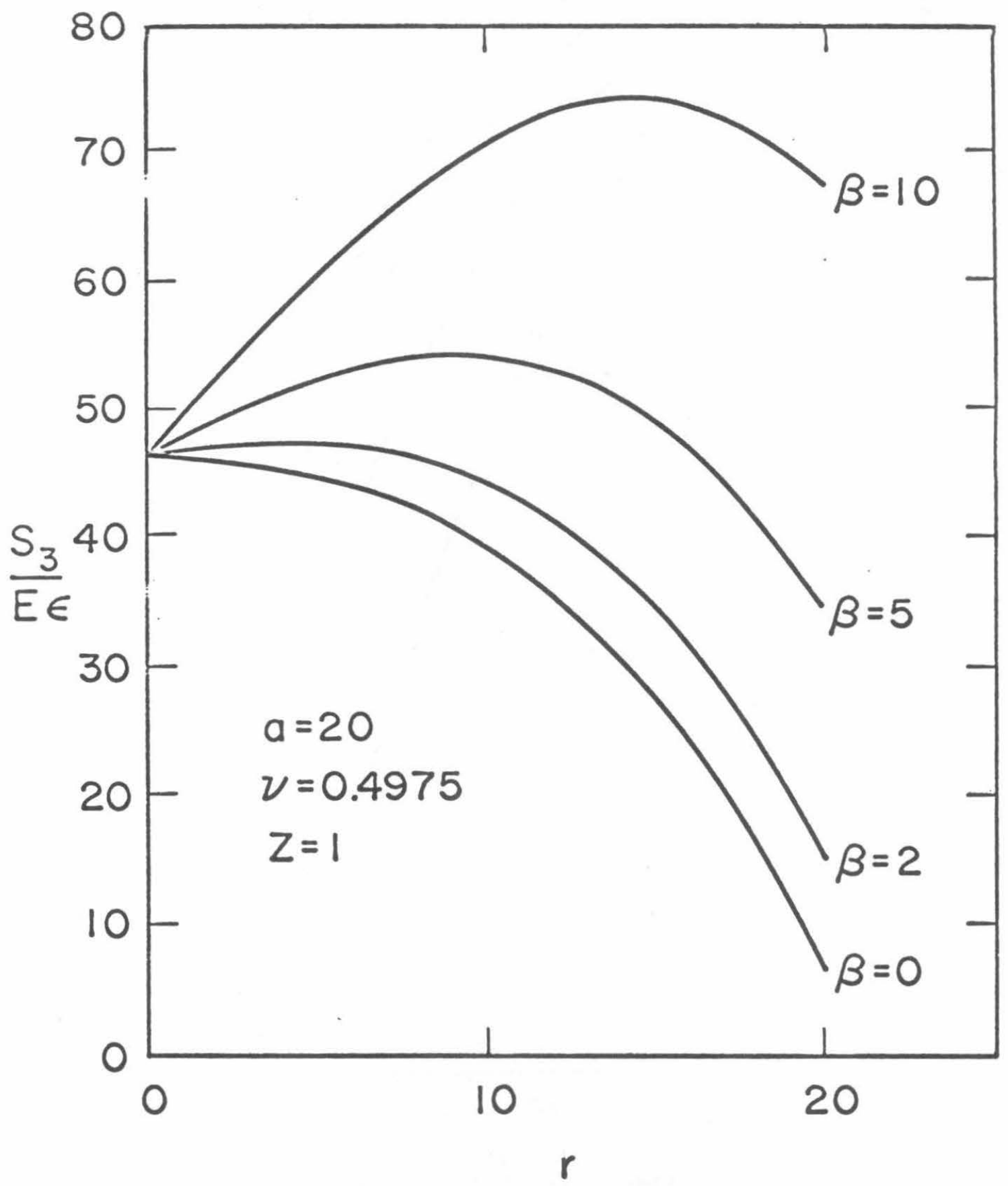

Figure 17. First Principal Stress at Interface for Modified Poker Chip. ( $\beta \times$ angle of twist per unit length/axial strain). 


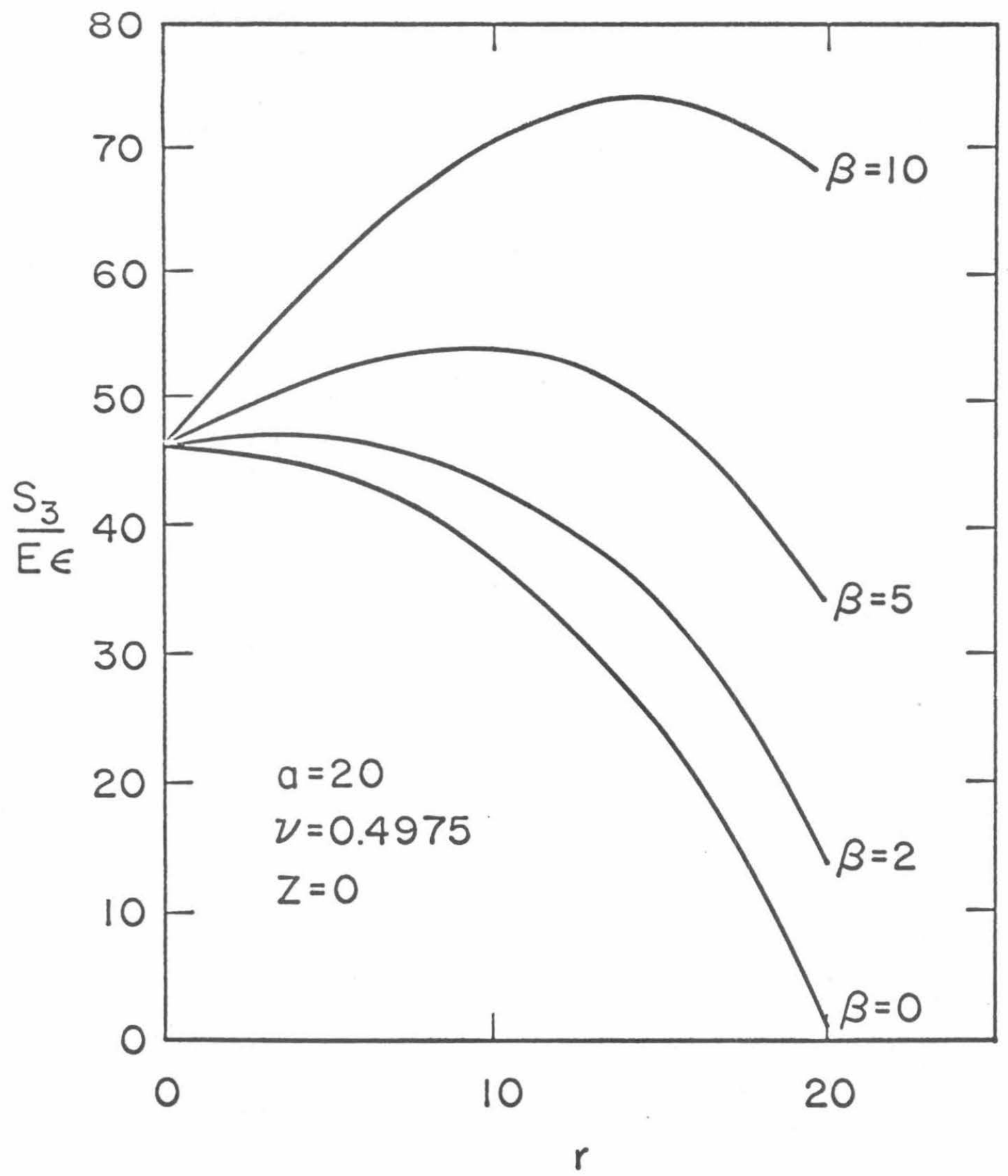

Figure 18. Third Principal Stress at Interface for Modified Poker Chip. ( $\$$ angle of twist per unit length/axial strain). 
$\beta<2$ the largest stresses occur at the center of the specimen where there is always hydrostatic tension, so in order to produce fractures under other conditions $\beta$ must be greater than 2 .

As can be seen from Fig. 17, one component of the triaxial field can be made compressive. This will provide for failure studies in the ++ - quadrant of the failure surface. Little, if any, work has been done in this quadrant because of experimental difficulties, but now barring unforeseen laboratory difficulties this failure surface can be constructed.

One last observation is made from comparing Figs. 15 and 17 and Figs. 16 and 18 . For $\beta>2$ the stress distribution at the midplane is identical to that at the interface of the specimen and the lucite grips. The stress field is virtually constant through the thickness in the central regions a thickness distance in from the edge.

\section{SUMMARY}

In summary it may be stated that these analyses have served to demonstrate the feasibility of producing a state of hydrostatic tension in soft nearly incompressible materials. They have also opened the possibility of creating a rather general state of threedimensional tensile stress, useful in the study of failure surfaces. Furthermore a detailed definition of the field variables has been obtained, suitable for use in the reduction and evaluation of experimental data. As a side benefit, a means for measuring bulk properties in tension has been developed. This is not only a convenient method of obtaining such information, but correlation can 
now be made with compressive values, obtained from the more classical tests, for better definition of material behavior. 
CHAPTER III

EXPERIMENTAL ANALYSIS IN HYDROSTATIC TENSION

MATERIAL DESCRIPTION

The selection, procurement and characterization of an appropriate material areprerequisite to experimental investigation. This always involves a compromise as one tries to find typical materials that are readily available and yet still possessing material properties that are amenable to standard laboratory testing. There is currently in progress a program (50) to find one or more rubber materials suitable as standards for all interested investigators to use as a basis for interchange of information. One of the candidates under study is a polyurethane elastomer of a type employed as propellant binders. It is commercially produced by the Thiokol Chemical Corporation under the trade name of Solithane 113 (S-113). Chemically, urethane polymers are the product of a reaction between an isocyanate and a hydroxyl radical. Normally the process consists of three steps: prepolymer formation, chain extension, and curing. Although the specific formulation of S-113 is company proprietary, some general statements can be made about it. Quoting extensively from 'Polyurethanes: Chemistry and Technology,"' (51) with occasional annotations, we will discuss the three steps.

Prepolymer Formation

The reaction of a disocyanate with a hydroxylterminated polyester, polyester amide, or polyether to form an isocyanate-terminated prepolymer can be represented schematically as follows: 
$\begin{aligned} & \mathrm{OCN}-\mathrm{R}-\mathrm{NCO} \\ & \text { diisocyanate }\end{aligned}+\underset{\text { polyester or polyether }}{\mathrm{HOm}}$<smiles>[R16]NC(=O)O[R]</smiles>

For S-113 the diisocyanate radical $\mathrm{R}$ was Tolilene, TDI. After the basic links are formed, they are used as building blocks to form extended chains.

Chain Extension

Chain extension of the prepolymer with active hydrogencontaining compounds, usually difunctional, such as water, glycols, diamines, or aminoalcohols, proceeds to give a higher molecular weight, soluble polymer. Chain extension with glycols takes place with the formation of urethane groups as shown below:

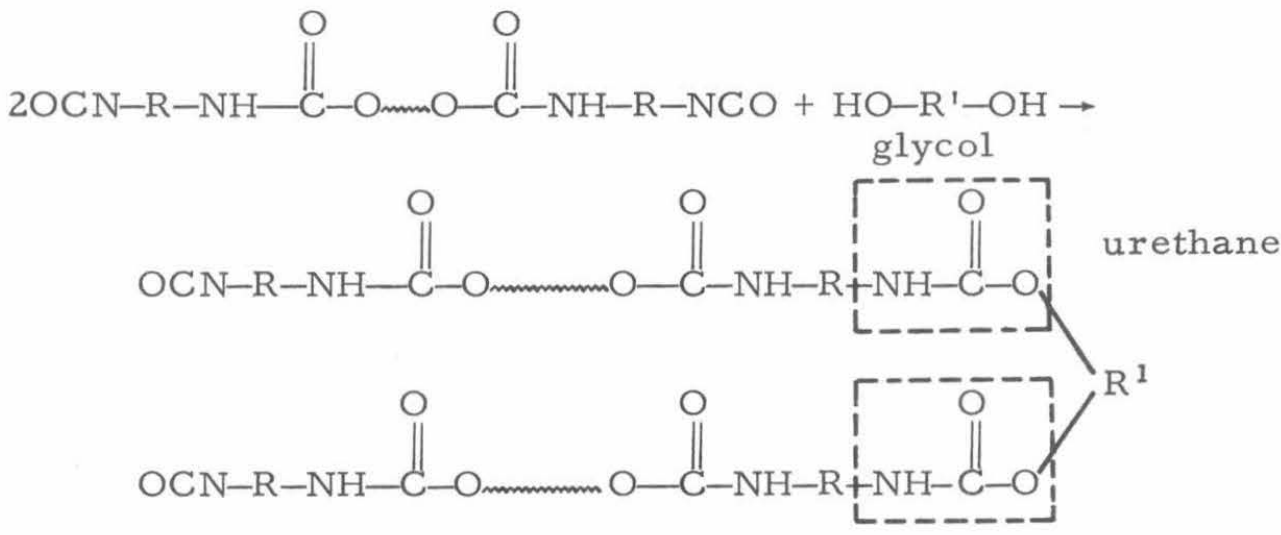

For S-113 the extension agent $\mathrm{R}^{\prime}$ is Polypropylene glycol, PPG.

Thus far chain extension has been shown wherein an excess of isocyanate was used, giving an NCO-terminated polymer. These polymers are actually high molecular weight polyisocyanates, and as such are reactive with many chemicals, hence are not indefinitely stable. Soluble polymers of better stability may be prepared, if desired, by using a slight excess of the active hydrogen 
component, rather than an excess of isocyanate. For example, an excess of glycol would lead to a polyurethane terminating in hydroxyl groups, and would hence be much more stable.

$2 \mathrm{OCN}-\mathrm{R}-\mathrm{NHCOO} m \mathrm{mOCONH}-\mathrm{R}-\mathrm{NCO}+3 \mathrm{HO}-\mathrm{R}^{1}-\mathrm{OH} \rightarrow$<smiles>[R]NC(=O)[R]NC(=O)NNC(=O)OCC([R])(C)O</smiles>

Other active hydrogen compounds could be used similarly, but hydroxyl compounds usually serve as the best chain terminators, free from other complicating side reactions.

Curing or Crosslinking

The curing or crosslinking of the elastomer may be accomplished by reacting an added curing agent with the intermediate molecular weight elastomer, or by formulating the elastomer so that it contains free isocyanate groups and curing by heating.

A convenient means of introducing crosslinking in the urethane polymer chain is the use of triols, either in form of monomeric polyols such as trimetholylpropane or by employing poly(oxypropylene) glycol derivatives of triols such as trimethylolpropane, glycerol, and others. In this case, crosslinking occurs through the formation of urethane links as shown below:

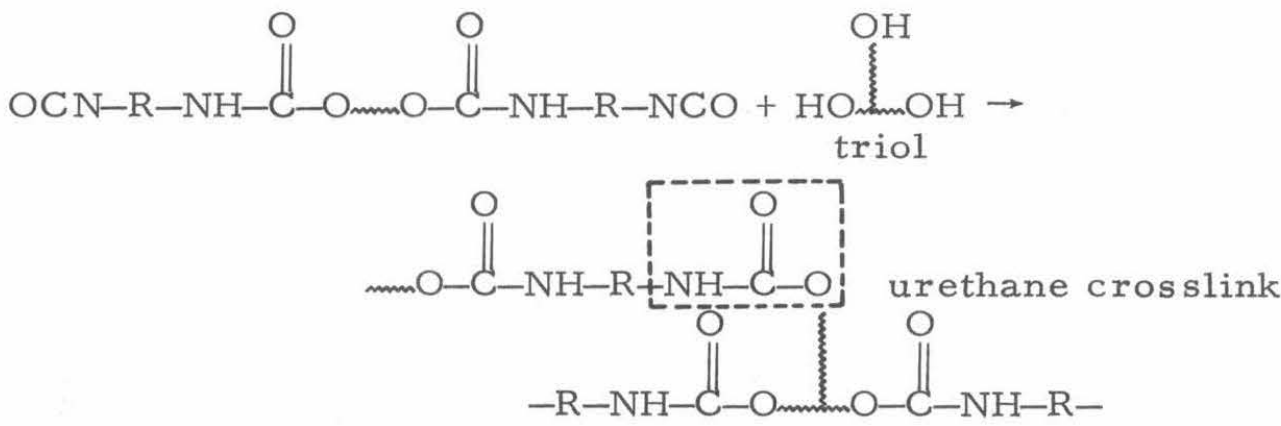

The catalytic triol used in S-113 was Thiokol catalyst C113-300 and curing was prescribed at $150^{\circ} \mathrm{C}$.

S-113 can be made with widely different mechanical properties by varying the relative amounts of the prepolymer and catalyst. 
A study by Knauss (50) shows the degree of sensitivity, which is graphically displayed in Figure 19. The ratio used in this program was one to one by volume, which was found by testing other proportions to be the most suitable for this investigation.

\section{MATERIAL FABRICATION}

A very detailed account of material fabrication along with descriptive photographs and schematics of the equipment has been given by Zak. (51) Briefly the process may be described by stating that the two separate components, prepolymer and catalyst, were preheated to $60^{\circ} \mathrm{C}$ while they were in an inert atmosphere of nitrogen. They were then carefully measured by volume and mixed together, still under nitrogen, and raised to $100^{\circ} \mathrm{C}$. The mix was degassed for five minutes, which acted to reduce virtually to zero the number of visible bubbles produced in the casting, and furthermore it tended to make the finished product more nearly colorless. A mold of polished aluminum was preheated in the oven to $125^{\circ} \mathrm{C}$, and it should be emphasized that no mold release was ever used in the fabrication process. It was found impossible to remove completely the residue left by the release regardless of the solvent used, and the contamination of the surface prevented the formation of a good bond. A polished mold face was found very acceptable for releasing the specimen. Polished brass, steel and aluminum as well as Pyrex glass, and Micarta were used, but polished aluminum was found to be the most desirable when all of the factors of cost, weight, etc. were considered. The surface quality of the 


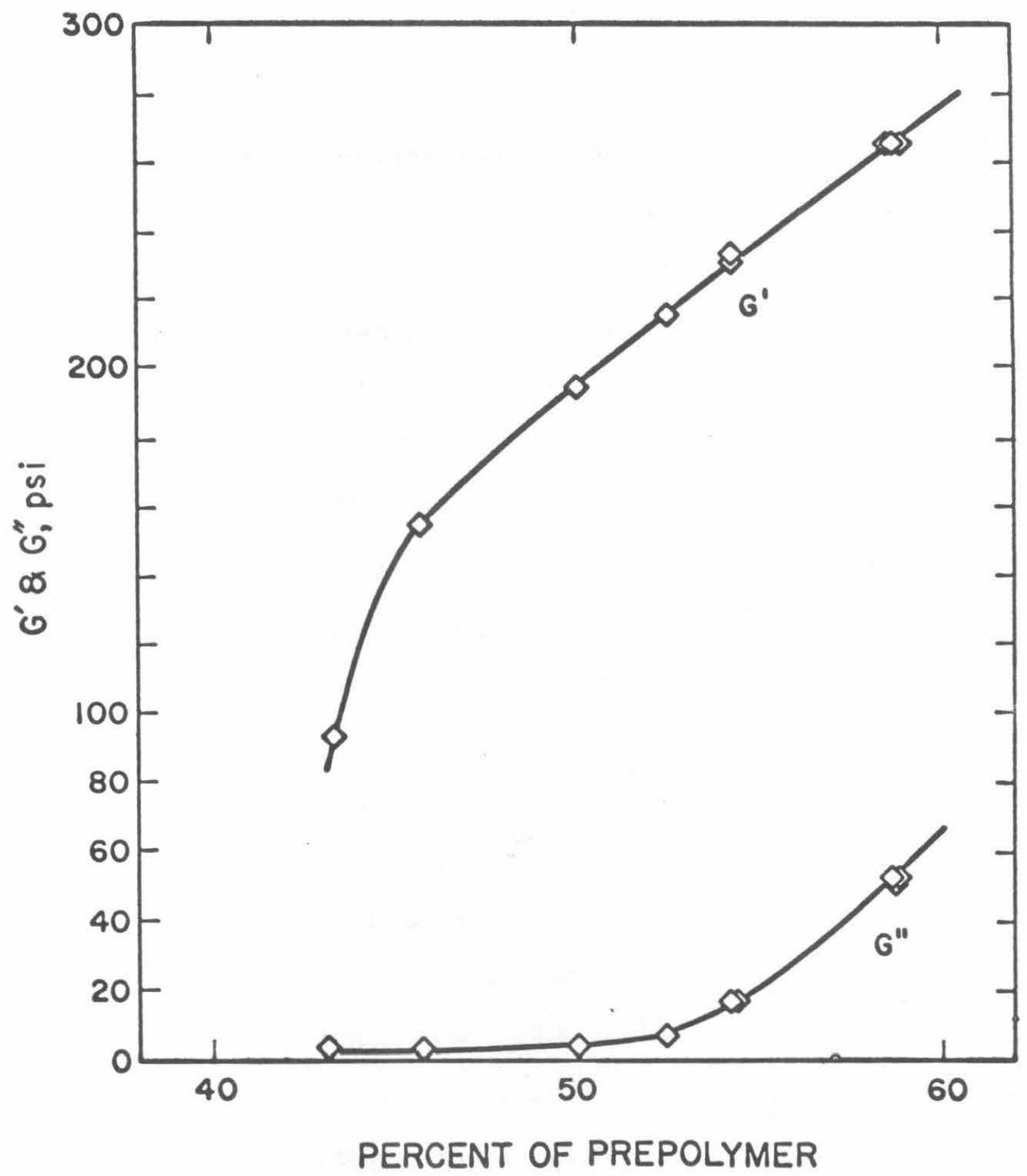

Figure 19a. Complex Modulus at $20^{\circ} \mathrm{C}$ as a Function of Prepolymer-Catalyat Composition. 


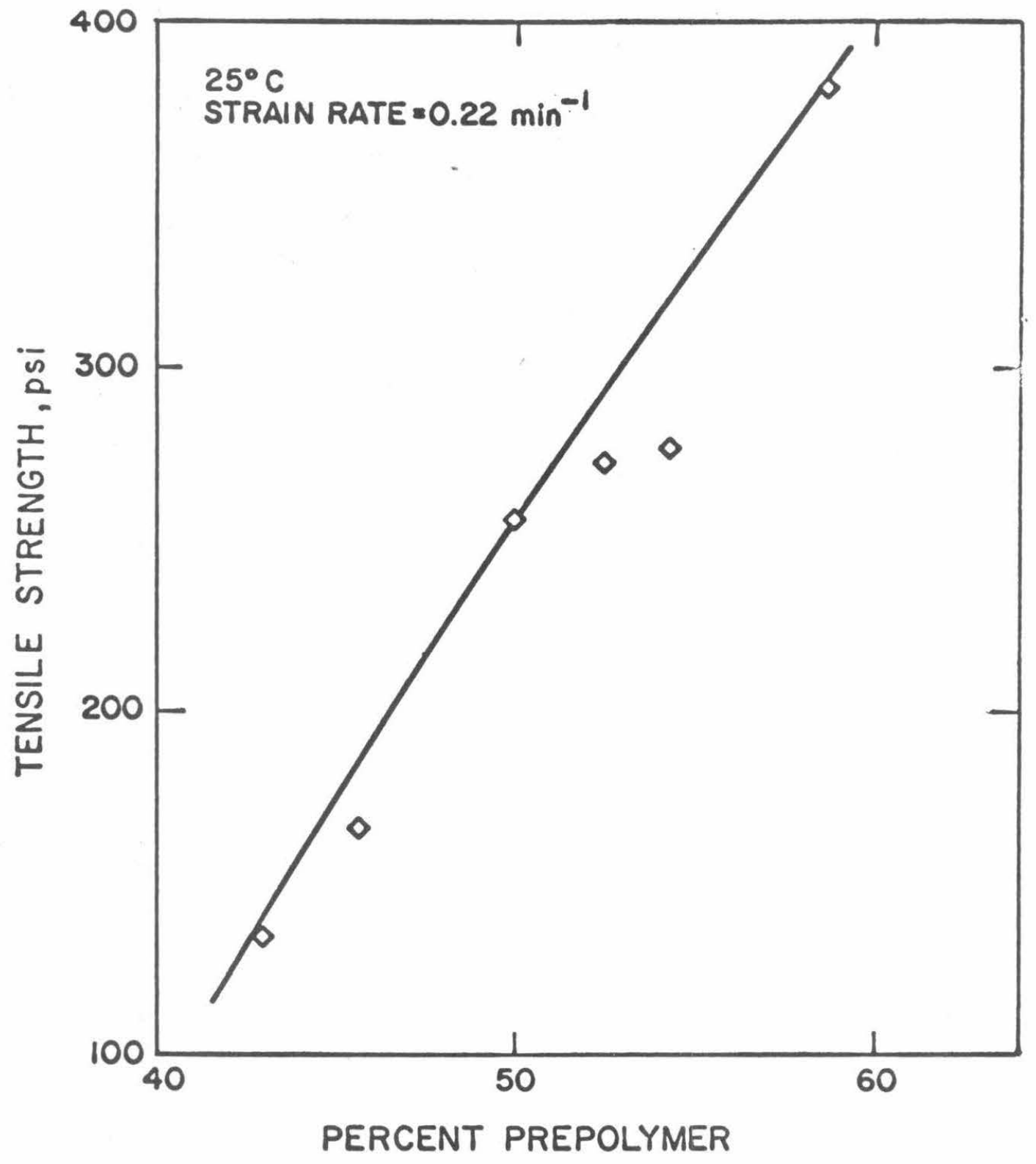

Figure 19b. Uniaxdal Tensile Strength as a Function of Prepolymex-Catalyat Composition at $20^{\circ} \mathrm{C}$. 
cast sheet is directly related to the surface quality of the mold, but as will be discussed later when the material is bonded to the lucite grips, the wetting properties of the bonding agent eradicate surface imperfections in the cast sheet. Therefore the mold surface need be polished only to the degree necessary to allow the rubber to be removed from the mold.

With the mix up to temperature and degassed, the molds were filled through tubular arrangements while they were in the oven, so that the material was never exposed to the atmosphere. The reason for the great care was to prevent side reactions that can be produced by water.

Chain extension with water leads to the formation of substituted urea linkages and the evolution of carbon dioxide as follows: (2)
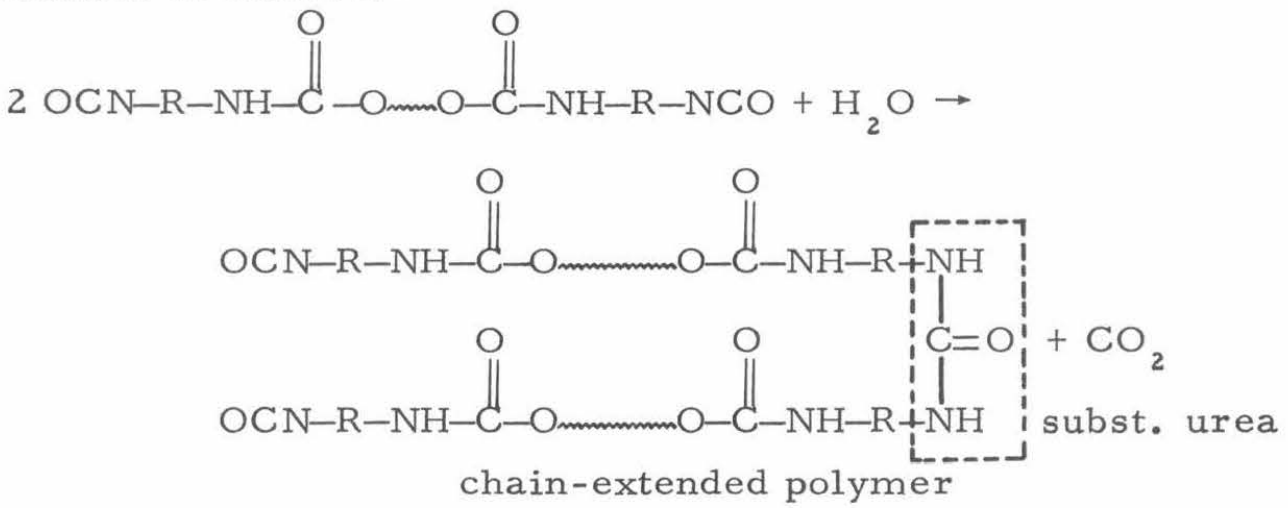

Since such a reaction would cause undesirable by-products like variations in the basic structure and entrapped gases, it is avoided if at all possible. However only small traces of $\mathrm{H}_{2} \mathrm{O}$ can produce the reaction, making it very difficult to control. After filling, the ovens were then raised to $150^{\circ} \mathrm{C}$, and the material was cured for two hours. 
Every effort was made to closely control each step of the process; however due to the fact that several very complex chemical reactions are taking place simultaneously, it is difficult to produce polyurethane with low batch to batch variability. This problem has been alleviated somewhat with a correlation between modulus and percentage of prepolymer in the mix. (See Figure 19.) By cutting a test strip from each casting, the material can be quickly evaluated by means of its modulus. Thus a uniform set of specimens can be gathered and variations due to slight modifications in the basic mix can be quickly detected. Scatter ranges are discussed under Material Characterization.

After curing was complete, the material was removed from the mold and placed in a dry box for two weeks or until used. The final product was a large round sheet 13 inches in diameter and $0.10^{\prime \prime}$ thick. When ready the smaller poker-chips were then cut into disks of approximately $2-1 / 2^{\prime \prime}$ in diameter. The resulting material specimen was virtually clear, which is one of the primary reasons for selection of S-113. It allows the possibility of either viewing directly, or photographing, the internal fracture process as it happens. This is a great advantage, for it is normally very difficult to surmise accurately what has happened during fracture solely from looking at the surface after the fact. Furthermore the material is optically very sensitive, and ideally suited for birefringence work. This property has been characterized and is alluded to in the discussion of material characterization. 
MATERIAL CHARACTERIZATION

The characterization of $\mathrm{S}-113$ has received the attention of several investigators including Williams, Ferguson, and Arenz (52) as well as Knauss, (53) and Zak. (54) These include various mechanical property definitions under infinitesimal strains, including a rather complete description of viscoelastic properties which even encompasses the optical properties of birefringence. Figures 20 to 22 give information relating to the constitutive properties of S-113 in the form of the Relaxation Modulus, the Master StressStrain curve and the accompanying shift factor for them. The data was obtained using standard techniques primarily based on the constant strain rate test. The relationship between the constant strain rate response and the relaxation modulus is straightforward and can be derived as follows. For a linear viscoelastic material the stress-strain law can be written in an integral form

$$
\sigma(t)=\int_{0}^{t} E(t-\tau) \frac{d \epsilon}{d \tau} d \tau
$$

where $\sigma$ is the stress, $E(t)$ is the relaxation modulus, $\epsilon$ the strain and $t$ the time. For constant strain rate conditions

$$
\epsilon=\mathrm{Rt}
$$

where $\mathrm{R}$ is the strain rate. Making an independent variable substitution under the integral sign of equation (3.1)

$$
\mathrm{x}=\mathrm{t}-\tau
$$




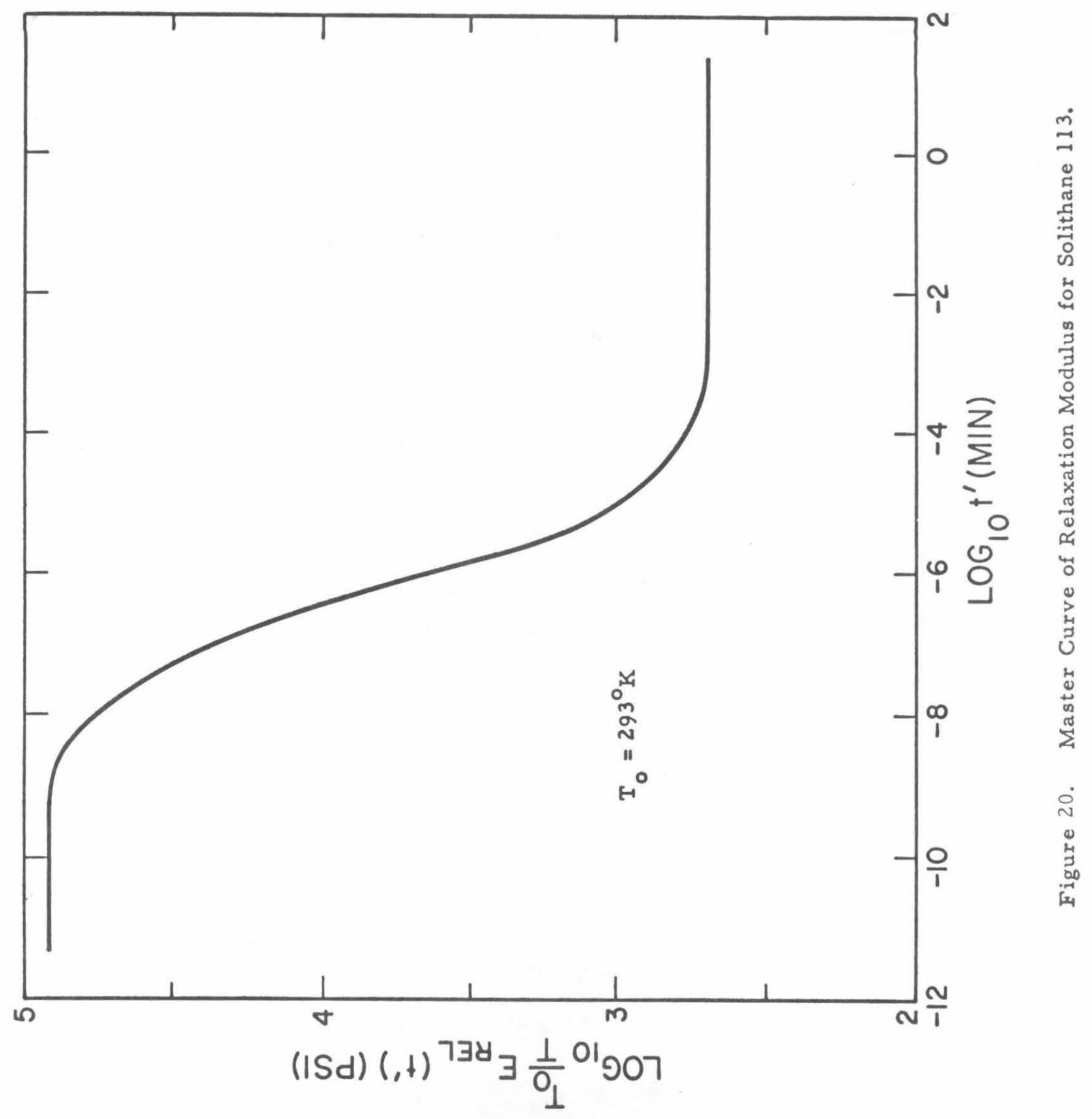




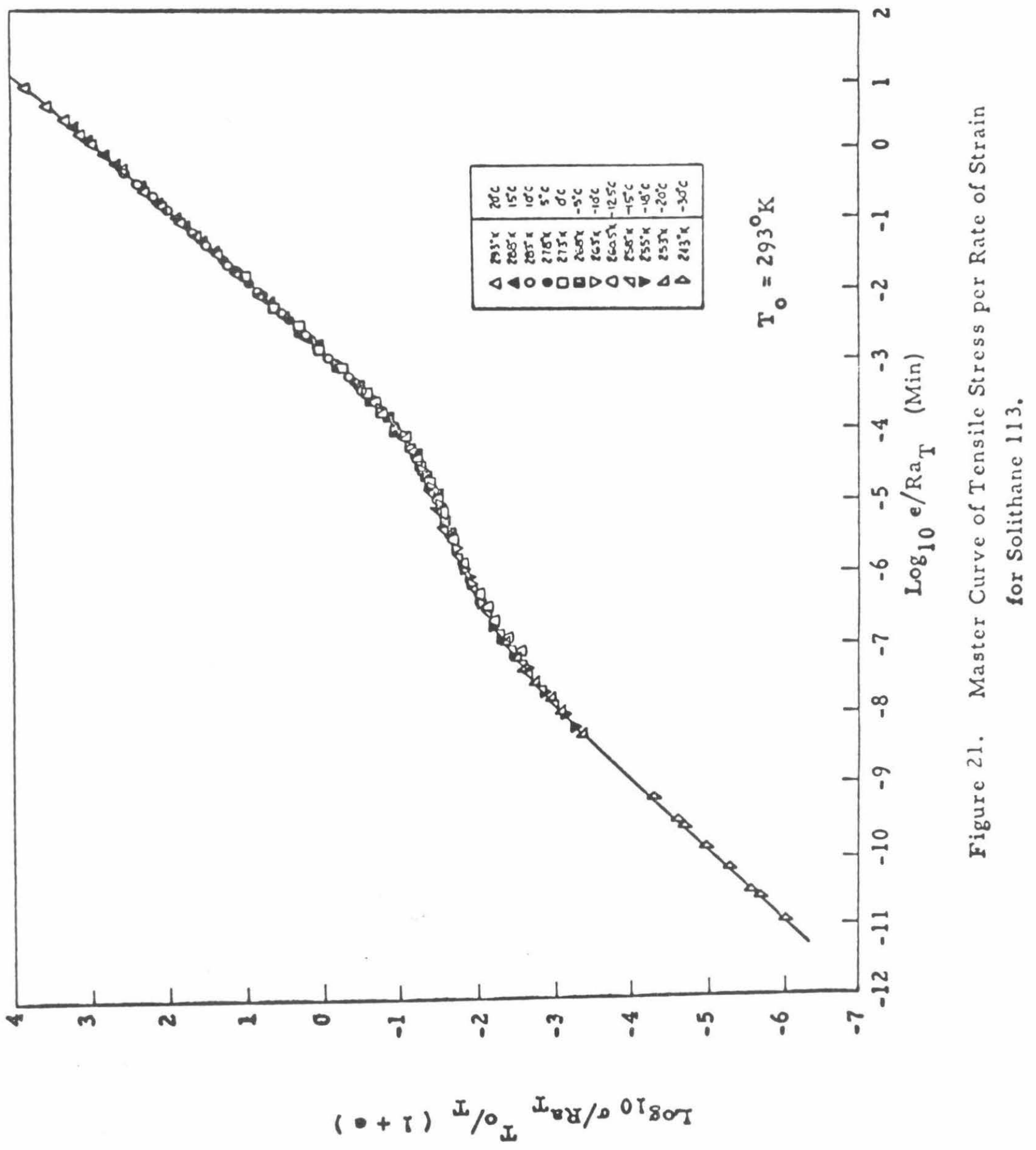




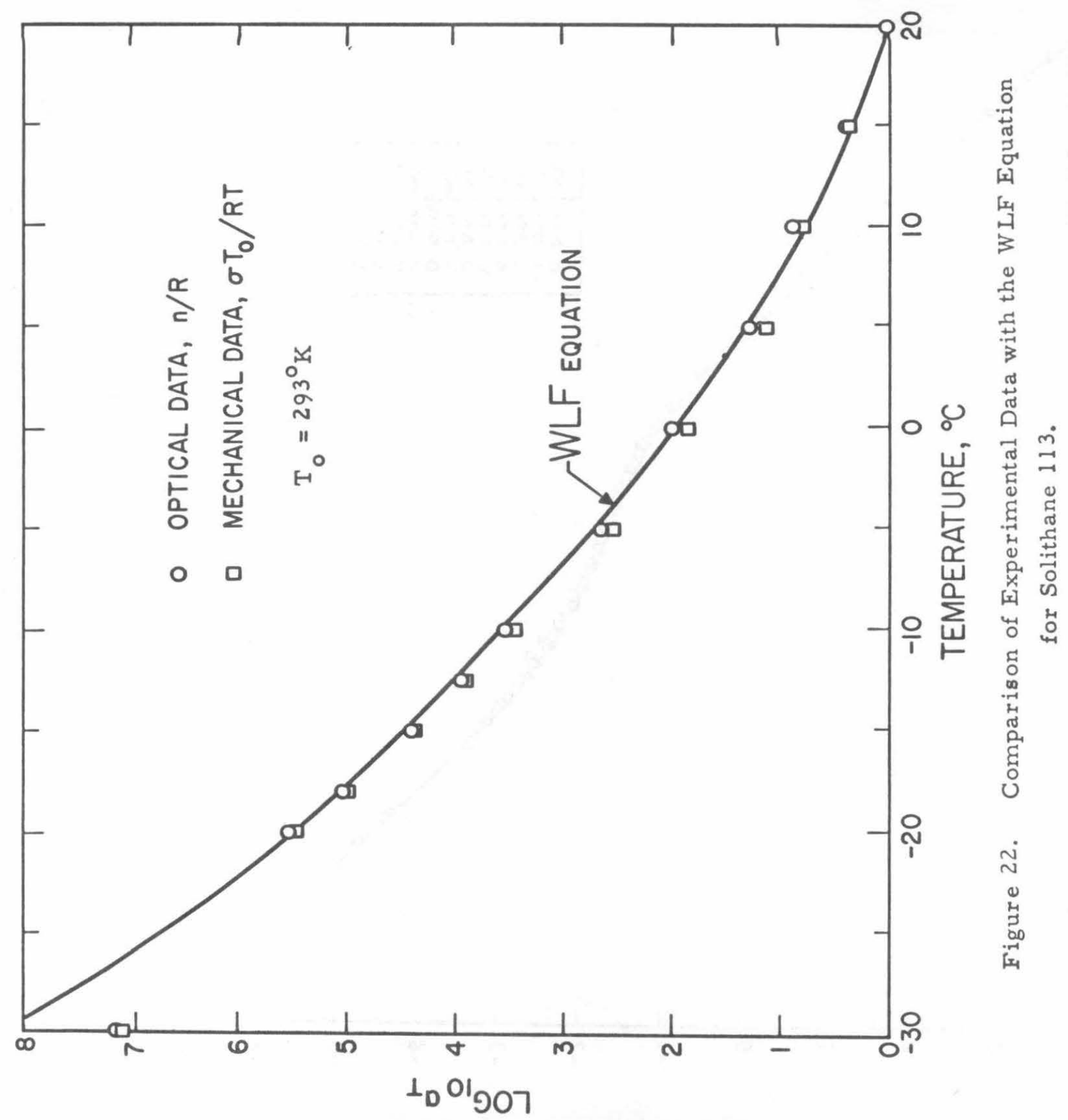




$$
\sigma(t)=-R \int_{0}^{t} E(x) d x
$$

From equation (3.4) it follows that

$$
-\frac{1}{R} \frac{d \sigma(t)}{d t}=E(t)
$$

Equation 3.5 shows that the relaxation modulus for a linear viscoelastic material is equal to the time rate of change of stress divided by the strain rate in a constant strain rate test. This relationship is used to evaluate the relaxation modulus from the data of the constant strain rate tests. The tests were performed for a wide range of rates and temperatures and then shifted (55) by the $\mathrm{a}_{\mathrm{T}}$ factor described in Figure 22 to give the composite master curves of Figures 20 and 21. The mechanics of data representation, interconversion, etc. is involved, but now reduced to standard practice as described in detail by Arenz, Ferguson, Kunio, Williams. (56) The curves indicate something of the nature of the material; however, the particular quantity to be noted at this time is the rubbery modulus of approximately $500 \mathrm{psi}$, which will be used in data reduction for the elastic analysis. Zak, (54) using an experimental technique suggested by Smith, (57) demonstrated that constant-strain rate tests at $0.02 \mathrm{in} / \mathrm{min}$. and $\mathrm{T}=25^{\circ} \mathrm{C}$, were in the rubbery regions for $\mathrm{S}-113$. This is the test condition used in the experiments to be described subsequently; consequently $\mathrm{E}=500 \mathrm{psi}$ is the pertinent material parameter. 
Bulk Properties

A side benefit of the poker-chip test is the measurement of the bulk mechanical properties that it provides through a knowledge of the apparent modulus. It will be recalled from equation (2.21) that if the normal stress in the axial direction $\sigma_{\mathrm{z}}$ is integrated over the face of the poker chip, the apparent modulus can be computed from the approximate stress analysis. This turns out to be expressed in terms of not only Young's modulus, but also the bulk modulus, which establishes a relationship between the three properties. This demonstrates that $\mathrm{E}_{\mathrm{A}}$ is an independent piece of information which can be used with the regular tensile modulus to define the two independent mechanical properties of an isotropic material. Figure 10 taken from Reference 41 graphically demonstrates this relationship and is a convenient tool for determining the bulk properties of materials. Thus armed with $\mathrm{E}$ and $\mathrm{E}_{\mathrm{A}}$, which turns out to be 15,400.psi, Bulk Modulus and Poisson's ratio can be determined for the material from Figure 10. For S-113, $\mathrm{K}=$ 47, 000 and $v=0.4978$. This value of Bulk Modulus may be somewhat surprising since it is actually of the order of one thousand times smaller than that of steel. It is only because the ratio of shear modulus to bulk modulus is so low that the incompressible assumption is justified. Of course, this ratio is also the reason for Poisson's Ratio being very nearly one half.

Finite Strain Characterization

Finite strain fields are commonplace in elastomers and 
require a special characterization for the material subjected to them. (4) Both uniaxial and biaxial tests have been conducted by Beckwith and Lindsey (58) to determine the form of the strain energy function, W. It is customary to express $\mathrm{W}$ as a function of the three strain invariants

$$
\begin{aligned}
& I_{1}=\lambda_{1}^{2}+\lambda_{2}^{2}+\lambda_{3}^{2} \\
& I_{2}=\lambda_{1}^{2} \lambda_{2}^{2}+\lambda_{1}^{2} \lambda_{3}^{2}+\lambda_{2}^{2} \lambda_{3}^{2} \\
& I_{3}=\lambda_{1}^{2} \lambda_{2}^{2} \lambda_{3}^{2}
\end{aligned}
$$

where $\lambda$ 's are stretch ratios. When the material is incompressible $\mathrm{I}_{3} \equiv 1$, and

$$
\mathrm{W}=\mathrm{W}\left(\mathrm{I}_{1}, \mathrm{I}_{2}\right)
$$

For the case of uniaxial tension $\lambda_{1}=\lambda$ and $\lambda_{2}=\lambda_{3}=\lambda^{-1 / 2}$, and the corresponding tensile stress is (4)

$$
\sigma=2\left(\lambda-\lambda^{-2}\right)\left(\frac{\partial \mathrm{W}}{\partial \mathrm{I}_{1}}+\frac{1}{\lambda} \frac{\partial \mathrm{W}}{\partial \mathrm{I}_{2}}\right)
$$

By dividing out the first factor and plotting it versus $1 / \lambda$ and using experimental data from a uniaxial strip, we obtain Figure 23. (The two sets of data points demonstrate maximum batch to batch variability.) From the trend of the data it can be inferred that the intercept, $\partial \mathrm{W} / \partial \mathrm{I}_{1}$, is constant and the slope, $\partial \mathrm{W} / \partial \mathrm{I}_{2}$, is zero. The strain energy function must then be of the form

$$
\mathrm{W}=\mathrm{C}_{1}\left(\mathrm{I}_{1}-3\right)
$$




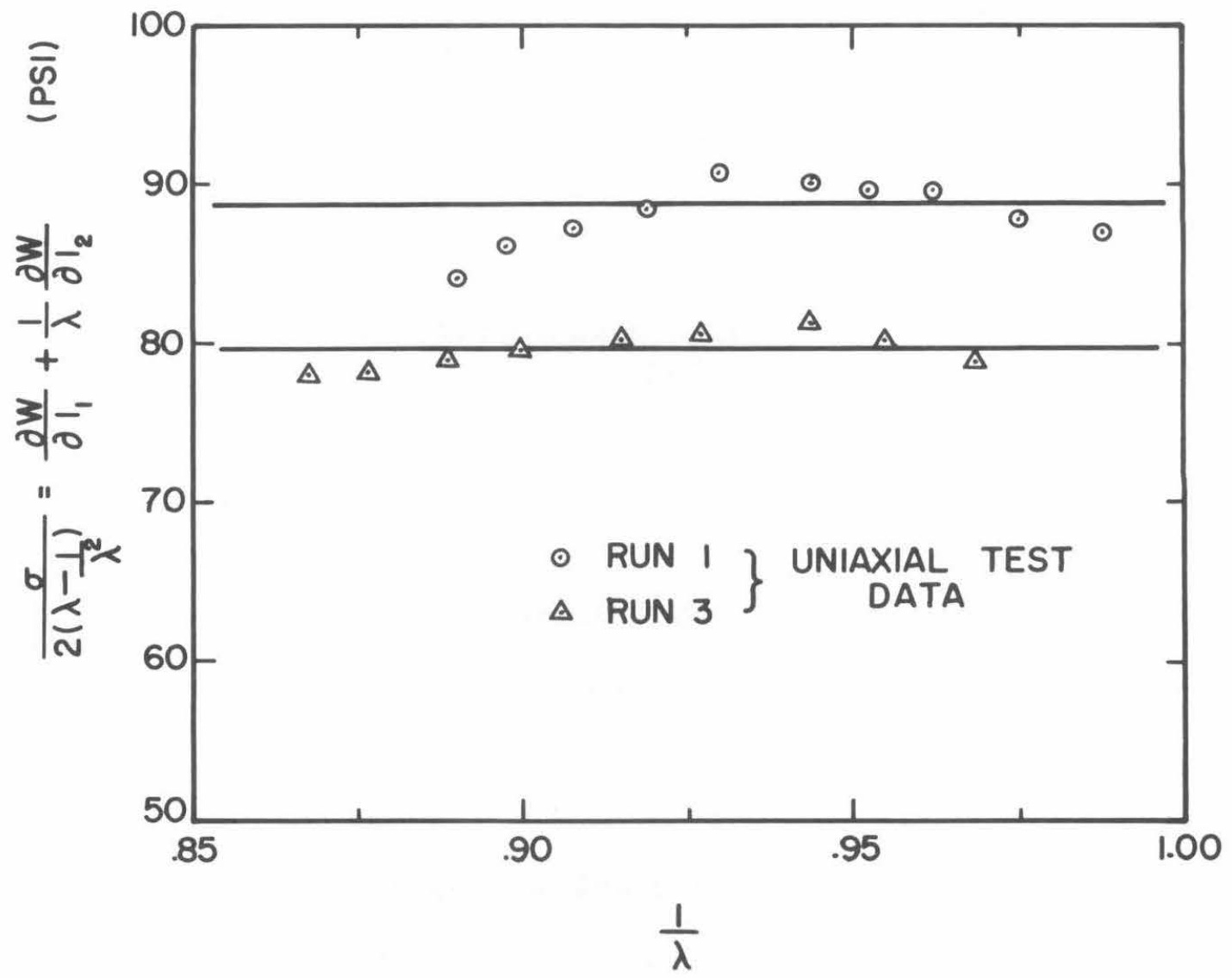

Figure 23. Dependence of Deformation State on Invariants $I_{1}$ and $\mathrm{I}_{2}$. 
where the factor three makes the function vanish in the unloaded state; i.e., $\lambda_{i}=1$. This classifies the finite strain behavior of S-113 as Neo-Hookean, which is the simplest representation and a mark in its favor. Simplicity in analysis is a big asset in large deformation problems.

Now to see exactly how close S-113 follows Neo-Hookean behavior in uniaxial tension, Figure 24 has been plotted. Only in the larger values of stretch ratio near fracture is there any perceptible deviation. Also the value of the material constants agree well with the infinitesimal limit case, which defines $C_{1}=E / 6$.

Corroboration of the Neo-Hookean result based on uniaxial test was made from equal biaxial tests on the test rig pictured in Figure 4. In this case $\lambda_{1}=\lambda_{2}=\lambda$ and $\lambda_{3}=\lambda^{-2}$ from the incompressibility condition. The corresponding in-plane stress turns out to be (4)

$$
\sigma=\frac{\mathrm{pR}}{2 \mathrm{t}}=2\left(1-\lambda^{-6}\right)\left(\frac{\partial \mathrm{W}}{\partial \mathrm{I}_{1}}+\lambda^{2} \frac{\partial \mathrm{W}}{\partial \mathrm{I}_{2}}\right)
$$

where $\mathrm{p}=$ internal applied pressure

$$
\begin{aligned}
& \mathrm{R}=\text { radius of curvature } \\
& \mathrm{t}=\text { sheet thickness }
\end{aligned}
$$

Proceeding upon the assumption that S-113 is Neo-Hookean, equation (3.9) becomes

$$
\frac{\mathrm{pR}}{4 \mathrm{t}}=\mathrm{C}_{1}\left(1-\lambda^{-6}\right)
$$

Assuming a circular arc deflection curve, the radius of curvature for a sheet of original radius a and pole height $h$ (see Figure 4) is 


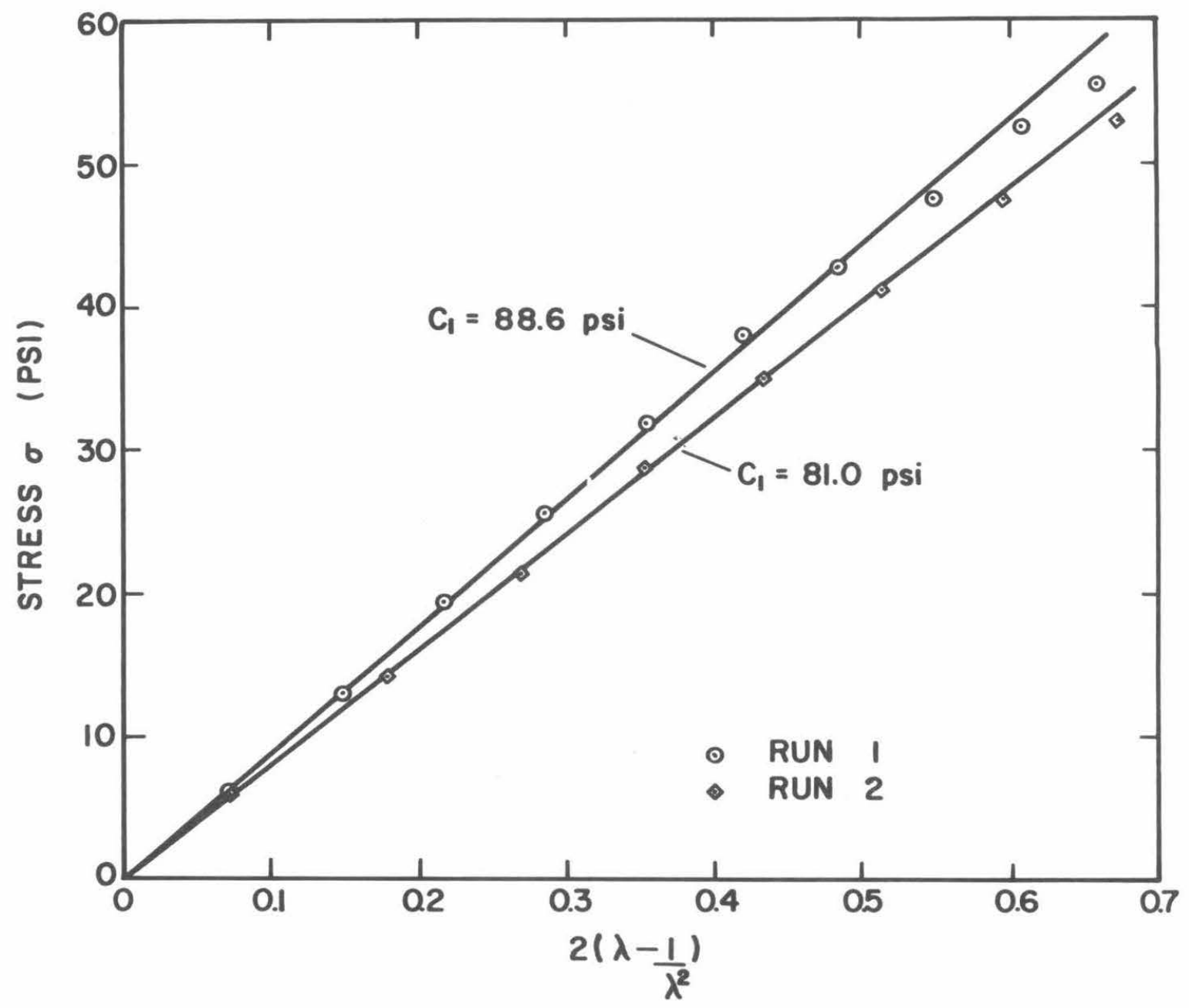

Figure 24. Correlation of Neo-Hookean Representation with Uniaxial Experimental Tests. 


$$
\mathrm{R}=\frac{\mathrm{a}^{2}+\mathrm{h}^{2}}{2 \mathrm{~h}}
$$

Giving

$$
\frac{\mathrm{p}\left(\mathrm{a}^{2}+\mathrm{h}^{2}\right)}{8 \mathrm{ht}}=\mathrm{C}_{1}\left(1-\lambda^{-6}\right)
$$

By measuring $p, h$ and $\lambda$ it was possible to construct Figure 25 for an initial sheet radius of five inches. The plot indicates a linear relation except in the initial stages of deformation. It was definitely established that the nonlinearity was due to slippage of the sheet underneath the clamping ring making the measured value of $\mathrm{h}$ too large. The ring is not fastened down too tightly since premature fracture at the grip will ruin the test. After the initial slippage the curve then becomes linear to establish Neo-Hookean behavior.

\section{EXPERIMENTAL APPARATUS FOR THE POKER-CHIP TEST}

As the equations and figures of Chapter II demonstrate, the poker-chip test constitutes a vehicle by which a hydrostatic tensile field can be applied to a local region of a body whose actual dimension is dictated by experimental practicality. The theoretical analysis shows that an aspect ratio of fifteen to one is the lower limit required to produce this stress field and twenty to one is more desirable. A two-inch diameter with 0.10 inch thickness was selected to be a reasonable compromise between what could be effectively bonded and pulled and what was desired in the way of observation. Although Gent and Lindley originally performed the poker-chip test by pulling or squeezing rubber blocks between 


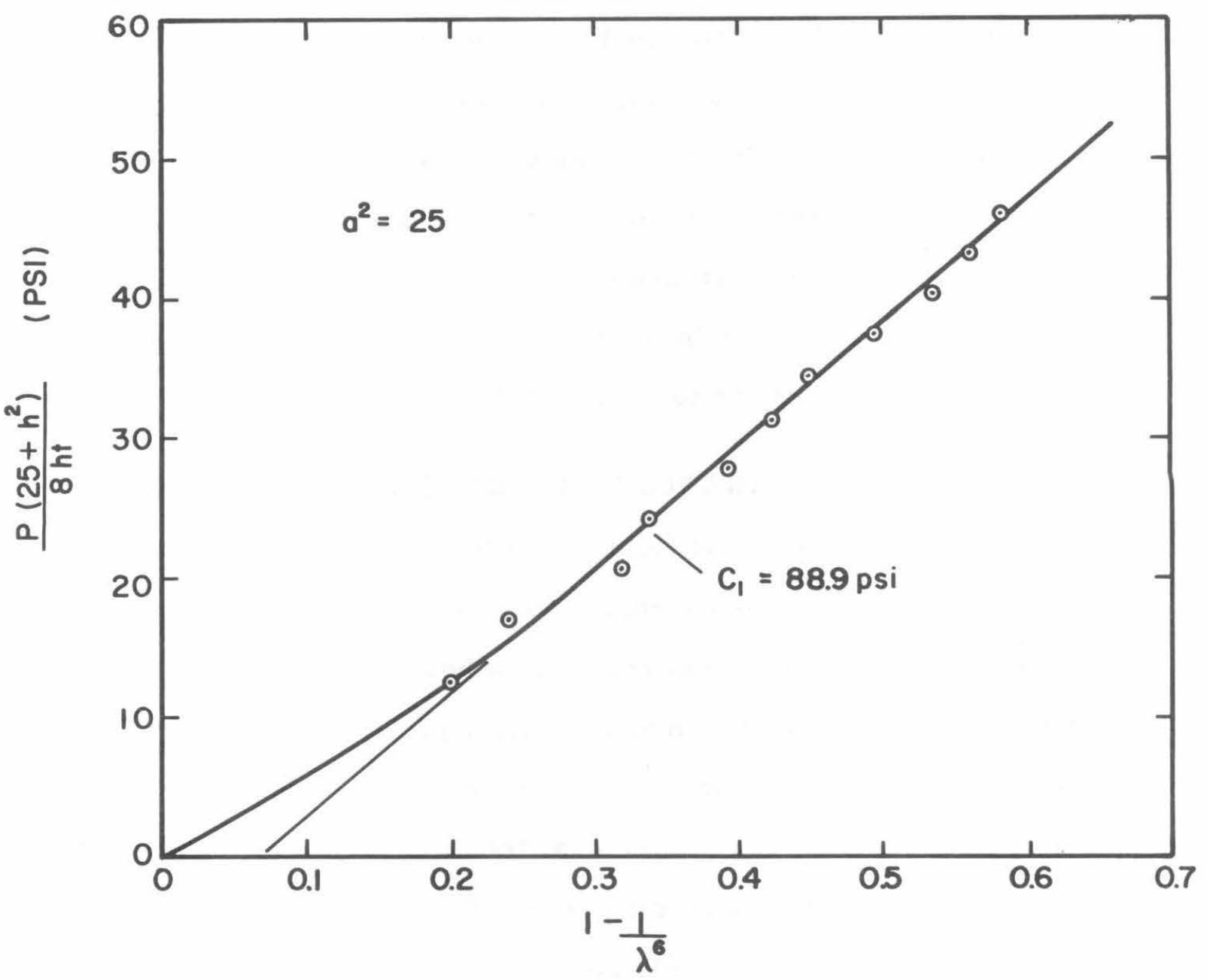

Figure 25. Correlation of Neo-Hookean Representation with Biaxial Experimental Tests. 
steel plates, it was decided for the experimental work on S-113 that the apparatus would be altered considerably to facilitate a more thorough study of the actual mechanisms of the fracture process. This was accomplished by using transparent lucite grips in place of steel. Lucite has a modulus in the neighborhood of 500,000 psi (59) and this provides approximately a 1,000 to 1 ratio between modulus of grip and specimen, which closely duplicates the theoretical assumption of rigid boundaries made in the stress analysis. Coupled with the clear grip and clear specimen a transparent bonding agent was found in the form of Eastman 910 , which happily acted as a wetting agent for both the rubber specimen and the lucite grip. When the assembly was complete, a window was produced through which the observer could view the internal regions of the material and study the fracture process. A schematic drawing of the entire assembly is shown in Figure 26 where the arrows indicate the attachment of a testing machine through which the specimen was loaded.

\section{Bonding Procedure}

A very critical facet of the experimental technique of the poker-chip test is the bonding procedure. Being somewhat of an art, it is demanding upon the technician to produce a bond with sufficient strength that the specimen will not tear away from the grip before an internal fracture is produced. The specimen is prepared by roughly cutting it to size and then cleaning it thoroughly with a cloth slightly dampened in methyl alcohol. If used sparingly this procedure will not produce noticeable swelling in 


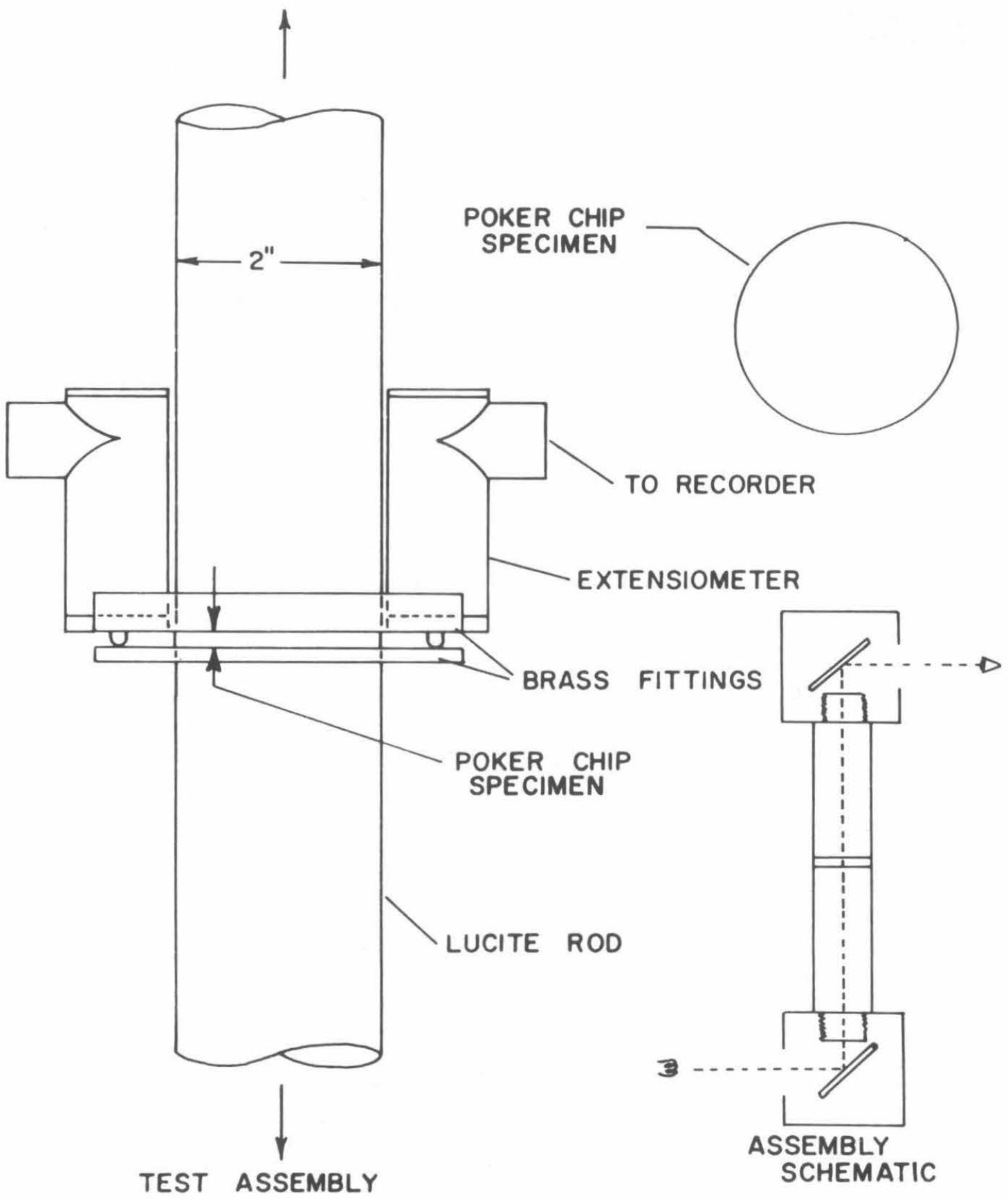

Figure 26. Schematic of Poker-Chip Test Apparatus. 
the rubber. The lucite grips must be lightly roughened and thoroughly cleaned; furthermore it was found advisable to avoid silicon compound abrasives, and aluminum oxide paper was found to work nicely. A bonding accelerator (number GA-1A1) supplied by Eastman was then applied to the specimen and the Eastman 910 to the grip. The two pieces are brought together under hand pressure and allowed to set. The process is repeated for the other grip; however a V-block is used to insure perfect alignment. Absolutely no mis alignment can be tolerated due to the bending imposed in the test. The same is true of taper in the specimen; once bending is present, the local stress conditions become unknown and the test is of little use.

Optics

With the specimen bonded to the lucite, the grips were screwed into special fixtures depicted in Figure 26. Front surface mirrors were mounted at $45^{\circ}$ to the horizontal in these heads, providing a periscope arrangement for viewing the specimen. Through one mirror the specimen was illuminated by means of a Peck model 110 Mercury vapor point source and viewing or photographing took place through the other. With this powerful light source, it is possible to take high speed motion pictures of the fracture process to document a detailed history of it.

This method has also been successfully used even on quite opaque and translucent materials. These are not as desirable 
as the clear materials, nor are they as readily photographable, but fracture initiation is easily detected along with rather gross details of the propagation phase, depending upon the degree of opaqueness of the material. Furthermore, if the material being tested lends itself at all to the transmission of light, the fracture point can be detected, for there are instances recorded where it has been found difficult to ascertain the point at which the specimen actually failed because no direct manifestation of it was made on the load history. (60)

Strain Measurements

One of the many interesting aspects of this test is the fact that the overall specimen strain based upon the initial thickness is small; i.e., S-113, which evinces 35 percent to 40 percent ultimate strain in uniaxial tension, exhibits only two percent to three percent ultimate strain in hydrostatic tension. This was another reason for putting faith in the results of the approximate stress analysis which employed tools of infinitesimal elasticity. It should be pointed out that this configuration is one of the few wherein infinitesimal theory can be applied to fracture analysis of rubbery materials with confidence in the accuracy of the solution. Such a state of affairs is certainly welcome since use of finite elastic techniques for analysis is very limited and virtually never used in fracture studies; however, Levinson and Blatz (61) have employed a variational scheme to solve the poker-chip problem for large deformations, so that materials demonstrating strain levels out of the range of the linear 
theory or on the border of linear theory can still be analyzed for the poker-chip test.

Because the displacements were small, for instance in the tests to be discussed total displacement at the grip was in the neighborhood of 0.002 inches, extensometers were used to measure the axial separation of the lucite grips. (See Figure 26.) They incorporated linear, variable, differential transformers, LVDT, in conjunction with Schaevitz LVDT exciter, demodulator, type DMPS-3. These operated into a Moseley Autograf, X-Y Recorder, Model 2FR-A(S), made in Pasadena, California, where the displacements were magnified 2000 times. A photograph of the actual specimen with the LVDT's mounted in place is shown in Figure 27. The complete assembly was placed in a Universal Testing Machine, Model Number TTC, manufactured by Instron Engineering Corporation of Canton, Massachusetts. A photograph of the apparatus ready for test is given in Figure 28.

\section{EXPERIMENTAL RESULTS}

With a knowledge of the two independent mechanical properties $v$ and E, Figure 8 can be used to find the relationship between the internal field stress in the poker-chip and the externally applied strain $\epsilon_{1}$, which of course can in turn be related through the apparent modulus to the applied load. The load was correlated with the displacement through an apparent stress-strain curve. This was computed by considering the specimen to be a uniaxial tensile bar where the stress is obtained by dividing the applied load by the 


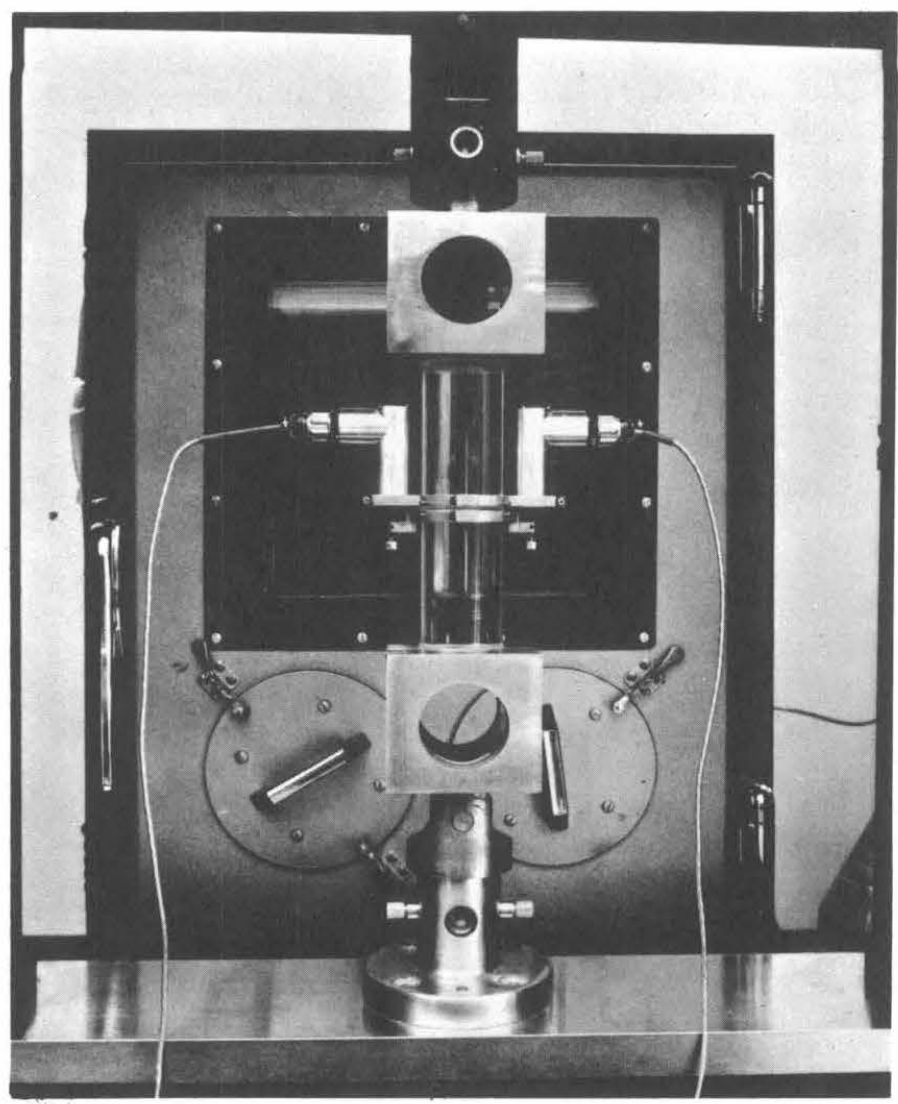

Figure 27. Arrangement of the Triaxial Test. 


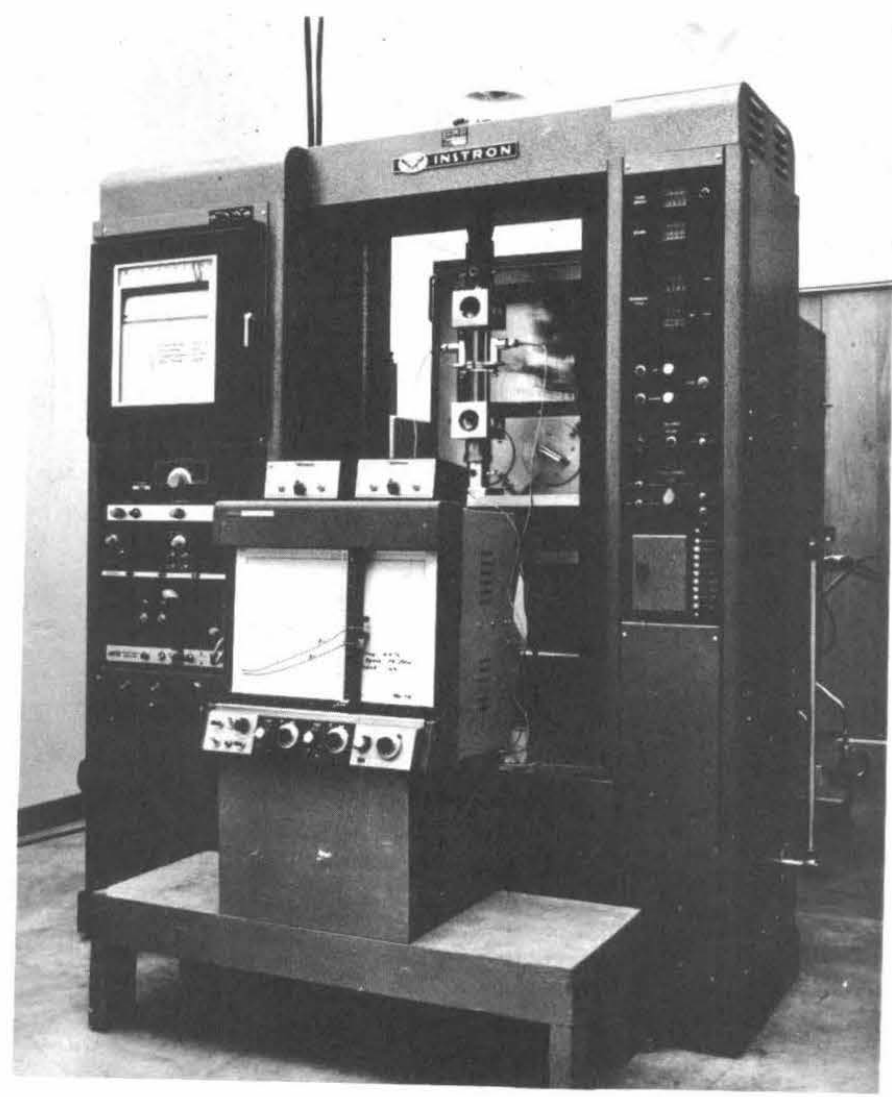

Figure 28. General View of the Triaxial Test Showing the Specimens in the Instron Testing Machine and the Recording Equipment. 
cross-sectional area of a large face, P/A, while the strain is computed like a tensile bar on the basis of specimen thickness. The descriptive parameter for this pseudo stress-strain curve is the apparent modulus, $E_{A}$, computed in the obvious way by dividing the equivalent uniaxial stress by the equivalent uniaxial strain. A plot of several typical apparent stress-strain curves obtained at a strain rate of $0.02 \mathrm{in} / \mathrm{min}$, is given in Figure 29. showing the rubbery, elastic response of $\mathrm{S}-113$ in $\mathrm{HT}$. This gives some indication of the scatter in the apparent modulus due to the batch to batch variability of the material. Both the mode and the average come out to be approximately 15,400 psi with a maximum variation of \pm 8 percent.

As can be seen, the apparent stress-strain relation is linear well above one percent strain, and then it bends over as it proceeds to fracture at strains of $2-1 / 2$ percent to 3 percent. The actual fracture stress levels involved are considerably higher than their uniaxial counterparts; however as was discussed previously, the ultimate strain is greatly reduced. From Figure $8, \sigma_{\mathrm{z}} / \mathrm{E} \epsilon=48$, which gives a local HT of 625 psi. S-113 possesses an ultimate strength in uniaxial tension of approximately 160-200 psi, (62) while local stress values in the hydrostatic configuration range approximately three times this value in the neighborhood of 500-650 psi. These values quoted represent the range of a large number of tests conducted at room temperature.

Stress-Axis Theorem

It is interesting to abstract from this and other work (22) 


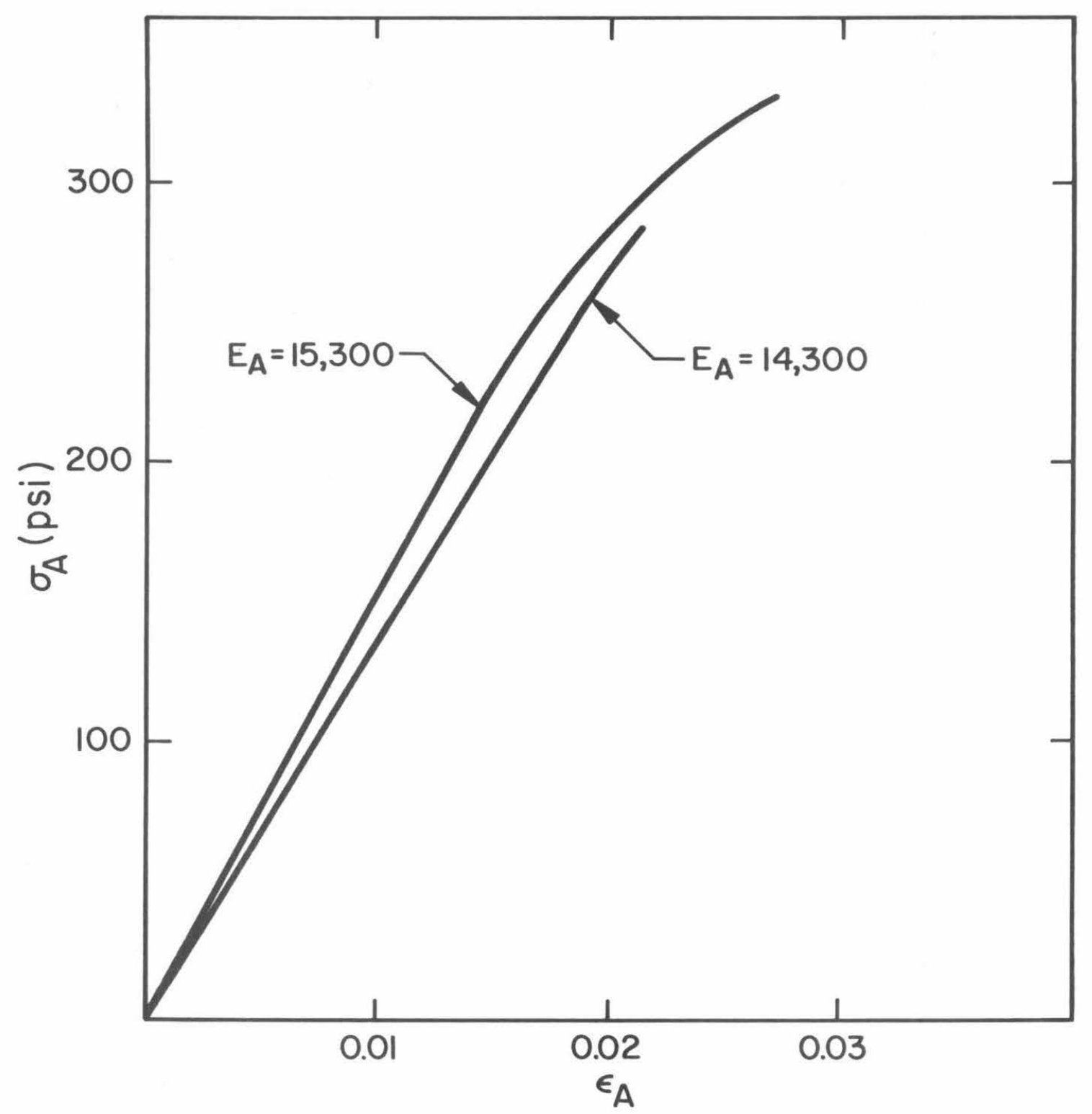

Figure 29. Apparent Stress-Strain Curve for Poker Chip Test. 
a theorem on fracture, which can be conceived to provide a guide for engineering intuition. It can be stated roughly that as the number of axes of stress increase the ultimate stress is increased and the ultimate strain is decreased; furthermore, as the degree of axiality of stress is altered toward the hydrostatic this same trend will be emphasized. In other words, as an additional axis of stress is added to a body in uniaxial tension, the ultimate stress and strain increase and decrease respectively from the uniaxial value as the new stress component grows from zero to become equal to the original one producing a state of equal biaxial tension. Adding another axis of stress to the biaxial case further alters the ultimate properties in the same direction as it grows from zero to become equal to the original two and produce a state of three-dimensional hydrostatic tension. Consequently the state of hydrostatic tension is a limit case in that it provides the maximum in stress that a given material can withstand, as well as a minimum in strain at which fracture can be produced. In essence the material performs at its best in this geometrical configuration if dilatation is the criterion; however if strain is the criterion, pure distortion states are by far superior.

\section{Description of Fracture}

The actual physics of the fracture process are quite unusual and produce a unique mode of rupture. As the load is applied, the field remains seemingly undisturbed even while observing the specimen through crossed polaroids. S-113 is a very sensitive birefringent material; in fact this is one of its most outstanding 
properties, and yet no fringes are seen to appear in the field until the fracture process begins. (This constitutes additional evidence to the condition of HT alluded to previously. ${ }^{*}$ ) Then at an average applied stress of approximately 300-350 psi or a local field stress of 500-650 psi, what appears to be a bubble catastrophically bursts into the field somewhere in the vicinity of the center of the specimen. It rapidly grows in a spherically radial fashion resembling an ablating cavity until it fills the entire thickness of the specimen and interacts with the boundary. Figure 30 is a composite photograph depicting this growth which requires about 0.016 seconds. The data was obtained with a $16 \mathrm{~mm}$, Beckman and Whitley, Magnifax High Speed Camera $(63 \mathrm{~mm}, \mathrm{f} / 2 \mathrm{lens})$ at a framing rate of 2000 frames per second. Next, two of the extremities of the cavity tail off into a sharp crack that appears to propagate perpendicular to the plane of maximum principal stress. Subsequently one and occasionally two such nuclei manifest themselves and the specimen appears much like the sketch in Figure 31 below.

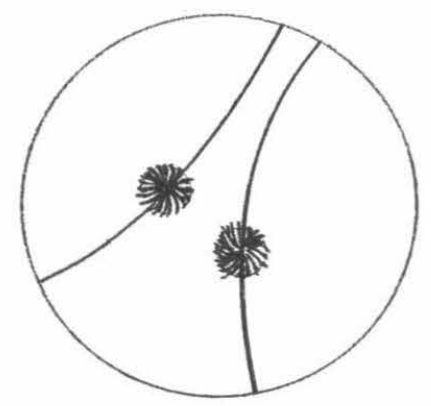

Fig. 30. Axial view of poker-chip fracture field.

* The number of fringes is proportional to the difference in principal stress, $n \approx \sigma_{1}-\sigma_{2}$. 
$-86-$
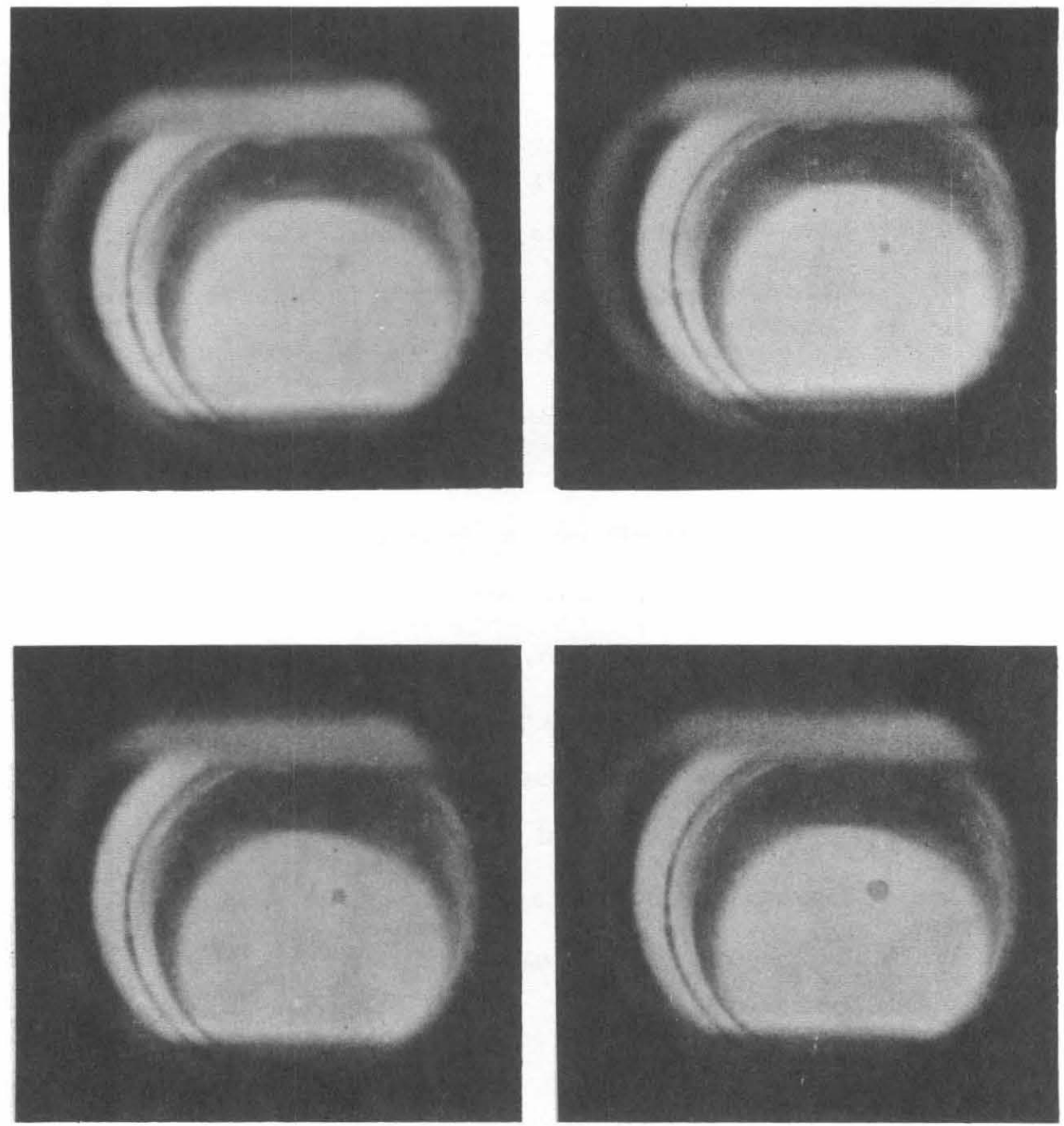

Figure 31: Internal Fracture Growth History 
Fracture Surface

If the specimen is further loaded beyond the initiation point until it is actually pulled completely apart into two pieces, the resulting mating surfaces are completely riddled with crevices and fissures and appear badly mottled. Figure 32 is a photograph of a typical poker-chip fracture where the points of initiation can be readily discerned with some experience. One of the facets of fracture in this geometry that makes it unique is the location of the point of initiation of fracture. In all other test specimens the fracture initiates at a manufactured boundary, and in some degree the ultimate properties are actually a measure of how well that surface is prepared. In this case, however, the fracture originates wholely within the material. It is a true test of the material strength, which is determined by the structure of the material and by the existence of internal flaws. By focussing in closer on the origin of fracture, a nucleus is discovered as photographed in Figure 33, where the magnification is $100 \mathrm{X}$. The surface as seen here is inclined at an angle of approximately $20^{\circ}$ to $30^{\circ}$ and is consequently distorted slightly, but the floral pattern emanating from the nucleus shows the rather regular manner in which the crack propagates radially away from the original nucleus or cavity. The surface in focus here is virtually planar and consequently what had appeared to be a bubble growing in the field while the fracture was being observed is believed to have actually been a circular, planar, Saturn-ring crack that was enlarging in a radial direction, but due to the hydrostatic stress field, its mating surfaces were pulled apart and made to 
$-88-$

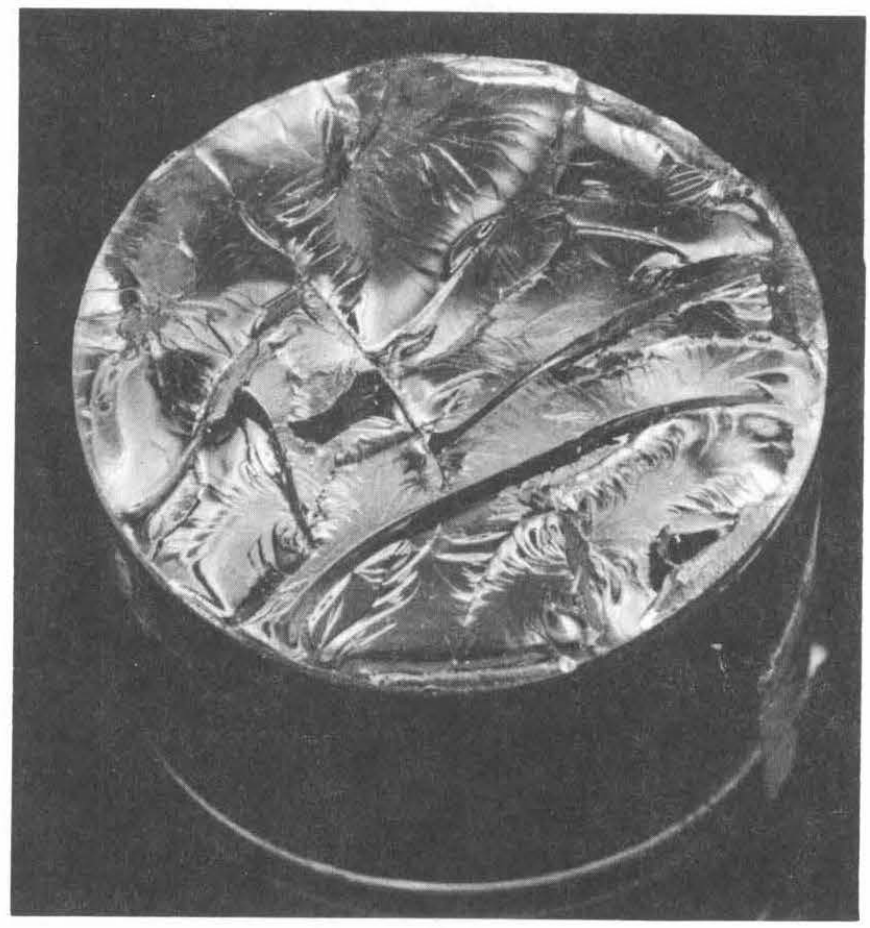

Figure 32: Fracture Surface of Poker Chip Specimen

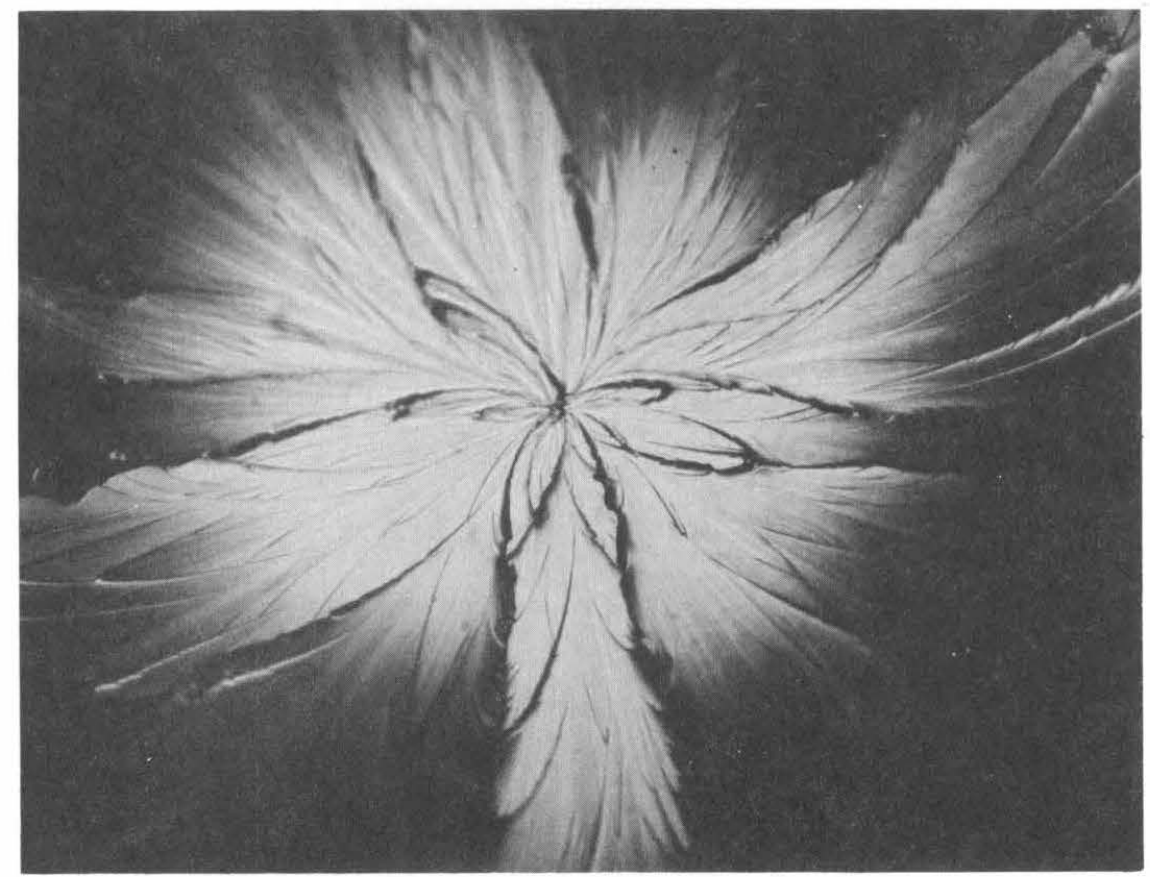

Figure 33: Nucleus of Fracture 100X 
$-88 a-$

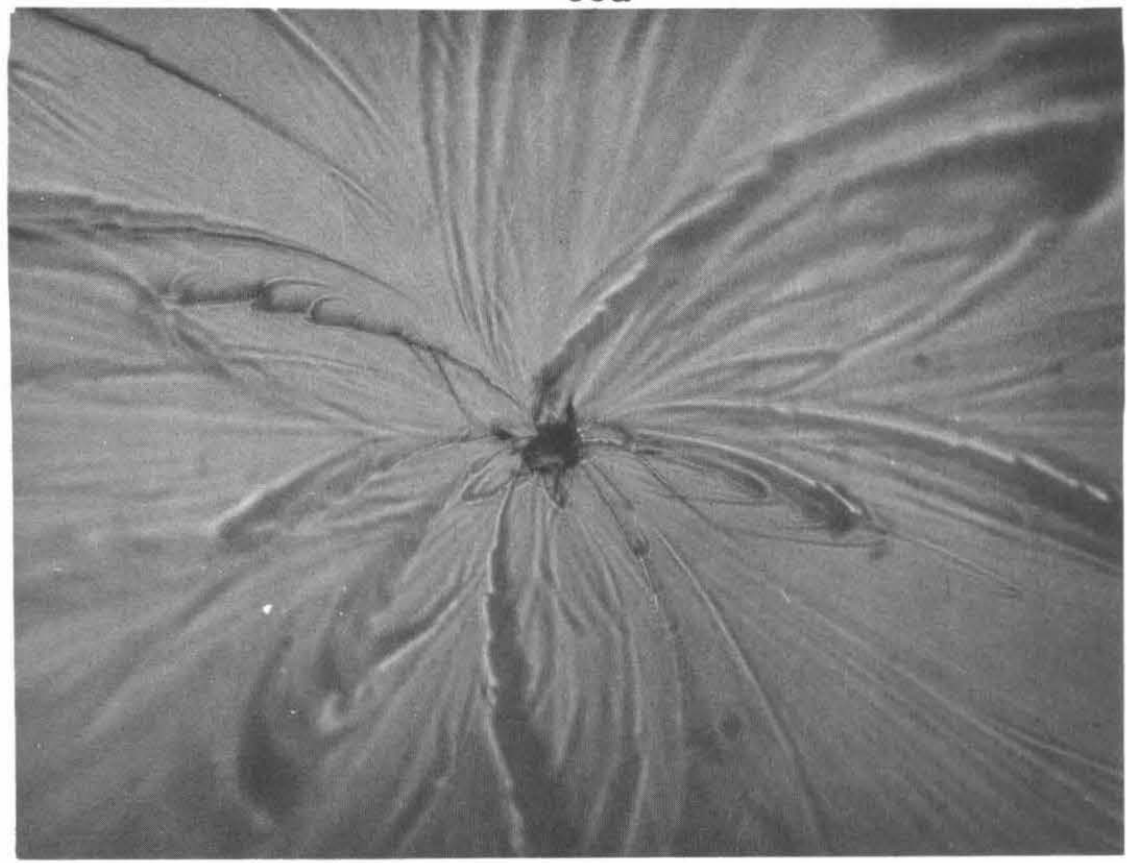

Figure 34: Nucleus of Fracture 200X

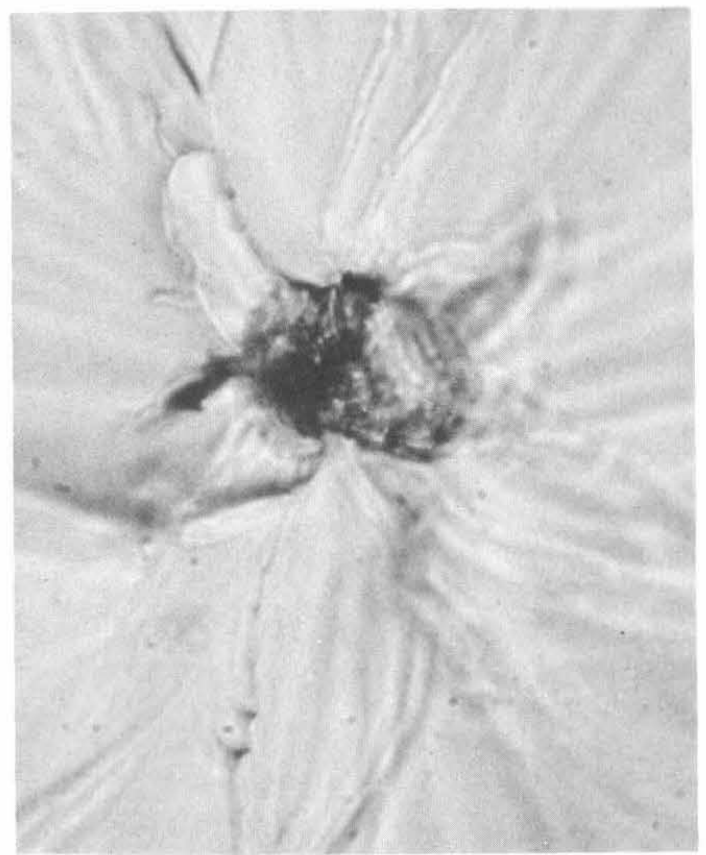

Figure 35: Nucleus of Fracture $800 \mathrm{X}$ 
resemble a spherical bubble that was ablating. This interpretation is further substantiated by unloading the specimen and watching the collapse of the bubbles back into the flat planar cracks. The crack surface is not perfectly planar but does possess a slight curvature which seems to become more pronounced as the nucleus moves closer to one of the boundaries. The orientation of this pseudoplane seems to be reasonably random, but if there is any bias, it is toward planes tending to the vertical.

A more highly magnified view of the origin of fracture shows a very rough and cobbled area as seen in Figures 34 and 35 which was, in these specimens, consistently found to be of the order of $10^{-3}$ inches in diameter. The region outside of the dark center circle is a glassy, mirror-like surface characteristic of the high velocity phase of propagation. Since the pattern and dimension of the striations radiating from the nucleus were so reproducible, they must be evidence of some fundamental mechanism transpiring in fracture. Knauss (63) has discussed the notable difference in appearance in fracture surfaces of an 'H.C.' rubber, (64) and has been able to draw quantitative correlations between velocity of propagation and roughness of surface. The two materials bear enough similarity that it should be possible to make inferences about rate of propagation in S-113. Based on Knauss' observations, the cobbled surface around the nucleus would have been formed from a very slowly moving crack--almost of zero velocity. Fracture then would resemble severing bonds almost individually like rubber bands. The glassy surface on the other hand is a sign of very high velocity 
motion, which would correspond with the short times noted from the high-speed films. The change in surface is quite abrupt, indicating a very rapid change from the slow to the fast mode. These observations would then support the hypothesis that fracture begins from a nucleus that is formed very slowly in the material. It might even be considered as a flaw that is "grown' in the material under load, which, upon reaching a certain critical size, triggers a catastrophic rupture at high velocities.

Fracture Propagation

High speed motion pictures (1000 to 3000 frames per second) of the fracture process taken through the periscopic mirrors have documented portions of the propagation behavior. Unfortunately they did not shed light on the initiation mechanisms, but the character of propagation in the high velocity mode is documented. Figure 36 shows the short period of acceleration from the zero velocity region to some critical velocity which remains essentially constant with some evidence of a stick-slip behavior. The data for these curves were obtained by photographing the growth of the fracture surface that was mentioned previously as having the appearance of a bubble, but which is actually a planar crack that has been pulled apart by the HT. The test was conducted at room temperature at a strain rate of $0.20 \mathrm{in} / \mathrm{min}$. The films were subsequently projected and measurements made of the diameter of the circular crack as a function of time obtained through a knowledge of the framing speed, which was calculated through timing blips made on the film. Due to the limitations set by the grain of the film and the magnification 
$-91-$

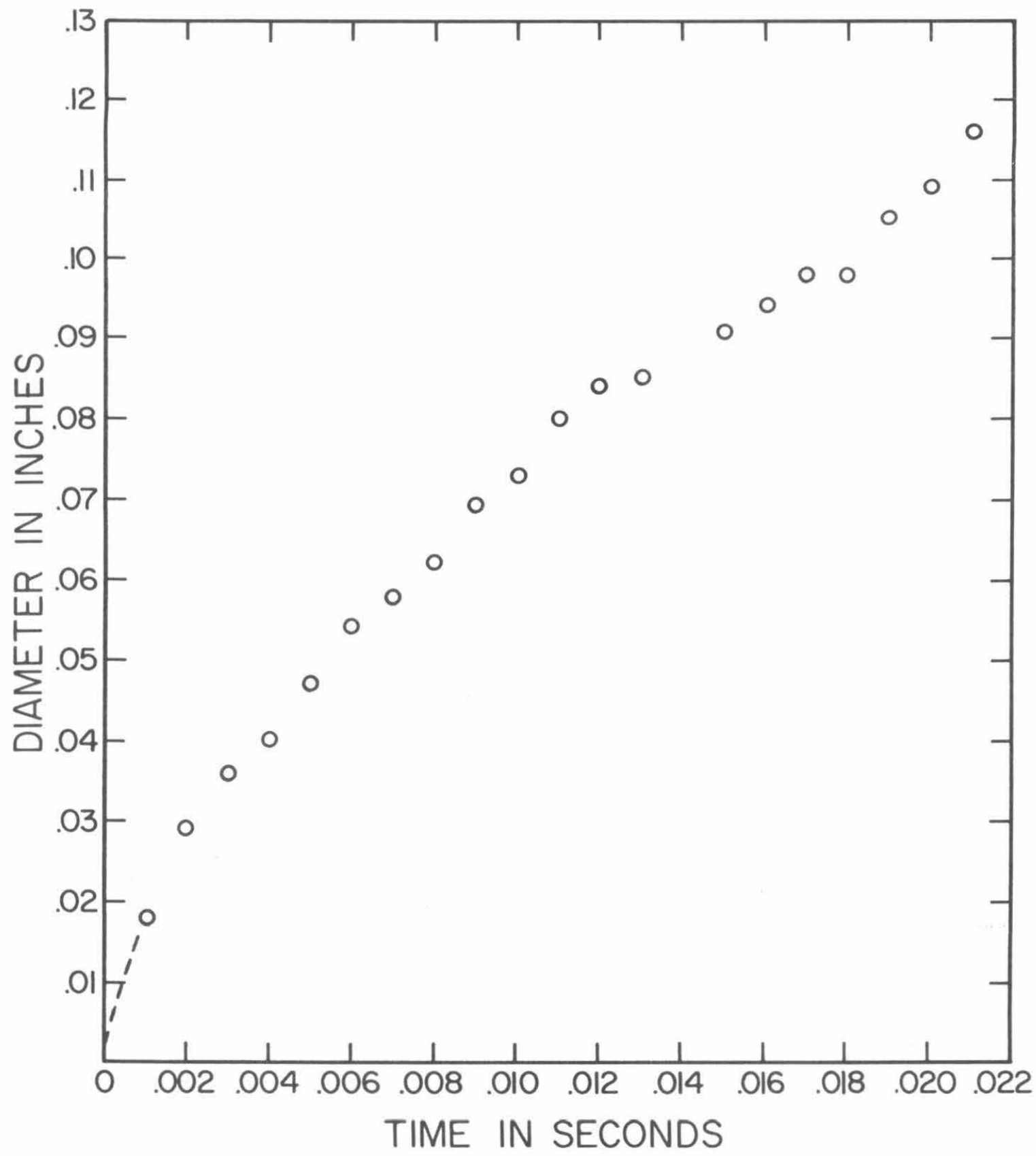

Figure 36. Growth Rate of Saturn Ring Crack Strain Rate 0.20 in $/ \mathrm{min}$. 
under which the actual filming was performed, the entire propagation history could not be recorded; especially the short-time regime. This is indicated in the figure by a dotted line as an extrapolation back to the original flaw size.

It appears then in retrospect from evidence studied a posteriori and coupled with other evidence acquired before and during fracture that:

1) Fracture nucleates probably from a point of weakness in the material.

2) The early phase of fracture is a static one that can be approximated as crack propagation at virtually zero velocity, where bonds are broken slowly.

3) After reaching a critical size the crack will rapidly accelerate and the remaining surface changes from a rough and coarse one to a mirror-like or glassy one.

4) This critical stress is almost three times the uniaxial strength.

5) The acceleration period is very short and is followed by a steady state situation where the fracture surface propagates at a constant velocity until it intersects the boundaries.

6) It then propagates as a very blunt crack along a trajectory that takes it perpendicular to the plane of the maximum principal stress of the distorted field. 


\section{FLAW DESCRIPTION}

In the description of material fabrication covered in Chapter III, it was mentioned that the mix was degassed under vacuum to pull out entrapped gases dissolved in the components. Furthermore if there were any trace of water present, a side reaction would result, and carbon dioxide would be given off, continuing as long as any water was available. Consequently the probability is quite high that miniature flaws are produced in the material in the form of entrapped gaseous bubbles. Although a large volume of gas is removed by vacuum, there seems to be a limit to the size of bubbles that can be drawn out of such a viscous liquid. Visual inspection of a cast sample will not detect any indication of bubble-type flaws; however microscopic observation at $200 \mathrm{X}$ has proved their existence, as shown in the photograph of Figure 37.

In fact such flaws have been found in quantity. Samplings taken at random throughout the castings, which in our particular specimens are in the form of circular sheets $13^{\prime \prime}$ in diameter and $0.10^{\prime \prime}$ thick, show uniformity from section to section both in size and distribution of the voids. By and large the voids ranged from 3 to 5 times $10^{-4}$ inches in diameter with the larger or smaller voids occurring only in exceptional cases. Figure 38 shows a typical mapping of a section of a sheet with a typical ratio of void volume to material volume. A representative figure for this ratio 

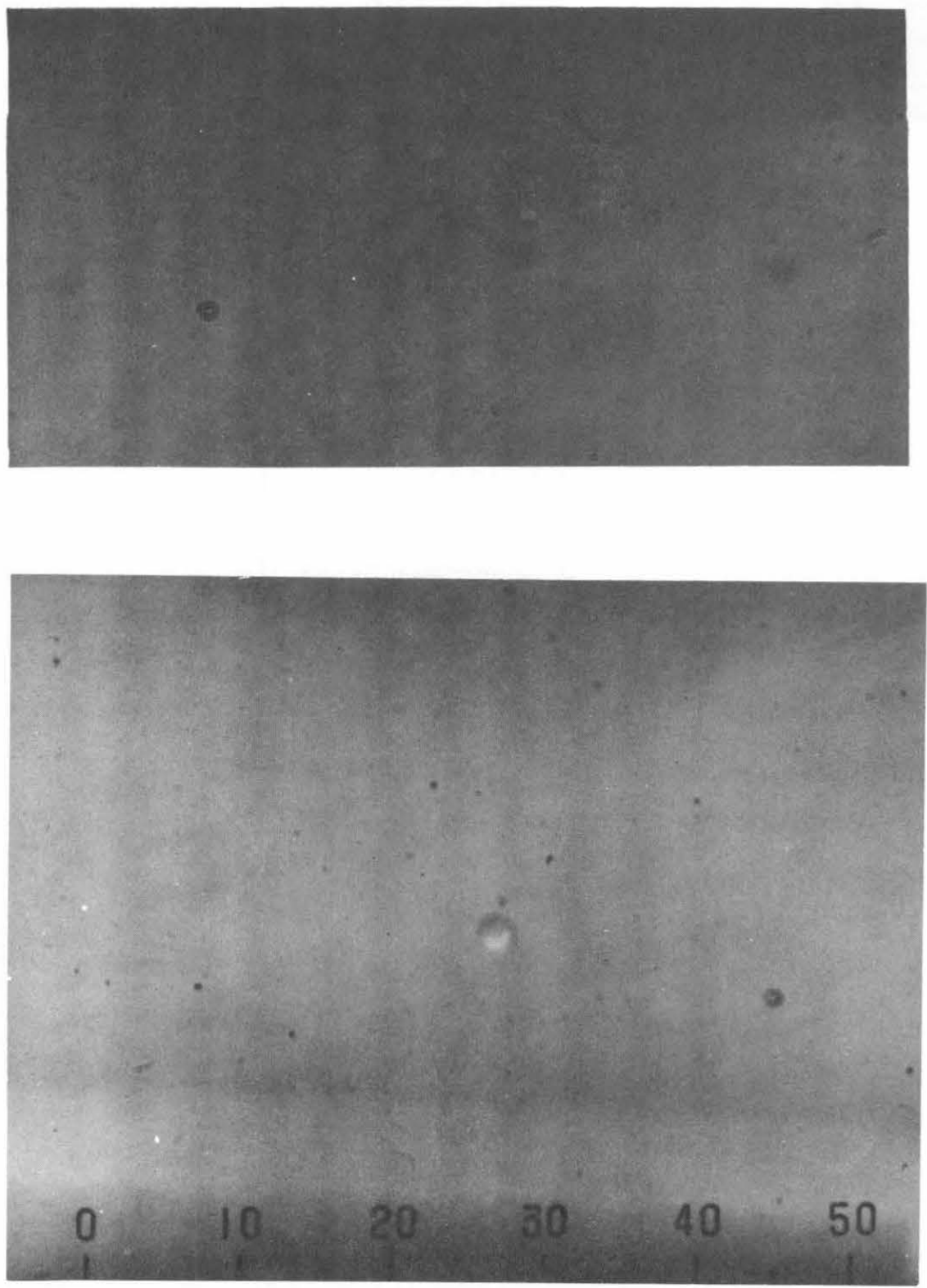

Figure 37: Photograph of Residual Cavities Taken at 200X. (Each sma11 division corresponds to $0.01 \mathrm{~mm}$. ) 

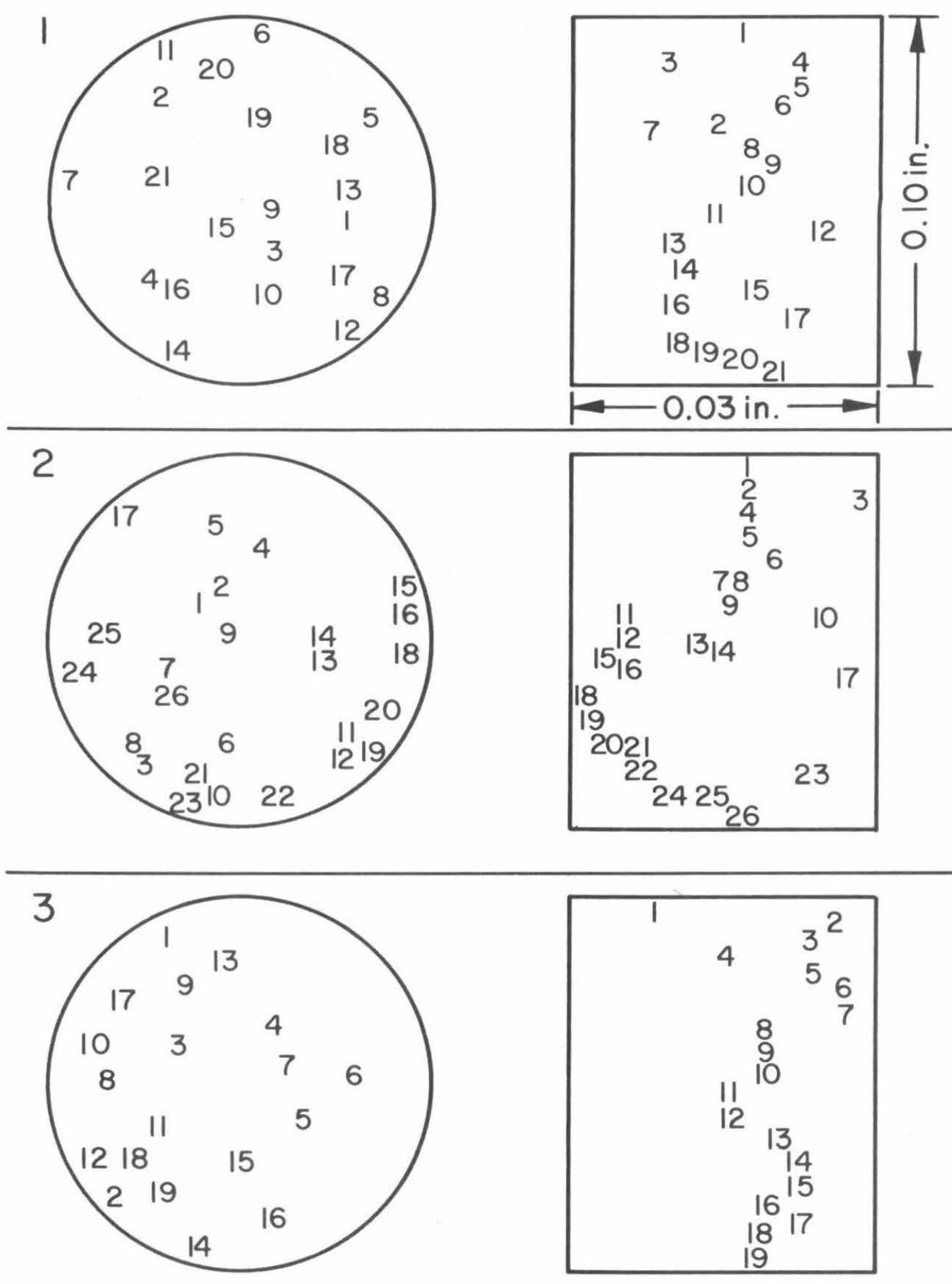

Figure 38. Typical Map of Distribution of Spherical Flaws Taken at Random Locations throughout the Specimens (150X). 
is approximately $7.6 \times 10^{-5}$. This is admittedly quite a small volume fraction, but at the same time there is supplied a large number of possible fracture nucleation points; viz. $30 \times 10^{4}$ voids per cubic inch. Although this number sounds large the cavities are usually 50 to 100 diameters or more apart. This makes it reasonable to assume that each flaw acts independently and that attention can be focused on a single cavity in making the fracture analysis. In other words, it will be hypothesized that fracture will initiate from one of these cavities and attention will only need to be given it. Furthermore the HT environment produced on the flaw in the poker chip will closely resemble that of Figure 39. The local field stress p is determined
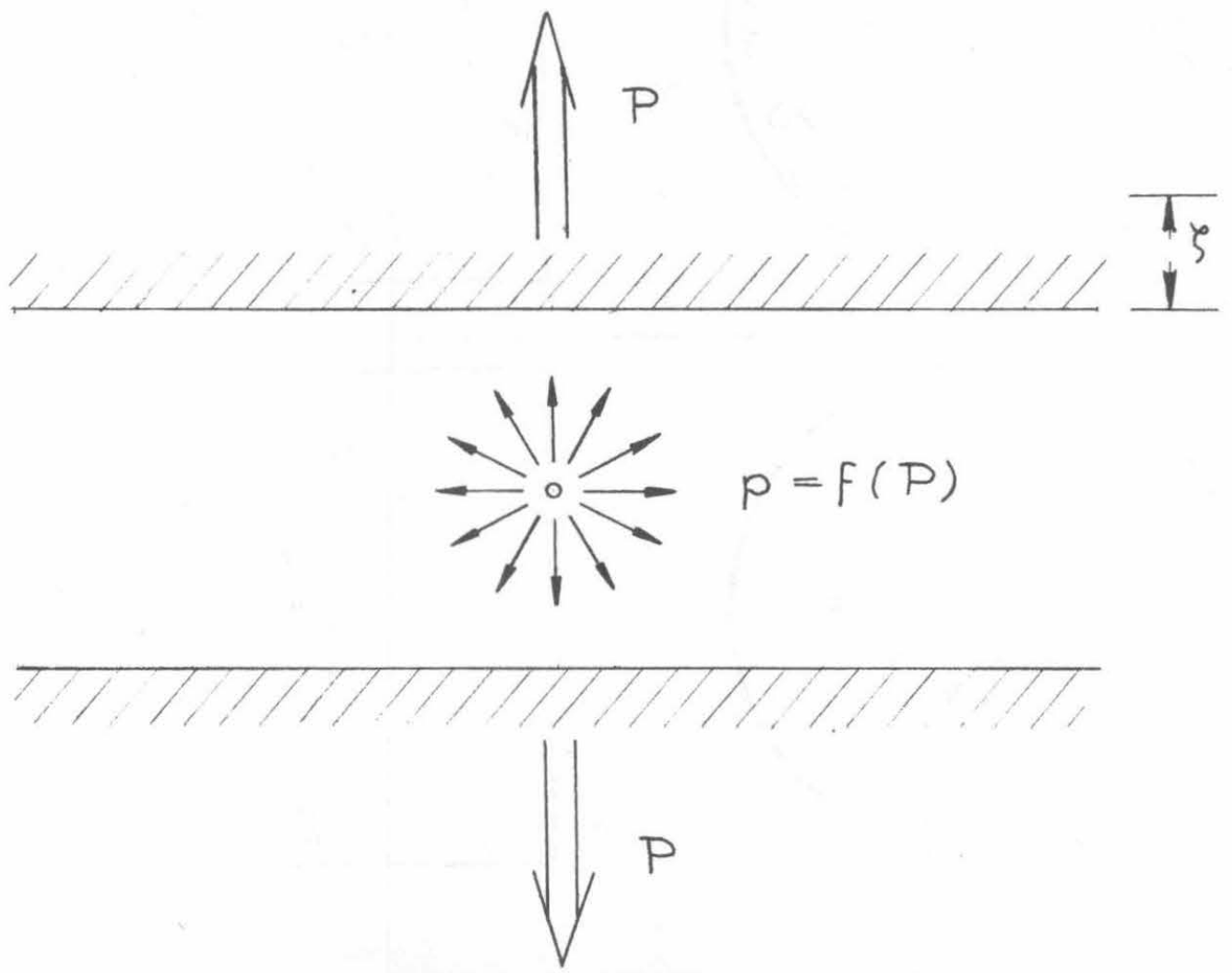

Figure 39. Schematic of Flaw in the Hydrostatic

Tensile Field in the Poker Chip. 
from the applied load $P$ or boundary displacement $\delta$ through Figure 8 . Then since the cavity is small compared to the specimen thickness, an analysis can be made considering the flaw to be alone in an infinite hydrostatic field, and the test will closely duplicate the theoretical assumptions and boundary conditions.

The approach to be followed will be to consider the transfer of energy for the cavity region during the fracture process. From this a critical value of the hydrostatic pressure will be predicted in terms of pertinent material and geometric parameters, indicating the point of rupture of the spherical flaw. However this will require some extensions of the classical concepts in order to deal with this particular geometry, stress field, etc., and a general discussion of the energetics of fracture is in order.

\section{ENERGY CRITERIA FOR ELASTIC FRACTURE}

To a novice in the field it appears that there is great need for elucidation of the basic principles upon which fracture energetics are built; so that extensions to other geometries, other stress fields, and other materials will be clear. Since this will have to be done in the instance of the spherical cavity, let us review a few fundamentals.

\section{Conservation of Energy}

Beginning with the First Law of Thermodynamics as applied to a general fracture process (65) it is possible to arrive at Griffith's classical results, (66) which were originally obtained in quite a different manner. The recoverable strain energy $U_{1}$, representing 
the strain energy before fracture, plus the work done by the surface tractions during fracture, $\delta W$, must, from the Conservation Law, equal the strain energy after fracture $\mathrm{U}_{2}$, plus the energy dissipated in forming new surface $\delta S$.

$$
\mathrm{U}_{1}+\delta \mathrm{W}=\mathrm{U}_{2}+\delta \mathrm{S}
$$

or forming an incremental equation

$$
\Delta U-\delta W+\delta S=0
$$

\section{Differential Form}

It is convenient for computation and for later comparisons to cast the energy equation into a differential form. This will be accomplished by dividing the equation by an increment of crack length and taking the limit as the change in crack length approaches zero. Implicit within this operation is the assumption that each term in the equation can be written as a continuous function of crack length, which can only be done if the flaw hypothesis is made. This results from the fact that the fracture point of a specimen with a residual crack is actually the point of initiation of the propagation phase, and the energy terms can be represented as continuous functions of crack length. On the other hand, if there is no microscopic flaw and fracture originates at a sub-continuum level, then the energy terms are not continuous functions of crack length, and the incremental equations must be used. The differential form of the conservation law for a fixed force boundary condition becomes, 
$\frac{d U}{d A}-F \frac{d \delta}{d A}+\gamma \frac{d A}{d A}=0$

where $F=$ Applied force at the boundary

$\delta=$ Displacement of the boundary

$\gamma=$ Surface energy density

$A=$ New surface created during fractures

\section{ENER GY FUNCTIONALS}

Consider now certain energy functionals which have been used in fracture analysis. The Potential Energy Functional can be considered as a starting point for Griffith's (66) discussion of fracture. In attempting to translate his discussion into mathematical terms we obtain,

$$
\bar{V}=U-P+S
$$

where $U$ = Strain energy

$$
\begin{aligned}
& \mathrm{P}=\text { Potential of the boundary load computed } \\
& \text { while the load is held constant } \\
& \mathrm{S}=\text { Surface energy } \\
& \delta \overline{\mathrm{V}}=\delta \mathrm{U}-\delta \mathrm{P}+\delta \mathrm{S}
\end{aligned}
$$

By a careful comparison of expression (4.2) and (4.5) and the physical processes which they describe, (30) it can be shown that $\delta \mathrm{P}=$ $\delta \mathrm{W}$. This is the crux of an argument that can be used to establish what Griffith assumed for the fracture criterion, i.e.,

$$
\delta \bar{V}\left[\frac{\partial U}{\partial c}-\frac{\partial P}{\partial c}+\gamma \frac{d A}{d c}\right] d c=0
$$


By a comparison with the energy equation (4.3), it is apparent that they are identical. This is a significant result, for it establishes that fracture predictions for fixed grip loading ${ }^{*}$ made on this basis of the adjusted potential energy being a minimum, are the same as obtained from energy conservation. At this point it is difficult to ascertain just why a minimization of $\bar{\gamma}$ should predict fracture, but a later examination of the stability aspects of the problem will clarify this point.

Extended Complementary Energy. If a similar comparison is made between the extended potential energy of equation (4.4) for fixed grip loading and the energy balance made for the same conditions, it can be demonstrated that the two do not coincide and that the two conditions are not the same. It is therefore concluded that the extended potential energy is not the correct functional for fixed grip loading. However an analogy can be made between the types of loading and the correct functional, for in the case of fixed force loading, where the force is held constant and the displacements are varied, it was found that the potential energy was the proper functional; therefore it would appear appropriate that for the fixed grip condition where the displacements are held constant and the forces are varying that complementary energy (49) should be considered.

It turns out that this is true. A direct comparison can be

* Griffith did not specify the boundary conditions with which he worked, but it can be deduced that it had to be fixed force. 
made and a minimization of an altered form of the complementary energy functional.

$$
\overline{\mathrm{V}}^{*}=\mathrm{U}-\mathrm{Q}+\mathrm{S}
$$

where $Q=\int_{\Sigma \delta} F_{i} \delta_{i} d \sigma$

$$
\begin{aligned}
\Sigma_{\delta}= & \begin{array}{l}
\text { Portion of the surface where displacements } \\
\text { are specified }
\end{array} \\
d \sigma= & \text { Differential surface element }
\end{aligned}
$$

coincides with the energy equation (4.3). At this point the physical reason why $\delta \overline{\mathrm{V}}^{*}=0$ predicts fracture is obscure, but it will be explained by considering the stability of the crack.

\section{STABILITY}

By means of the conservation law of energy, it has been possible to establish a necessary condition for fracture, since it is necessary that energy be conserved for the phenomenon to occur. Now it is of interest to inquire into the stability of the crack; whether it be in stable, neutral, or unstable equilibrium. Classically it is the second variation that provides information on stability, but this is not necessarily always the case. Sometimes the very nature of the problem will cause the first or possibly the first and second variations to vanish identically. (67) In fact, there is no limit to the number of variations that can vanish and consequently it is not a set principle as to which variation controls stability. This turns out to be the case in the general fracture problem that we are currently investigating. In order to see this, let us take a closer look 
at the physics, which reveals that the only ''resistance'' to be overcome is the surface energy, and until the other 'driving'' energy terms build up to it, a crack is perfectly stable. At the point of equality anything can happen and the crack at this critical stress is in neutral equilibrium. Anything above the equality point results in an unstable state for the crack, and it starts to propagate. Consequently in this case it is the first variation of the extended potential energy being set equal to zero that gives the stability criterion for the point of neutral equilibrium.

Buckling Analogy

The problem of elastic buckling theory for columns is a good analogy to fracture initiation and is helpful in clarifying this point of which variation is related to stability. By thinking of the potential energy of the column as a continuous function of the variation parameter, a qualitative plot can be made as shown in Figure 40 , and the potential energy can be expanded in a Taylor's series with the variation parameter acting as the independent variable. (67) This method gives a better physical insight into the problem and will make it easier to understand the analogous fracture problem.

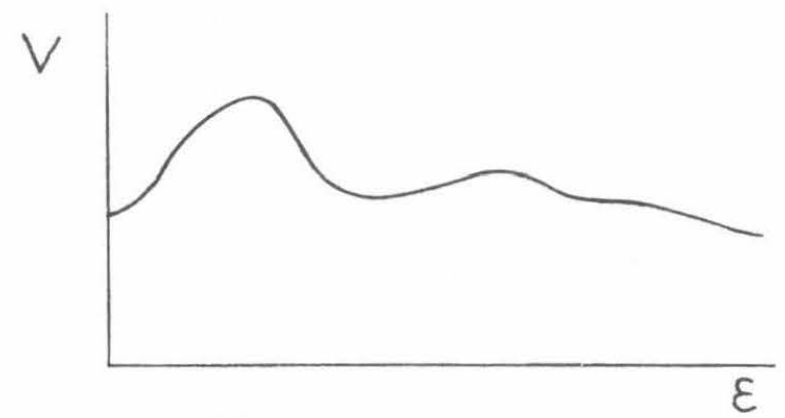

Figure 40. Arbitrary Plot of Potential Energy vs. the Variation Parameter. 
A Taylor's series expansion is readily written as

$$
\mathrm{V}=\mathrm{V}(0)+\mathrm{V}^{\prime}(0) \varepsilon+\mathrm{V}^{\prime \prime}(0) \frac{\varepsilon^{2}}{2 !}+\ldots
$$

However, by definition of the variation operator (68)

$$
\Delta \mathrm{V}=\delta \mathrm{V}+\delta^{2} \mathrm{~V}+\delta^{3} \mathrm{~V}+\ldots
$$

Thus if we are at an equilibrium point, the first variation vanishes and the sign of $\Delta \mathrm{V}$ is determined by the second variation, or in other words, the second variation gives information about stability. The one additional facet that affects the column buckling is that $\Delta \mathrm{V}$ is computed around a loading position when buckling is imminent; therefore $\Delta \mathrm{V}$ becomes a second order incremental change on top of the $\Delta V$ generated by loading from the natural state to the point of buckling. Consequently, if the potential energy is used, the first variation about the loaded state corresponds to a second variation about the initial state, so that the first variation for column buckling actually becomes equivalent to a second variation and gives the point of instability.

A similar set of circumstances prevails in the instance of imminent fracture, where we are using the extended potential energy. For, as in the case of buckling, the first variation about the point where fracture is imminent corresponds to a second variation about the natural state, and it is therefore the first variation that gives the information about crack stability. This can also be seen from the fact that the critical point using the extended potential energy was actually based upon the first law of thermodynamics, 
which implies that a state of equilibrium prevails. Interpreted in the light of a series expansion for $\bar{V}$, this would mean that the first variation, which is concerned with equilibrium, is automatically satisfied. Thus in the expansion the first term that appears is actually the second variation and is the one that is of interest when seeking information about stability. From this it is seen that for fracture prediction we are not dealing with equilibrium principles as Griffith discussed but stability, and now it is clear why the vanishing of the first derivative of the adjusted potential energy is the appropriate fracture condition.

In summary it may be stated that the conservation of energy provides a necessary condition for fracture as well as a basis upon which the potential energy can be extended; furthermore, the value at which the first variation of the new functional vanishes is the point of neutral equilibrium, demonstrating that the crack will run at this point, and this constitutes a type of sufficiency condition for fracture. Therefore the critical values predicted by this analysis are necessary and sufficient conditions for fracture within the limit of the degree to which the physics are modeled by the mathematics.

\section{ENERGY FRACTURE ANALYSIS - INFINITESIMAL THEORY}

We return now to consider the growth and eventual rupture of a spherical cavity in an infinite medium subjected to $\mathrm{HT}$ at infinity. Based upon the findings described previously in relation to the existence, location and distribution of spherical flaws, a boundary value problem formulated for this geometry would be expected to 
yield a solution that will accurately describe the physics. A mathematical model is depicted in Figure 41. The stress analysis (49) where the outer boundary is taken as spherical and of radius $\underline{b}$ yields

$$
\sigma_{r}=\frac{\mathrm{pb}^{3}\left(\mathrm{r}^{3}-\mathrm{a}^{3}\right)}{\mathrm{r}^{3}\left(\mathrm{~b}^{3}-\mathrm{a}^{3}\right)} \quad \sigma_{\mathrm{t}}=\frac{\mathrm{pb}^{3}\left(2 \mathrm{r}^{3}+\mathrm{a}^{3}\right)}{2 \mathrm{r}^{3}\left(\mathrm{~b}^{3}-\mathrm{a}^{3}\right)}
$$

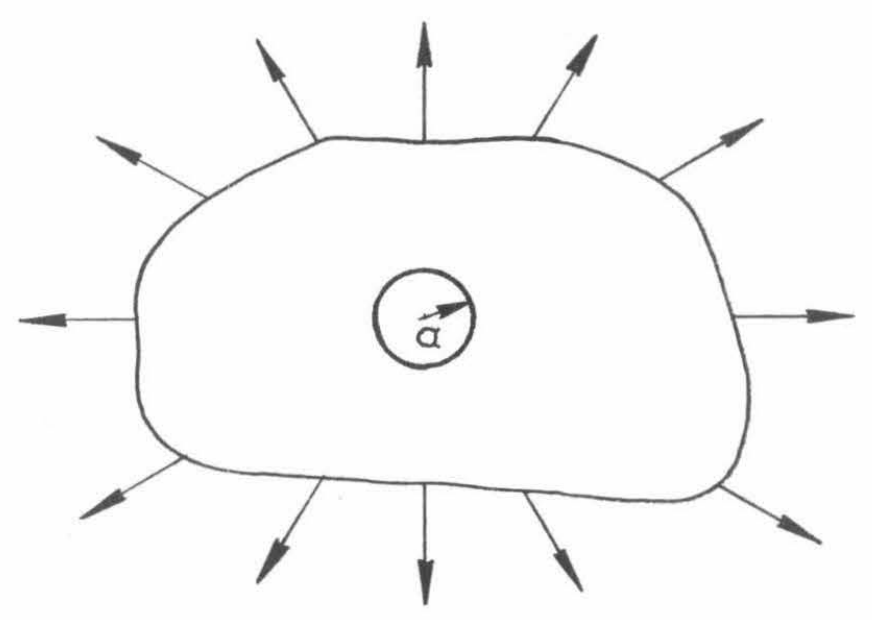

Figure 4l. Idealized Model of Spherical Flaw in a Hydrostatic Field.

The corresponding strains for an incompressible material become,

$$
\varepsilon_{r}=\frac{1}{E}\left(\sigma_{r}-\sigma_{t}\right) \quad \varepsilon_{t}=\frac{1}{2 E}\left(\sigma_{t}-\sigma_{r}\right)
$$

Forming a strain energy density function and integrating over the body we have

$$
U=\frac{1}{2} \int_{V} \tau_{i j} i j d V=\frac{\pi}{2 E} p^{2}\left[\frac{1}{a^{3}}-\frac{1}{b^{3}}\right]^{-1}
$$

\section{Critical Pressure}

In order to obtain the critical rupture pressure, the energy criterion of equation (4.6) is invoked 


$$
\frac{\partial}{\partial \mathrm{a}}(\mathrm{U}-\mathrm{S})=0
$$

where for a linearly elastic material $P=-2 U$. (69) $S$ represents the energy required to create unit surface, and a is a measure of the flaw growth. In this instance when $\mathrm{b} \gg \mathrm{a}$, equation (4.12) becomes

$$
\frac{\partial}{\partial a}\left[\frac{3 \pi}{2 \mathrm{E}} \mathrm{p}^{2} \mathrm{a}^{3}-4 \pi \mathrm{a}^{2} \mathrm{\gamma}\right]=0
$$

which yields a critical pressure of

$$
p_{c}=\frac{4}{3} \sqrt{\frac{E y}{a}}
$$

Flaw Size Dependency

This gives the typical inverse square root dependency of critical rupture stress on initial flaw radius. In fact, the defining equation of (4.13) varies only slightly for the entire spectrum of flaw geometries. Sneddon (70) and Sack (71) obtained the inverse square root dependency for ''penny-shaped' 'cracks in hydrostatic fields, which is very similar to the Griffith $(66,72)$ result for the line crack in a two-dimensional sheet.

A summary table shows the quantitative similarity for representative geometries (73)

$\begin{array}{ccccc}\text { Geometry } & \begin{array}{c}\text { Sheet with } \\ \text { line crack } \\ \text { (Griffith) }\end{array} & \begin{array}{c}\text { Cylindrical } \\ \text { cavity }\end{array} & \begin{array}{c}\text { Penny-shaped } \\ \text { crack } \\ \text { (Sneddon) }\end{array} & \begin{array}{c}\text { Spherical } \\ \text { cavity }\end{array}\end{array}$
Critical
Stress
$\sqrt{\frac{2}{\pi} \frac{E \gamma}{a}}$
$\sqrt{\frac{E \gamma}{2 a}}$
$\sqrt{\frac{2 \pi \mathrm{EY}}{3 a}}$
$\frac{4}{3} \sqrt{\frac{E \gamma}{a}}$

Williams (73) has discussed this similarity and uses it to investigate the more complicated phenomenon of viscoelastic fracture. 
Mode of Propagation

It should be noted that the analysis inherently assumes a radial mode of propagation for the fracture surface; i.e., that the flaw grows spherically in a manner similar to ablation. This may place a limitation on the information gained, but a similar situation arises for the "'penny-shaped'' crack, which also is confined to propagate radially in a planar mode. Only experimentation can provide the means for evaluating these assumptions and determining what portion or portions of the fracture process can be analyzed by them.

ENER GY FRACTURE ANALYSIS - EFFECT OF FINITE DEFORMATION

The spherical symmetry in this problem makes it possible to extend the infinitesimal deformation fracture analysis to include effects of finite deformations. This is seldom possible, although Gent and Lindley (24) did use the model of a spherical cavity in an infinite medium and investigated the strains at the cavity using a maximum strain criterion. Schapery and Williams (74) used a nonlinear theory for the same problem and coupled it with an energy criterion to predict fracture. This is a particularly interesting approach for polymeric materials where large strains are the rule; furthermore, it provides an opportunity to see how large strains affect the inverse square root dependency of initial flaw size.

Finite Strain Effects

The presence of deformations exceeding the limits of infinitesimal theory produces many ramifications in the mechanics of the 
energy analysis: First, the matter of loading requires a more careful definition to fit the actual conditions both experimentally and theoretically, for with finite strains the change in area of the bounding surfaces is accounted for and there becomes a distinction between fixed force and fixed stress conditions. This difference will manifest itself in almost every quantity previously calculated and will affect the outcome of the conclusions drawn on the basis of the infinitesimal theory.

Second, this same matter of the differences between the deformed and the undeformed area produces questions about the manner in which surface energy is handled in the governing expressions. Normally in these computations, a surface energy is computed as if it were a variable of state; i.e., as if the existing surface possessed a given surface energy as opposed to only speaking of changes in the surface, or energy required to create surface. The actual computation is then made by differentiating this quantity with respect to the crack length. However additional consideration must be given when the surface of interest is significantly altered by deformation before fracture occurs, i. e., the original surface changes in area but does not rupture. This deformation is accounted for in the stress analysis of the body and in the energy expressions, but the correct manner in which it influences the surface energy may be debatable.

Third, the classical potential energy theorem has been shown to apply regardless of the magnitude of strain. For example, Green and Zerna (75) derived the theorem for large strains, and the form of the expression is found to be identical with the linear 
theory if proper attention is given to the definition of stress and strain. By examining Figure 42, which depicts a loading history and subsequent fracture under fixed force conditions for a general non-linear body, it is seen that $\delta P$ of the potential energy expression (4.6) is still equal to $\delta W$ of the conservation equation (4.2). As a result, the first variation of the extended potential energy,

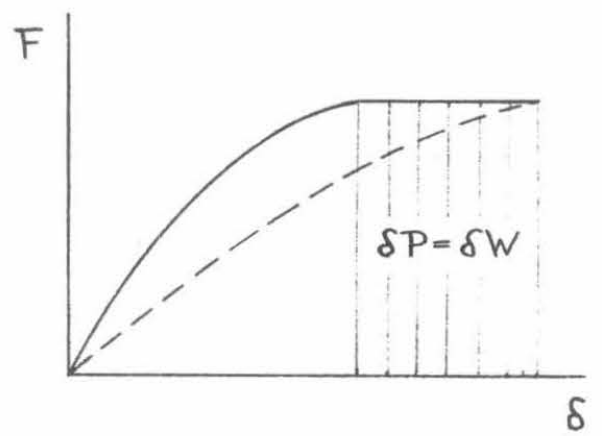

Figure 42. Force-Deflection Curve.

with proper stress and strain definition, still remains as a valid stability criterion for large strains also.

Fourth, the complementary energy functional for finite strains has just been formulated by Levinson (76) in terms of the Lagrange stress and strain tensor. The Lagrange stress tensor is an unsymmetric tensor associated with base vectors in the undeformed body, whose intensity is measured in terms of the undeformed area. The Lagrange strain tensor is simply the displacement gradient. Levinson was able to obtain a complementary energy principle, which differs in form from the infinitesimal functional only by a change in sign. With regard to this he states, 'This is because it is customary to give the infinitesimal theorem 
as a minimum principle, (for stable equilibrium) whereas its derivation by the Legendre transformation would lead to a maximum principle whose function would agree in sign."' Thus the first variation of $\bar{V}^{*}$ set equal to zero applies for finite strains also, since it checks energy conservation laws if proper care is given to stress and strain definition.

Fifth, the fracture stress for the different loading conditions is no longer the same when non-linearities are considered, whether they be finite or infinitesimal. This can be shown through a comparison of the energy equations for the limit cases of fixed force and fixed grip. (See Figure 43.) For purposes of illustration,

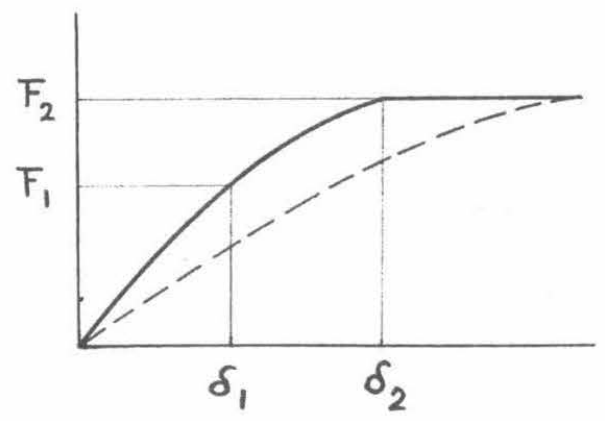

Figure 43. Comparison of Failure under FixedForce and Fixed Displacement Boundary Conditions assume the force deflection equation to be

$$
F=k(c) f(\delta)
$$

The strain energy is still equal to the work done on the body

$$
U=\int F d \delta=k(c) \int f(\delta) d \delta=k(c) g(\delta)
$$

or 


$$
U=F \frac{g(\delta)}{f(\delta)}=F h(\delta)
$$

The energy expressions from equation (4.2) become

$$
\begin{aligned}
& \left\{\mathrm{F} \frac{\mathrm{dh}}{\mathrm{d} \delta}\left(\frac{\mathrm{d} \delta}{\mathrm{dc}}\right)_{\mathrm{F}}-\mathrm{F}\left(\frac{\mathrm{d} \delta}{\mathrm{dc}}\right)_{\mathrm{F}}+\gamma\left(\frac{\mathrm{dA}}{\mathrm{dc}}\right)_{\mathrm{F}}\right\}_{\mathrm{F}=\mathrm{F}_{2}}=0 \text { (Fixed Force) } \\
& \left\{\mathrm{h}(\delta)\left(\frac{\mathrm{dF}}{\mathrm{dc}}\right)_{\delta}+\gamma\left(\frac{\mathrm{dA}}{\mathrm{dc}}\right)_{\delta}\right\}_{\delta=\delta_{1}}=0 \quad \text { (Fixed Grip) }
\end{aligned}
$$

Since

$$
\gamma\left(\frac{\mathrm{dA}}{\mathrm{dc}}\right)_{\mathrm{F}}=\gamma\left(\frac{\mathrm{dA}}{\mathrm{dc}}\right)_{\delta}
$$

Then

$$
\left\{\mathrm{F}\left(\frac{\mathrm{dh}}{\mathrm{d} \delta}-1\right)\left(\frac{\mathrm{d} \delta}{\mathrm{dc}}\right)_{\mathrm{F}}\right\}_{\mathrm{F}=\mathrm{F}_{2}}=\left\{\mathrm{h}(\delta)\left(\frac{\mathrm{dF}}{\mathrm{dc}}\right)_{\delta}\right\}_{\delta=\delta_{1}}
$$

But $\quad \frac{d h}{d \delta}=1-\frac{g(\delta)}{f^{2}(\delta)} \frac{d f}{d \delta}$

Substituting (4.15) and (4.19) into (4.18)

$$
\left\{-F \frac{g(\delta)}{f^{2}(\delta)} \frac{d f}{d \delta}\left(\frac{d \delta}{d c}\right)_{F}\right\}_{F=F_{2}}=\left\{h(\delta) f(\delta) \frac{d k}{d c}\right\}_{\delta=\delta_{1}}
$$

Returning to the equivalent constitutive law, equation (4.15), a relationship between $\frac{\mathrm{d} \delta}{\mathrm{dc}}$ and $\frac{\mathrm{dk}}{\mathrm{dc}}$ can be found

$$
\left\{\frac{d F}{d c}\right\}_{F}=0=k\left\{\frac{d f}{d c}\right\}_{F}+f \frac{d k}{d c}
$$

which, when substituted in (4.20) and simplified, gives 


$$
F_{2}=\frac{h\left(\delta_{1}\right)}{h\left(\delta_{2}\right)} F_{1}
$$

The conclusion which follows is that in general for non-linear systems, the fracture stress depends upon the form of the stressstrain law as well as the boundary loads, and the specific cases must be analyzed. For instance, if $f(\delta)=\delta^{1 / n}$ the critical stresses coincide, but the nature of the system must always be examined to make certain. This could possibly explain some discrepancies in polymeric fracture data, for in the instance of typical material representations such as Neo-Hookean or Mooney-Rivlin, the critical loads will not coincide because of the form of the constitutive law.

\section{CRITICAL FRACTURE POINT}

The critical fracture stress will now be computed for both a Neo-Hookean (NH) and a Mooney-Rivlin (MR) material to provide not only a comparison between the two non-linear stress-strain laws, but also with the infinitesimal theory. The strain energy function for both can be represented by

$$
W=\frac{E}{6}\left[+\left(I_{1}-3\right)+(1-f)\left(I_{2}-3\right)\right] \quad I_{3}=1
$$

where $f=1$ gives $\mathrm{NH}$, and $0 \leqslant f<1$ is MR. Following Levinson (78) the relation between the hydrostatic tensile pressure, p, applied at infinity and the stretch ratios of the inner boundary a and outer boundary $\underline{b}$ is

$$
\frac{p}{\mu}=\frac{f}{2}\left[\left(\frac{1}{\lambda_{b}^{4}}-\frac{1}{\lambda_{a}^{4}}\right)+4\left(\frac{1}{\lambda_{b}}-\frac{1}{\lambda_{a}}\right)\right]-(1-f)\left[\left(\frac{1}{\lambda_{a}^{2}}-\frac{1}{\lambda_{b}^{2}}\right)-2\left(\lambda_{a}-\lambda_{b}\right)\right]
$$


From incompressibility

$$
b_{0}^{3}-a_{0}^{3}=b^{3}-a^{3}
$$

where $a_{0}, b_{0}$ denote undeformed measures of the radii. This can be rewritten as

$$
a_{0}^{3}\left(\lambda_{a}^{3}-1\right)=b_{0}^{3}\left(\lambda_{b}^{3}-1\right)
$$

or

$$
\lambda_{b}=\left[1+\left(\frac{a_{0}}{b_{0}}\right)^{3}\left(\lambda_{a}^{3}-1\right)\right]^{1 / 3}
$$

However for an infinite medium $\mathrm{a}_{0} / \mathrm{b}_{0}<<1$; furthermore, $\mathrm{a}_{0} / \mathrm{b}_{0}=$ $\left(\frac{\lambda_{a} a_{0}}{b_{0}}\right) \ll 1$. From the binomial expansion

$$
\lambda_{\mathrm{b}}^{\mathrm{n}}=1+\frac{\mathrm{n}}{3}\left(\frac{\mathrm{a}_{0}}{\mathrm{~b}_{0}}\right)^{3}\left(\lambda_{\mathrm{a}}^{3}-1\right)+\ldots
$$

Substituting $(4.27)$ into $(4.23)$ the pressure relation simplifies to

$$
p=\frac{E}{6} f\left[5-\frac{4}{\lambda_{a}}-\frac{1}{\lambda_{a}^{4}}\right]-\frac{E}{3}(1-f)\left[\frac{1}{\lambda_{a}^{2}}-2 \lambda_{a}+1\right]
$$

Strain Energy. As was previously discussed, the altered or extended form of the potential energy functional can also be used for finite deformations. Of course the boundary conditions remain as constant pressure during fracture and the potential of the surface forces is no longer twice the strain energy as in the linear theory. All three energy terms must now be individually computed and inserted into the governing energy equation (4.6)

$$
\frac{\partial}{\partial a_{0}}[U-P+S]=0
$$


where $U=$ Strain Energy

$P=$ Potential of Surface Forces

$\mathrm{S}=$ Surface Energy

The strain energy is computed through the defining integral

$$
\mathrm{U}=\int_{\mathrm{b}_{0}}^{\mathrm{b}} 4 \pi \mathrm{pb}^{2} \mathrm{db}=\int_{1}^{\lambda_{0}} 4 \pi \mathrm{pb}_{0}^{3} \lambda_{\mathrm{b}}^{2} \mathrm{~d} \lambda_{\mathrm{b}}
$$

Using (4.27) to express the integral in terms of the extension ratio at the flaw cavity, $(4.30)$ becomes

$$
\mathrm{U}=4 \pi \mathrm{b}_{0}^{3} \int_{1}^{\lambda_{\mathrm{a}}} \mathrm{p} \lambda_{\mathrm{a}}^{2}\left(\frac{\mathrm{a}_{0}}{\mathrm{~b}_{0}}\right)^{3} \mathrm{~d} \lambda_{\mathrm{a}}
$$

where the fact that $a_{0} / b_{0} \ll 1$ has been used to simplify the expression. Substituting the expression for p, equation (4.28), and integrating

$$
U=\frac{4}{3} \pi a_{0}^{3} E\left[(1-f) \frac{\lambda^{4}}{2}+\frac{(7+-2)}{6} \lambda_{a}^{3}-f \lambda_{a}^{2}-(10 f) \lambda_{a}+\frac{f}{2 \lambda_{a}}-\frac{(7 f-5)}{6}\right]
$$

\section{Potential of Surface Forces. This is a much simpler term} to compute due to the fact that $\mathrm{p}$ is now held constant.

$$
\mathrm{P}=4 \pi \mathrm{pb}_{0}^{2}\left(\mathrm{~b}-\mathrm{b}_{0}\right)=4 \pi \mathrm{pb}_{0}^{3}\left(\lambda_{\mathrm{b}}-1\right)
$$

Using (4.27) once again

$$
\mathrm{P}=\frac{4}{3} \pi \mathrm{pa}_{0}^{3}\left(\lambda_{\mathrm{a}}^{3}-1\right)
$$

Surface Energy. One of the new facets of the problem alluded to previously is encountered at this point. Before rupturing, the 
cavity surface may undergo a large change in surface area, which would be considered as a stretching of the molecular structure, with all input energy remaining recoverable. At the critical point, energy will be dissipated by the formation of new surface, and although the cavity is deformed considerably, the surface energy density should be referred to the cavity surface before deformation. The reason being that $\gamma$, the surface energy density, is an artifice that relates molecular activity to continuum activity. Consequently it is the number of molecules present on the surface that is of concern in computing fracture energies. This remains constant during the deformation and therefore

$$
\mathrm{S}=4 \pi \mathrm{a}_{0}^{2} \gamma
$$

\section{CRITICALITY EQUATION}

Substituting equations (4.32), (4.34) and (4.35) into the energy equation of (4.29) and performing the differentiation, with $\mathrm{p}$ held constant, we obtain

$$
\lambda_{a}^{8}+\frac{2 f}{1-f} \lambda_{a}^{6}-\frac{3+2 k}{1-f} \lambda_{a}^{4}+2 \lambda_{a}^{2}+\frac{f}{1-f}=0
$$

This expresses the critical condition for fracture in terms of the stretch ratio at the cavity and a material parameter $\mathrm{k}=\frac{6 \mathrm{y}}{\mathrm{Ea}} \cdot$ The simplest case is for the $\mathrm{NH}$ material when $f=1$, then the critical condition is (41)

$$
\lambda_{a}^{6}-\left(\frac{3}{2}+\mathrm{k}\right) \lambda_{\mathrm{a}}^{4}+\frac{1}{2}=0
$$

A check of this polynomial reveals that there is only one 
positive, real root greater than one, so no complicated interpretations arise. Of course once the critical extension ratio is known, the critical pressure can be computed from equation (4.28), and for completeness, the expression for the tangential hoop stress is given.

$$
\sigma_{t}=\mu\left[f+\lambda_{a}^{2}(1-f)\right]\left[\lambda_{a}^{2}-\frac{1}{\lambda_{a}^{4}}\right]
$$

Comparative Results. All of these quantities have been plotted collectively for comparison in Figures $44-46$. There is a noticeable variation in almost every quantity, as for instance the ultimate extension of the cavity surface for $\mathrm{NH}$ bodies is increased considerably over MR bodies for $\log \frac{6 \mathrm{~T}}{\mathrm{Ea}_{0}}$ greater than 0.50 , while the reverse relation holds for the hydrostatic field stress p. In this case, p for the $\mathrm{NH}$ body approaches an asymptote of $\frac{5 \mathrm{E}}{6 \mathrm{E}}$, while MR exhibits behavior characteristic of the infinitesimal theory and as a result these two curves diverge rapidly. However for both $\mathrm{NH}$ and $\mathrm{MR}$ the inverse square root dependency on $\mathrm{a}_{0}$ is reduced by considering finite strains. In spite of this reduction, there still remains a strong influence of $\mathrm{a}_{0}$ on $\mathrm{p}_{\mathrm{c}}$.

The validity of these predicted fracture levels can only be ascertained by experiment. In this connection, a method was conceived for inserting artificial flaws of varying sizes into the poker-chip specimen and subjecting them to HT. Details of these results, to be described subsequently, shed light on the fracture behavior of spherical cavities and provide further information for evaluating the flaw analysis. 


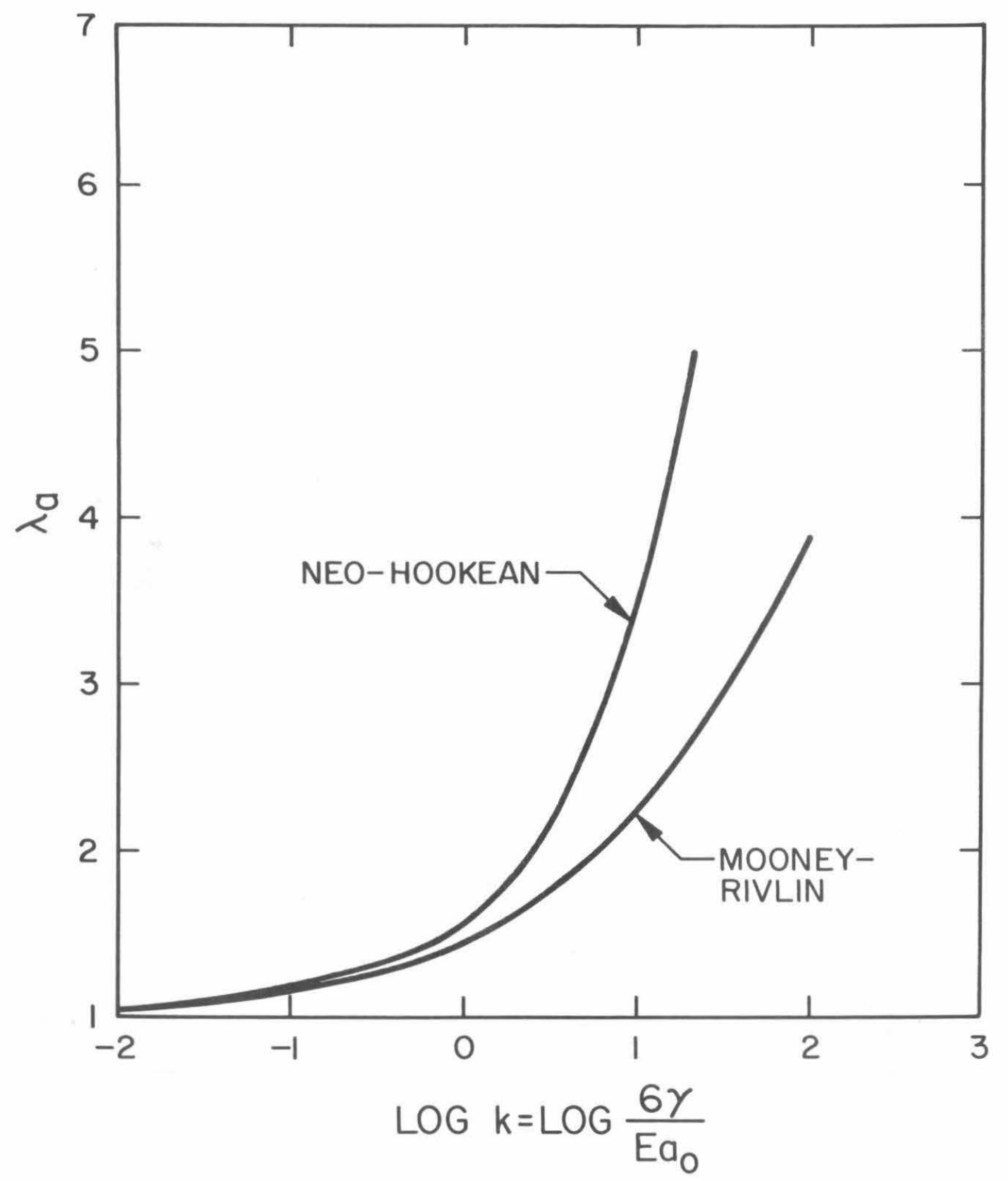

Figure 44. Critical Extension Ratio at the Surface for Instability of a Spherical Cavity. 


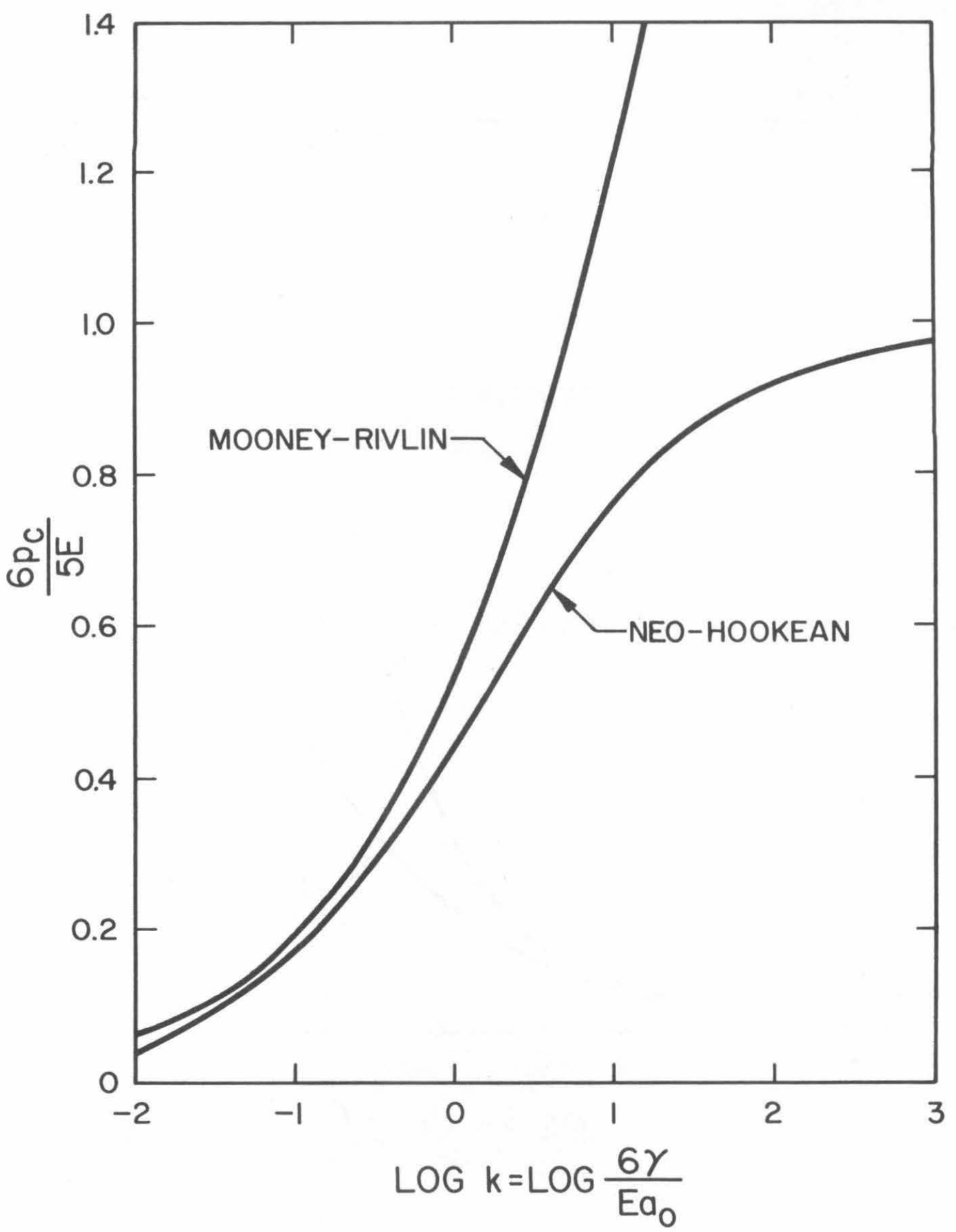

Figure 45. Critical Hydrostatic Pressure Condition for Instability of a Spherical Cavity. 


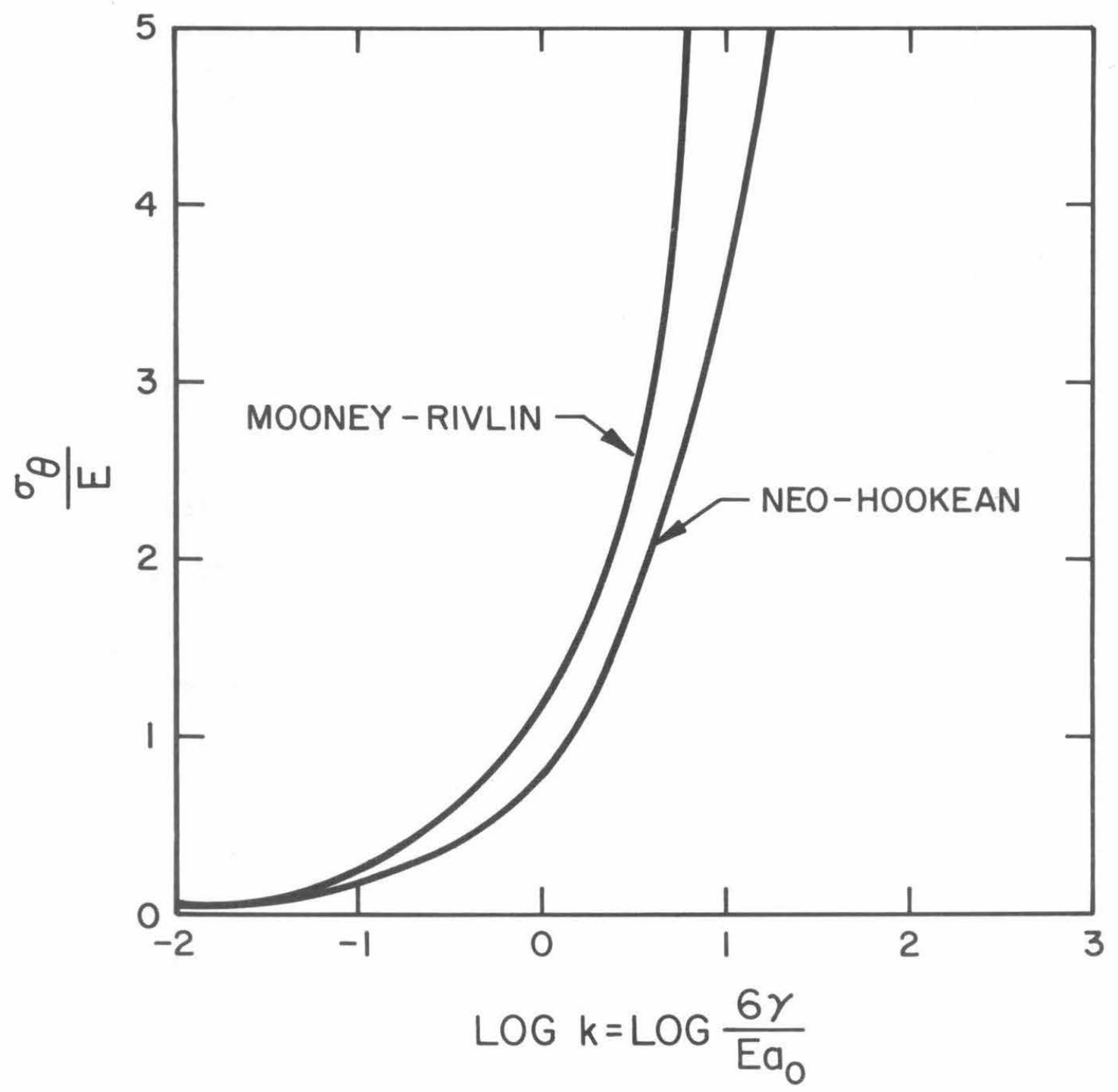

Figure 46. Critical Hoop Stress for Instability of a Spherical Cavity. 
CHAPTER V

ARTIFICIAL FLAWS

As a means of further assessing the theoretical treatment, as well as serving as a vehicle for the study of flaw behavior, a number of poker-chip specimens were prepared with a dominant central flaw in the form of an air bubble. In order for experiments for such a specimen to be meaningful, the cavities must be inserted without disturbing the normal manufacturing operations of the standard material. It will be recalled that the standard manufacturing operation consisted of mixing together the two components, pre-polymer and catalyst, under an inert atmosphere of nitrogen. The mix is then transferred to a preheated mold through piping that enables the mold to be filled while in the oven. The specimen is then cured at $150^{\circ} \mathrm{C}$ for two hours. The bubbles were inserted into the material without interrupting this operation by constructing a mold with a glass front as shown in Figure 47.

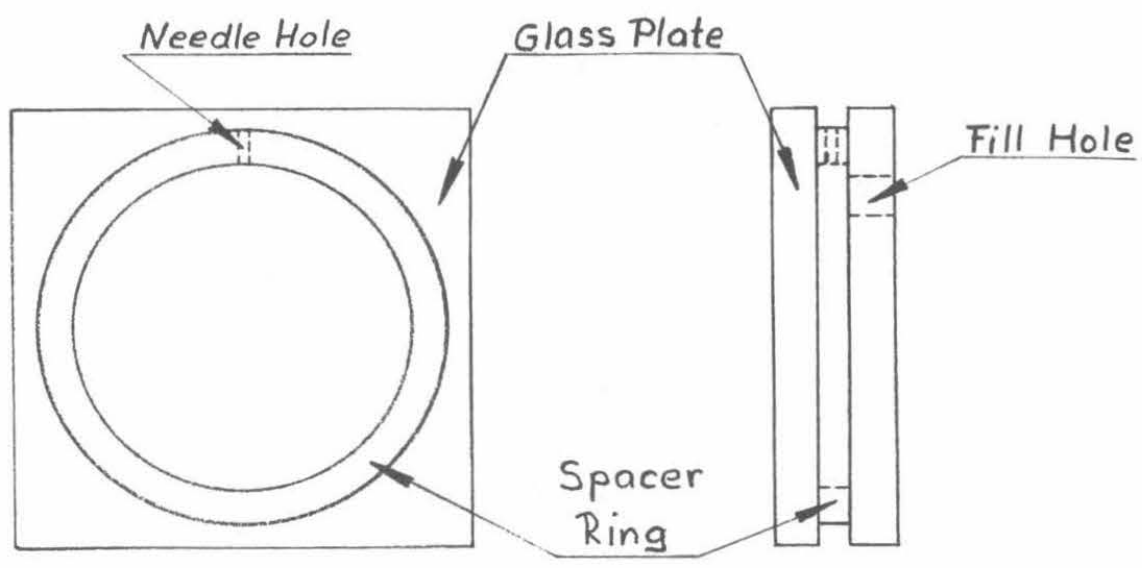

Figure 47. Mold Assembly 
The spacer ring was fitted with a hole to admit a long hypodermic needle, which was of sufficient length to reach from a syringe outside of the oven to the inner parts of the mold. In the apparatus used, the needle was two feet long. The two components were mixed and preheated as before, and the preheated glass front mold was filled while in the oven in the usual manner. The polymerization and cross-linking process begin to take place immediately upon mixing of the two components; however, there is a pot life during which the material will flow even though it is becoming more viscous with time. It is while the polymer is still in this liquid state that the hypodermic needle is inserted into the mold and a bubble blown from outside the oven. The needle is withdrawn before the liquid has become solid enough to retain any memory of its presence. The mold was mounted on a shaft capable of rotation by an electric motor controlled from the outside of the oven. In this way, the location of the bubble in the mold could be controlled, as it had a tendency to rise during the early stages before the polymer became solid. The complete arrangement is shown in Figure 48.

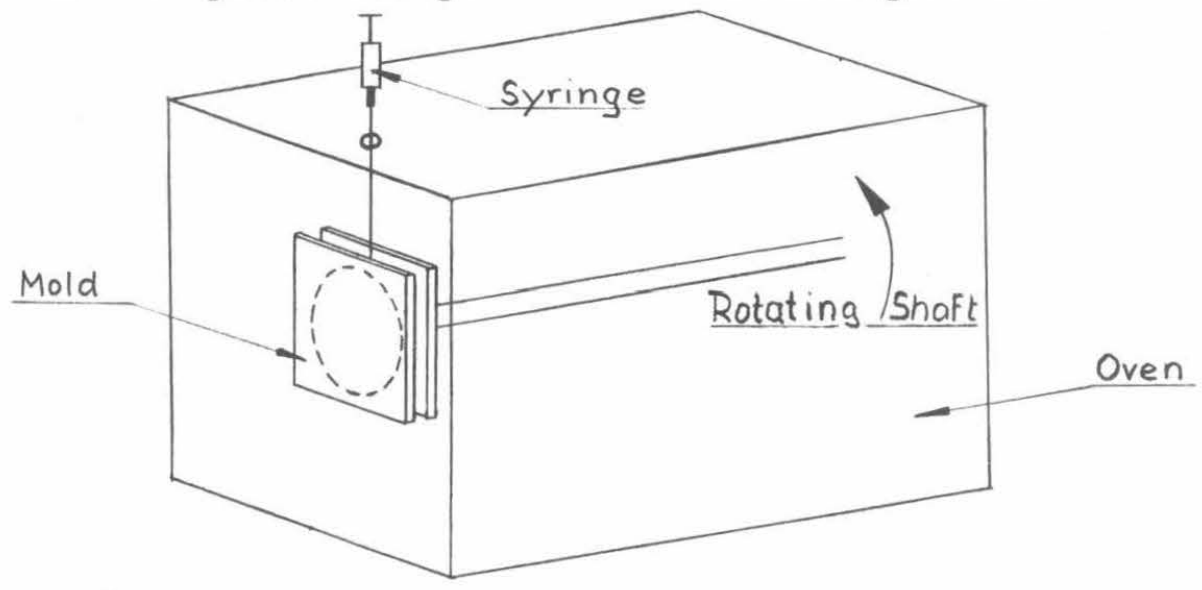

Figure 48. Schematic of Apparatus used for Inserting Artificial Flaws 
Following this operation, the specimen was cured in the standard manner at $150^{\circ} \mathrm{C}$ for two hours, and then removed from the mold and stored in a dry box until used.

\section{OBSERVATIONS}

The specimen was mounted between lucite grips as before with the dominant flaw located in the center. Direct visual observation of the growth of the flaw under load was made by means of a portable microscope from which several interesting things were learned: 1) Deformations at the surface of the cavity were definitely finite, with strains ranging up to 150 percent. This is quite unusual since the maximum strain in uniaxial tension attains values of only 35 to 40 percent, but it appears that the necessary energy condition for fracture is not met in this configuration until a significantly larger strain. It should be emphasized, in light of the stress axis theorem discussed previously, that these are the local strains around the cavity and not the global strains measured at the boundaries, which were still small in this test. 2) Fracture levels were not affected by the presence of the dominant flaw but were unchanged from those of the regular specimens. 3) Fracture did not occur at the artificial flaw but elsewhere in the specimen, sometimes close and sometimes distant from the bubble. Even in the few cases where multiple artificial flaws were inserted in the same specimen, fracture still occurred in the field rather than on the surface of one of these cavities. 4) None of the cracks after nucleating elsewhere ever propagated into or through a bubble; all 
bubbles remained intact and could be seen unruptured after the specimen was completely severed into two pieces. This led to the examination of fracture of the material in other geometries to determine if the cracks had propagated through any of the residual bubbles regardless of the initial size, but no evidence could be found. A behavior of this nature was noted by Schwarzl and Staverman (79) in Plexiglas but in this instance it was concluded that the bubbles are not a mechanism of propagation; in fact, almost the opposite is true. The propagating surface seems to either stop or go around such a discontinuity in the material.

It should be noted in the interpretation of these results that the dominant flaw was still surrounded by a matrix of the residual flaws, since the material was produced in a standard way. The large cavity was not alone in the field, and it is felt that a significant difference in behavior would have been noted had it been alone. Since there still remained the distribution of tiny bubbles, invisible to the naked eye, the experiments are taken as evidence of the validity of a so-called Saturn-ring theory of crack propagation.

\section{SATURN-RING CRACK}

Assuming as before that the flaws are dispersed widely enough not to interfere with each other or cause disturbances in the hydrostatic stress field, we will focus our attention on a single cavity in an infinite field that will eventually trigger the fracture. Let us visualize a reasonable physical mechanism by which fracture can occur by picturing the cavity as it grows under the action of the hydrostatic tension. As the pressure is increased, the cavity 
will grow symmetrically until virtually all points on the surface are brought to imminent rupture. It has been well documented in almost every experimental test made on rubber that the plane of fracture occurs perpendicular to the plane of the maximum principal stresses which, for a cavity in a symmetric field, is the tangential hoop stress at the surface. In order for the fracture to take place perpendicular to the maximum tensile stress, the fracture plane must be oriented so as to pass through the center of the cavity, and thus it will be located on a great circle of the sphere, presumably starting at some locally weak point on this great circle. It can then be imagined that the crack will travel around the sphere in the form of a Saturn-ring, (see Figure 49), quickly opening the sphere and propagating in a planar, radial direction away from the

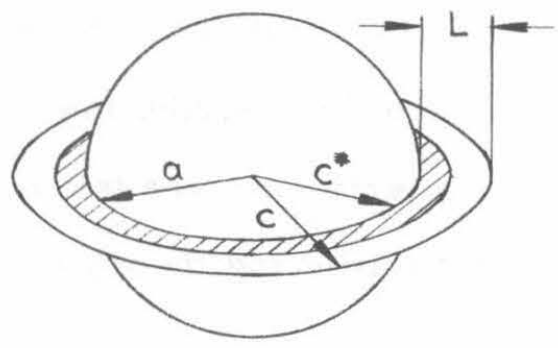

Figure 49. Saturn-Ring Crack

spherical surface. We will term this 'the Saturn-ring mode of propagation." This obviously is an approximation to the real process for it would never occur in such a symmetric fashion but would be much more complicated; however, it appears to be a reasonable model upon which to build a mathematical formulation and from which the salient features of the problem can be studied. 
Strain Energy Function

The boundary value problem of a spherical hole with a Saturnring, planar crack has not been solved even for infinitesimal deformations. It will therefore be necessary to slightly extend, in an ad hoc manner, the results of other similar elasticity problems that have been solved in order to obtain an estimate of the stress field for the geometry of Figure 46. This will be done by examining the interrelationship among three known solutions: (i) a body in plane strain possessing an internal crack similar to that used by Griffith, (ii) a body in plane strain with a cylindrical hole and two cracks extending radially from the hole as shown in Figure 50, and (iii) the three-dimensional penny-shaped crack.

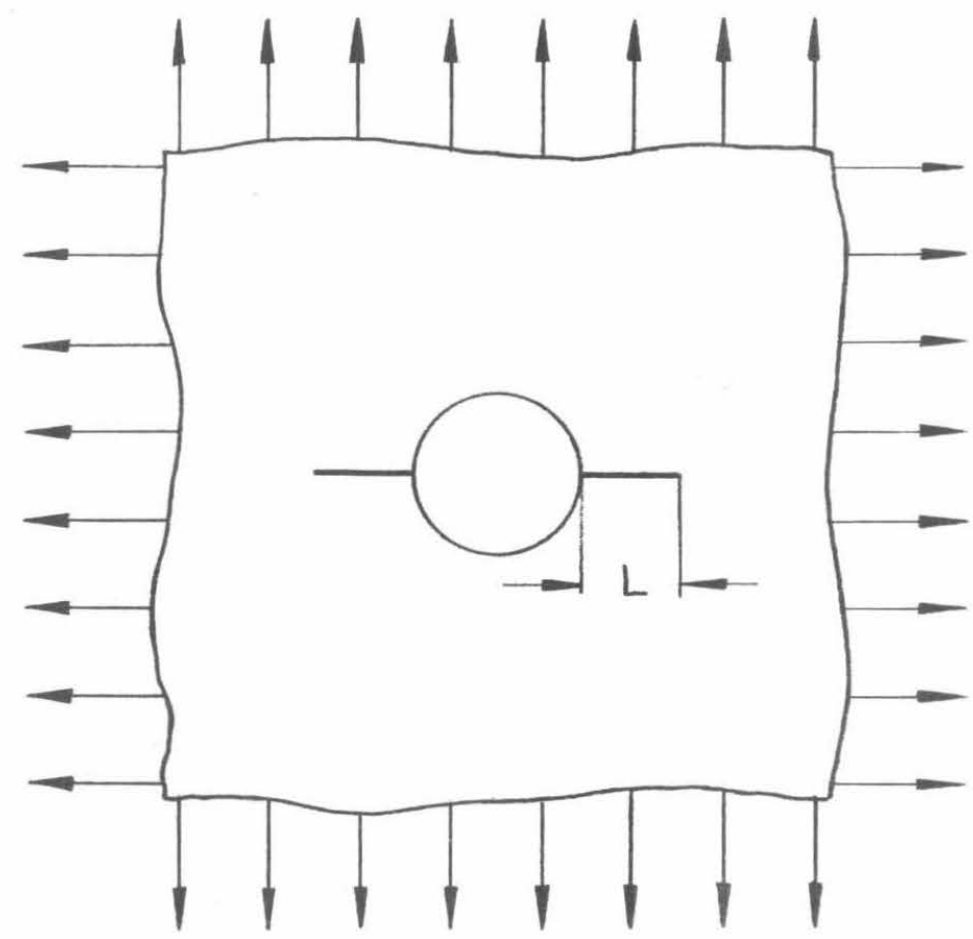

Figure 50. Two-Dimensional Saturn-Ring Crack 
In both instances the two-dimensional sheets are taken to be loaded in equal biaxial tension or what may be roughly referred to as a twodimensional hydrostatic tension. Griffith, (66) using the Inglis (80) solution for the line crack in a two-dimensional sheet, integrated the stresses and strains for a fixed force loading condition and gave as the strain energy of the body in plane strain the following:

$$
\mathrm{U}=\mathrm{U}_{0}+\frac{\pi\left(1-\nu^{2}\right) \mathrm{p}^{2} \mathrm{c}_{0}^{2}}{\mathrm{E}}
$$

Let us compare the form of this expression with one obtained from an approximate solution found by Bowie (81) for the two-dimensional Saturn-ring

$$
\mathrm{U}=\mathrm{U}_{0}+\frac{\pi\left(1-v^{2}\right) \mathrm{p}^{2} \mathrm{~L}^{2} \mathrm{k}(\mathrm{L})}{\mathrm{E}}
$$

$L$ is the half crack length as specified in Figure 54, and $k(L)$ can be given an analytic representation in three separate regions of the variable crack length $L$, by fitting Bowie's tabular data as is shown in Figure 51 .

$$
\begin{array}{ll}
\text { small } \mathrm{L} & \mathrm{k}(\mathrm{L})=2 \\
\text { intermediate } \mathrm{L} & \mathrm{k}(\mathrm{L})=\frac{2}{\mathrm{~L}^{2}}-\frac{1}{2}\left(1+\frac{1}{\mathrm{~L}}\right)^{2} \\
\text { large } \mathrm{L} & \mathrm{k}(\mathrm{L})=1
\end{array}
$$

From this $k(L)$ can be sketched over the entire range of $L$, and will appear as in Figure 52. 


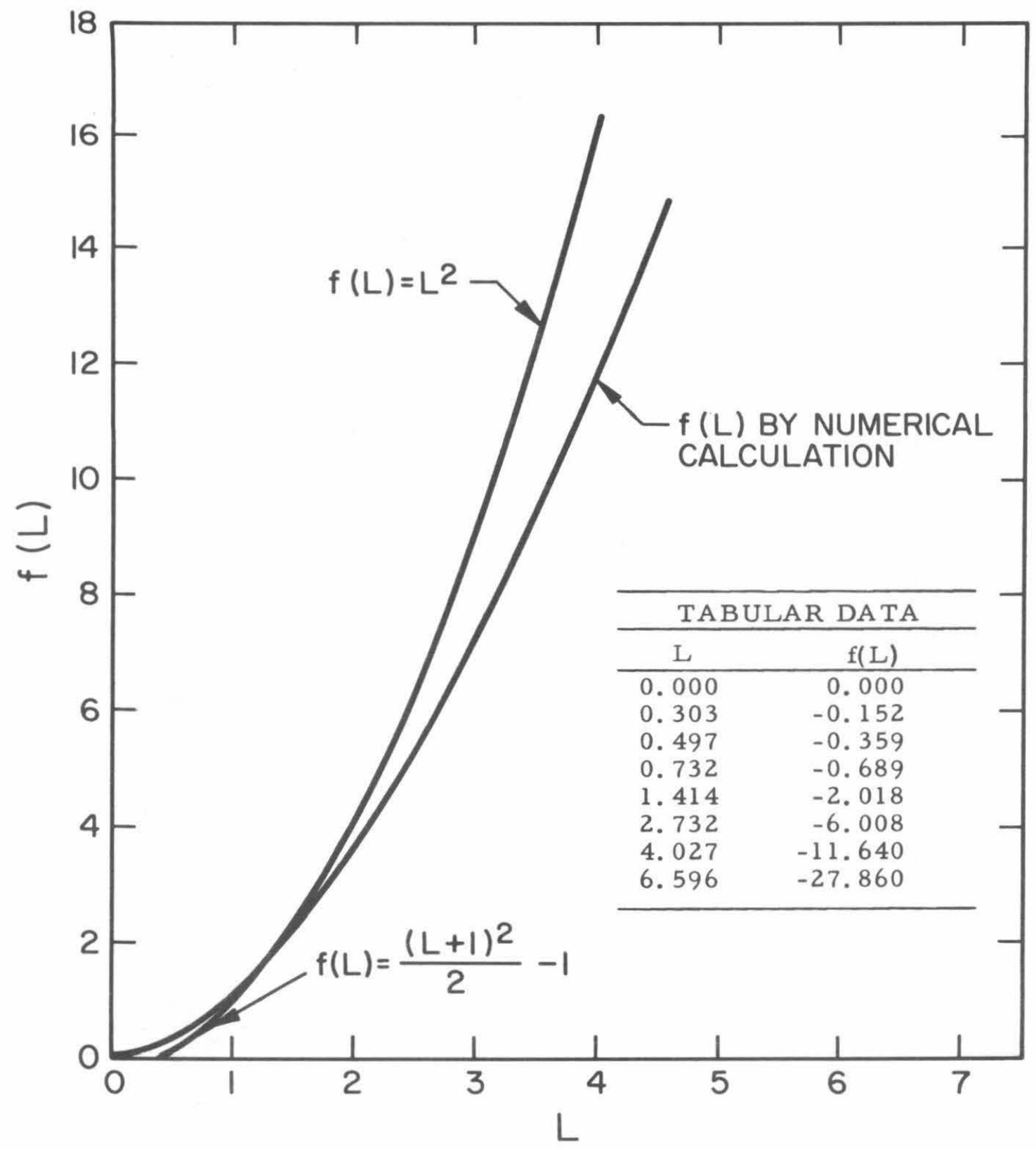

Figure 51. Plot of Bowie's ${ }^{(80)}$ Tabular Data Showing Regimes of Representation for $f(L)$. 


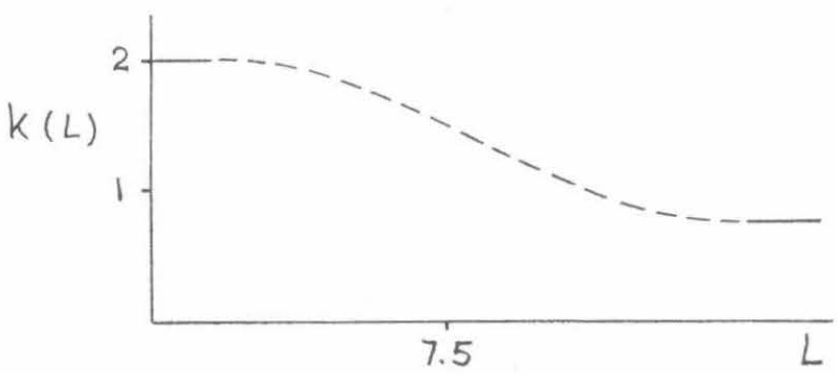

Figure 52. Crack Length Factor

The inflection point, $\mathrm{d}^{2} \mathrm{k} / \mathrm{dL}^{2}=0$, occurs at $L=7.5$. From this it is readily seen that there is not a large variation even when the entire range of $\mathrm{L}$ is considered. For the investigation of fracture initiation, the range for small $L$ is the one desired, and therefore $k(L)=2$ will be selected.

Having made the extension from the two-dimensional line crack to the two-dimensional Saturn-ring crack, we are now in a position to make a similar extension from a three-dimensional planar crack to a three-dimensional Saturn-ring crack. The planar crack, or so-called penny-shaped crack, shows almost no change from the line crack except in the dimensionality

$$
\begin{array}{lc}
\mathrm{U}=\mathrm{U}_{0}+\frac{\pi\left(1-v^{2}\right) \mathrm{p}^{2} \mathrm{c}_{0}^{2}}{\mathrm{E}} & \mathrm{U}=\mathrm{U}_{0}+\frac{8}{3} \frac{\left(1-v^{2}\right) \mathrm{p}^{2} \mathrm{c}_{0}^{3}}{\mathrm{E}} \\
\underline{\text { line crack }} & \text { planar crack }
\end{array}
$$

Using the previous example as a guide, it is conjectured that the strain energy of the three-dimensional Saturn-ring crack could be approximated as 


$$
\mathrm{U}=\mathrm{U}_{0}+\frac{8}{3} \frac{\left(1-v^{2}\right) \mathrm{p}^{2} \mathrm{~L}^{3} \mathrm{k}(\mathrm{L})}{\mathrm{E}}
$$

where $k(L)$ will be taken by analogy to be two for small crack lengths, and in order to limit check the penny-shaped crack solution for large L, where the spherical cavity should no longer have any effect, $k(L)$ must be equal to unity.

We have now closed a loop and have internal consistency in the ad hoc representation of the strain energy function. A schematic representation of this is given in Figure 53.

$$
\frac{\pi\left(1-\nu^{2}\right)}{E} p^{2} c^{2}
$$

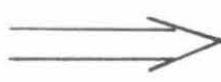

$\sqrt{ }$

$$
k(L)=2
$$$$
\frac{2 \pi\left(1-\nu^{2}\right)}{E} p^{2} L^{2}
$$
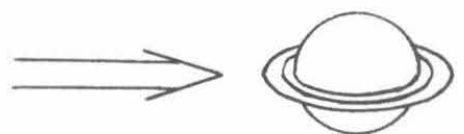

$\frac{16}{3} \frac{\left(1-\nu^{2}\right)}{E}$

Figure 53

The extended potential energy for the three-dimensional Saturn-ring crack can now be formulated ${ }^{*}$

The dependency on (c-a) must be as given in Eq. (5.4) in order to describe the planar mode of propagation, for a dependency of $\left(c^{3}-a^{3}\right)$ describes a volumetric change much the same as an ablating cavity rather than a propagating crack. 


$$
\bar{V}=-\left[U_{0}+\frac{16}{3} \frac{\left(1-v^{2}\right) p^{2}(c-a)^{3}}{E}\right]+2 \gamma \pi\left(c^{2}-a^{2}\right)
$$

where c represents the instantaneous location of the Saturn-ring crack measured from the center of the spherical cavity, and $\underline{a}$ is the radius of the cavity as shown in Figure 49.

\section{Criticality Condition}

Previously the point of instability, which located the critical stress at which the crack would run, has been found by setting the first variation of the extended potential energy equal to zero. There was also an alternate method discussed in Chapter IV on energy methods taken from Langhaar, (67) wherein the energy function was expanded in a Taylor series in terms of the variation parameter. In this case the extended potential energy $\bar{V}$ would be expanded in terms of the crack length $\mathrm{c}$ around an arbitrary point, say $\delta^{*}=\mathrm{a}+\mathrm{c}^{*}$, where $c^{*}$ is the radial length of some residual Saturn-ring crack. (See Figure 49.) The advantage of this method is that it provides better physical insight into the behavior during the variation process. Making such an expansion we obtain,

$$
\begin{aligned}
& \Delta \bar{V}=\bar{V}-V\left(\delta^{* *}\right)=\left[-16 \frac{\left(1-v^{2}\right)}{E} p^{2}\left(\delta^{* *}-a\right)^{2}+4 \gamma \pi \delta^{* k}\right]\left(c-\delta^{* *}\right) \\
& +\left[-32 \frac{\left(1-v^{2}\right)}{E} p^{2}\left(\delta^{* *}-a\right)+4 \gamma \pi\right] \frac{\left(c-\delta^{*}\right)^{2}}{2 !}-32 \frac{\left(1-v^{2}\right)}{E} p^{2} \frac{\left(c-\delta^{* k}\right)^{3}}{3 !}
\end{aligned}
$$

This equation can be qualitatively plotted to obtain a better picture of the interaction between applied load $\mathrm{p}$ and critical flaw size $\delta^{*}$ or $\mathrm{c}^{*}$. 


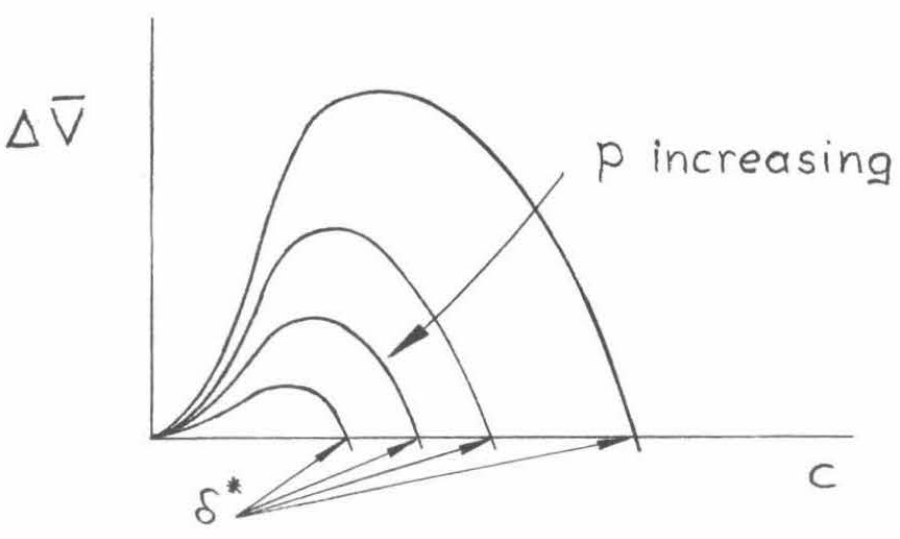

Figure 54. Adjusted Potential Energy vs. Crack Length

It was previously shown that $\Delta \overline{\mathrm{V}}$ was actually related to the slope; i.e., the first variation around this loaded state corresponded to a second variation around the unloaded state, and the point of interest is where the slope changes signs, or where the function $\Delta \overline{\mathrm{V}}$ crosses the horizontal axis. The actual point at which it crosses the axis is a function of the parameter $P$, and the point of crossing is $\delta^{*}$. If we investigate the function for values of crack length very near $\delta$, the first term is dominant, and the point at which the derivative changes sign can be closely approximated by setting the first term of equation $(5.5)$ equal to zero.

$$
\frac{4\left(1-v^{2}\right) p^{2}\left(\delta^{*}-a\right)^{2}}{E}=\gamma \pi \delta^{*}
$$

or

$$
\frac{4\left(1-v^{2}\right) p^{2}}{E \gamma \pi}=\frac{c^{*}+a}{c^{* 2}}
$$


Physical Interpretation

The physical interpretation of this variation or more specifically $c^{*}$, is not absolutely fixed, but it can be adapted to the physical processes involved in the fracture of $\mathrm{S}-113$. It is clear from Figure 54 that as $p$ increases, the residual Saturn-ring flaw c ${ }^{*}$ must decrease; however, in the limit as $c^{*}$ approaches zero, or in other words, in the limit of fracture of the spherical cavity alone without a Saturn-ring crack, the critical stress approaches infinity, as seen from (5.7) in the same manner as the original Griffith result. This is not actually true, for if one examines the atomic level, there is always a Saturn-ring flaw of the order of atomic dimensions, but this goes beyond the applicability of the continuum theory, and so it is appropriate to seek another interpretation of $c^{*}$.

By visual inspection of virgin S-113 under microscopic powers of $600 \mathrm{X}$ to $800 \mathrm{X}$, the continuum around the flaw appeared to be intact, and there was certainly no Saturn-ring flaw initially. However, it is believed from observing the experiments, that the actual fracture occurs in this Saturn-ring mode, from which it may be concluded that the material, after a fashion, grows its own flaw. To elaborate on this point, imagine that as an observer we are allowed to go inside the spherical cavity and watch the fracture as it initiates at that surface. As the load is applied at infinity, we would notice bonds beginning to break at various points on the surface where the stress is the highest; however after the first bond has broken, the material would still remain intact and fracture of the specimen would not occur. This bond breakage would not be 
discerned by an outside observer, for he would have no way of detecting it so we arrive at the tedious point of defining where fracture actually starts. If it is not defined to occur at the rupture of the first bond, is it at the point of the seventh bond, or at the onehundredth bond, or where is it?

Knauss (82) has devoted some attention to this problem as he has studied the propagation of cracks in sheet geometries at various velocities, and has arrived at this same conclusion - that a body will grow its own flaw. He has shown that for an H-C rubber, which is similar in many respects to S-113, the fracture surface characteristics change dramatically as the velocity changes, and that there are three distinct regions as he classified them. The first or slowest velocity regime is characterized by a very rough, cobbled surface where the fracture appears as if strands resembling rubber bands were pulled apart individually. This regime is for very low velocity crack propagation. The second regime, which he terms the transition region, is more smooth in appearance and much less cobbled. In the high velocity region, the fracture surface appears glassy and mirror-like. A plot of the crack propagation speed versus the gross stress applied to a sheet geometry is excerpted from this work (82) and shown in Figure 55. In that figure is contained the evidence of a very rapid change in velocity versus applied stress for certain ranges; in fact, in the slowly propagating regime, evidenced by a very rough surface, the velocity to a good approximation could be taken to be zero. It is this mechanism that is employed here to define $c^{*}$ and to interpret the manner in which 


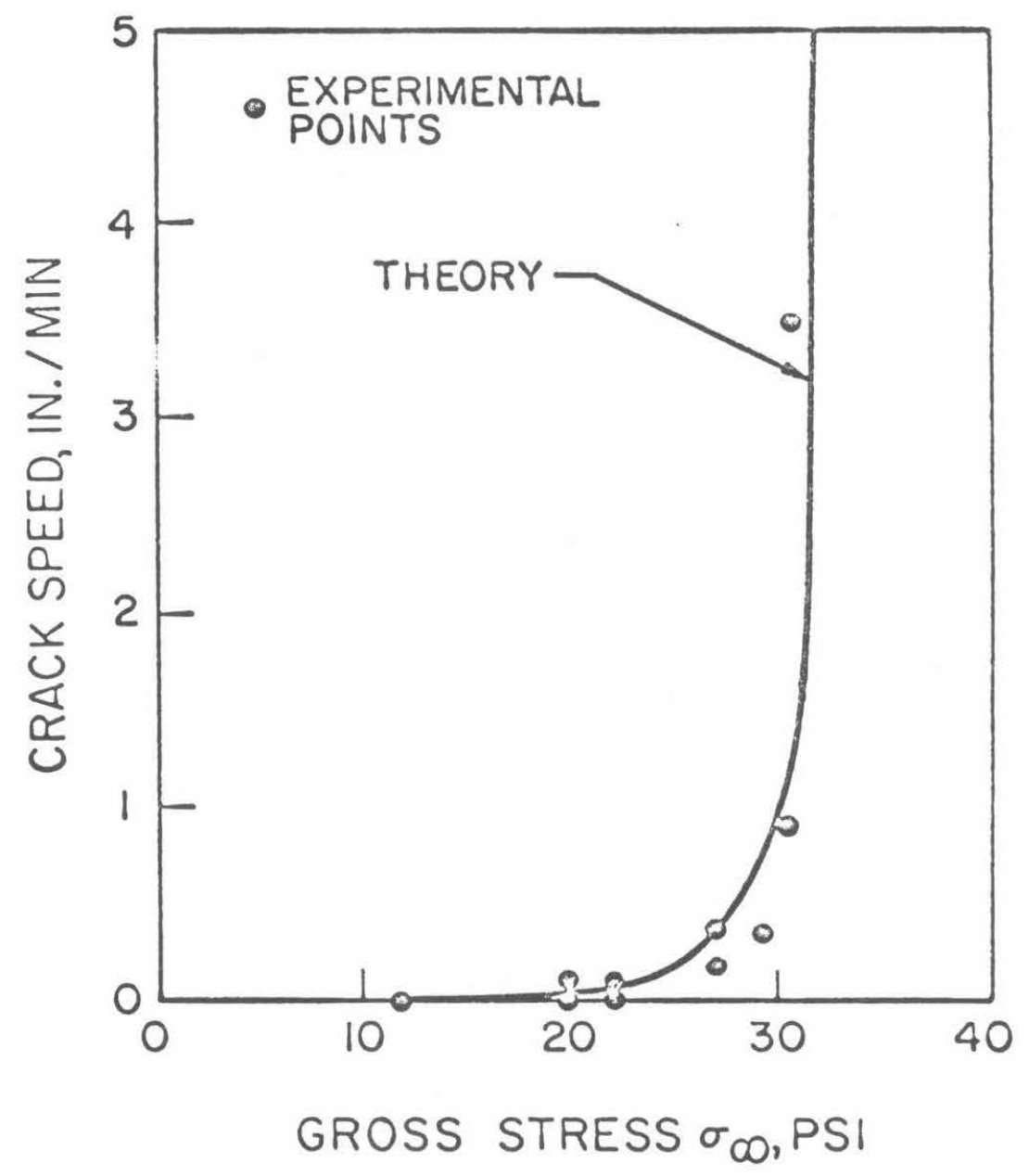

Figure 55. Crack Propagation Speed as a Function of Gross Stress in Strip Specimen. 
a body grows its own flaw.

Let us now return to the spherical cavity problem. As the stress increases to some limit point, a few bonds are broken and eventually as the stress is increased, enough broken bonds will occur in a localized region to create a microscopic crack, and this microscopic crack is propagating at almost zero velocity, as shown in Figure 55. But given enough time, the specimen will eventually rupture. Strictly speaking, the crack is unstable and the critical stress has been reached, but it goes undetected and may continue moving slowly for a long indefinite period. In this application the interest is in elastic fracture, and the rate effects can be ignored by assuming that the velocity of propagation is zero and that the body, as a result of the applied load, has created a microscopic flaw, which is perfectly stable and which will not run. As the load is increased further, the flaw will be made larger, but it will remain stable. Referring back to Figure 54, it is seen that the allowable flaw size is becoming smaller as the load is increased; thus the two variable parameters are approaching each other. As the applied hydrostatic pressure is increased, the critical flaw size that the body can withstand is decreased, but the actual flaw size is increasing at virtually zero velocity. Finally, the point will be reached where the flaw grown by the material becomes critical for the applied load and at that instant the flaw will become unstable, producing fracture of the entire specimen. Interpreted in light of the velocity results of Knauss, at that point the velocity will change from zero to the high velocity propagation level. It is at this point that 
fracture is defined by the observer on the outside of the specimen, for, by virtue of the energy released, a manifestation appears at the loading device of the testing machine, or by visual observation, the crack has become large enough that it can be observed as it. propagates. It is this interpretation that is given to $\mathrm{c}^{*}$. It is the size of a critical flaw grown during the loading process, emanating from the surface of the residual spherical cavity, and acting as a stress riser to produce fracture nucleation.

Limit Cases. It is convenient to cast equation (5.7) in terms of non-dimensional variables. Let us define $\xi=c^{*} / a$, then equation (5.7) becomes

$$
\mathrm{p}^{2} \sim \frac{\xi+1}{\mathrm{a} \xi^{2}}
$$

In the limit of $\xi$ becoming large, or where the parameter $c^{*}$ is large compared to the bubble radius $\underline{\text { a }}$,

$$
\mathrm{p}^{2} \sim \frac{1}{\mathrm{c}^{*}}
$$

which is a constant independent of the initial flaw size a. (It should be noted that it is not independent of the existence of a cavity - only its size.) Thus as the flaws become small compared to the fracture parameter $c^{*}$, they have no influence on the fracture and cause no damage to the material. They act only as nucleation points.

On the other hand, the limit of $\xi$ becoming small, where the cavity is the dominant factor

$$
\mathrm{p}^{2} \sim \frac{\mathrm{a}}{\mathrm{c}^{*}}
$$


and the large cavity causes an increase in the fracture strength over smaller cavities -- not over the perfect material. It behaves in much the same way that a large fillet radius does, causing a reduction in the stress concentration in a body and a consequent increase in strength. In the intermediate values, there is an interplay of the various parameters that can be qualitatively plotted as shown in Figure 56.

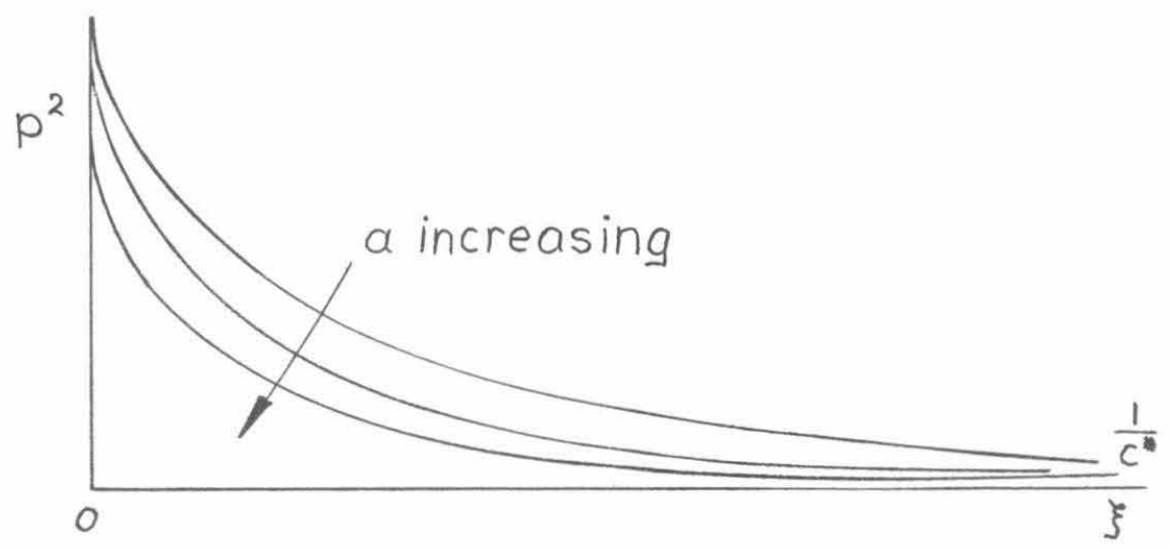

Figure 56. Critical Pressure vs. Flaw Size

This rather unusual result arising from the diameter term of equation (5.7) which indicates that the larger bubble will sustain more load before fracture was in keeping with the observed behavior. The theory predicts that for a fixed $c^{*}$, fracture would initiate at the smallest bubble in the matrix; however, in the poker-chip test there would be a trade-off between the size of the flaw and the location in the field due to the stress decreasing radially from the center. Consequently the actual pinpoint location would involve an interplay between the field stress and the size of the flaw. As a result, we have employed the average flaw size to compute the theoretical 
strength of the material, since this size flaw would have the highest probability of occurring at the point of maximum stress. However, statistical variations in cavity size and location would be partially responsible for strength variations. It would be predicted from equation (5.10) that if the larger artificially inserted bubble had been alone in the field, an appropriately larger fracture stress would have resulted; however the presence of the smaller cavities caused the fracture to nucleate from them and the fracture levels to remain unchanged, even with the presence of the large flaw.

Numerical Correlation. In order to demonstrate this theory further, a theoretical computation of strength will be made based on measurements of a flaw size a and $\delta$, taken from a typical specimen. From Figure $34, \delta^{*}$ is measured as $3.7 \times 10^{-4}$ in. Assuming that the fracture resulted from a typical cavity of radius $a=2 \times 10^{-4}, c^{*}$ is computed to be $1.7 \times 10^{-4}$ in. With these figures, all that is required to compute the critical stress is the surface energy density. This was measured in a separate experiment on a different configuration entirely. A two-dimensional sheet containing a crack was extended in plane stress. The configuration was adjusted to approximate a sheet of infinite extent with a semi-infinite crack, as shown in Figure 57.

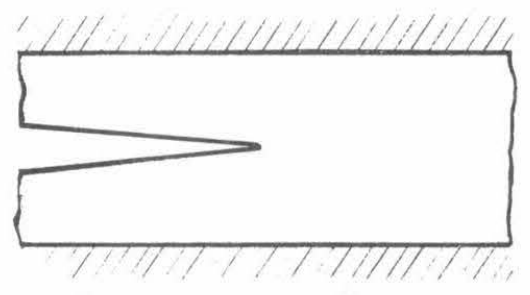

Figure 57. Sheet Configuration Used for Measuring Surface Energy 
By plotting the propagation velocity of the crack vs. the applied strain at the boundary, an approximate measure of the strain at which the crack propagates at virtually zero velocity can be obtained. At this point it is assumed that kinetic energy and dissipation are minimal; therefore the strain energy is directly converted to surface energy as the crack moves ahead. Equating the two, $\gamma$ comes out to be 0.05 in $1 \mathrm{~b} / \mathrm{in}^{2}$. The actual value for $\gamma$ is taken to be a material property independent of geometry and thus applicable in this analysis for HT.

Putting these values into equation (5.7) and using $\mathrm{E}=$ 500 psi and $v=\frac{1}{2}$

$$
\mathrm{p}_{\mathrm{Cr}}=580 \mathrm{psi}
$$

This is very near the recorded fracture value of 625 psi for the specimen from which these measurements were taken. Figure 29 is the apparent stress-strain curve for this specimen, which exhibits an apparent fracture strain of .026 in/in. From Figure 8, $\sigma_{\mathrm{z}} / \mathrm{E} \varepsilon=48$, where $\sigma_{\mathrm{z}}=\mathrm{p}_{\mathrm{Cr}}$, and the measured value of the local hydrostatic pressure is 625 psi. Such good agreement may be a bit fortuitous, but it lends support to the validity of the theory and calculations.

Thus the Saturn-ring concept, with the accompanying calculations, is able to predict experimental results as well as provide a plausible explanation for the observations made with the large, artificial flaws. The results forthcoming from the analysis are of considerable interest when discussing the 
difference between cavities and cracks. The energy formulations show that they enter the problem in a fundamentally different way, as seen by comparing equation (5.7) to Sneddon's result, for instance. It is true that for a crack the larger its size, the less its endurable load; however cavities do not act this way. The comparative results between the two continues to behave as intuitively expected; i.e., a body possessing a cavity, for a hole, of the same dimension as a crack would be expected to withstand more stress, or would bear more load than the cracked body and this is exactly what the energy expressions predict. The exact manner in which the cavity radius enters is extremely interesting. It shows that in some regimes the cavity is not important at all except in acting as a nucleation point and lends a new concept about the effect or residual cavities in rubbery materials, or other materials for that matter, and their effect upon the malfunctioning of that body. Heretofore it has been the intuitive feeling that has motivated quality control techniques, which accepts a structure that has flaws under a specified size and discards as inacceptable bodies containing flaws that would exceed this randomly selected size. This result will have significant meaning for people engaged in such work, for it demonstrates that another piece of information, $c *$, must be known before a decision can be made. It must be emphasized in conclusion that this application is for hydrostatic tension only and may be much different in another geometry; in fact, it will be demonstrated that this actually is the case in the next section. 


\section{RELATED GEOMETRIES}

Although the purpose of this study was to investigate hydrostatic fracture, the unusual nature of the cavity size dependency makes it intriguing to know what other geometries will yield. A brief look at the two-dimensional analogy, consisting of a cylindrical, hole in equal biaxial tension, provides additional understanding of the role of cavities as opposed to cracks. Returning again to the Bowie (81) solution, the expression for the extended potential energy becomes

$$
\bar{V}=U_{0}-\frac{2 \pi p^{2}}{E}(c-a)^{2}+4 \gamma(c-a)
$$

where $\mathrm{a}=$ radius of the original cylindrical hole

$c=$ length of the crack measured from the periphery of the hole

The critical condition obtained by expanding $\bar{V}$ in a Taylors Series about a given crack size $c^{*}$ is

$$
\mathrm{p}_{\mathrm{cr}}=\sqrt{\frac{\mathrm{E} \gamma}{\pi \mathrm{c}}}
$$

when $c^{*}$ is again taken as the crack dimension 'grown' in the material by the loading at zero velocity before the fracture process becomes unstable.

This is an interesting turn of events, because the initial hole size, a, does not appear explicitly. It does influence the expression for strain energy $(5.10)$, since this is dependent upon geometry; however the influence is the same regardless of the size of the hole. This statement should be tempered with the fact 
that the hole must be small compared to specimen dimension. Furthermore the theory assumes that fracture occurs simultaneously over the entire thickness of the specimen. Although this assumption is standard for the reason that the thickness effect is a separate study in and of itself, (83) it is exceptionally crucial in this instance. It directly affects the form of the surface energy expression, which is the term that gives rise to the appearance of the initial cavity size in the criticality condition. Within these limitations the fracture stress should be independent of initial hole, or cavity, size.

Experimental Results

A few preliminary tests have been run to evaluate this prediction. Only a small number of runs were made for equal, biaxial tension, but a significant number of specimens were tested in uniaxial tension. For the biaxial tests, specimens were prepared by casting large, circular sheets 13 inches in diameter and 0.10 inches thick in the standard way. After curing, the central portion of the sheet was frozen with liquid nitrogen, which made it possible to drill holes with smooth surfaces free of stress concentrations. The equal biaxial stress field was produced by inflating this membrane with the central cylindrical hole by a pressure apparatus similar to that of Figure 4. Loading the sheets to failure for a series of holes of $1 / 16,1 / 8$ and $1 / 4$ inches in diameter, fracture stresses of 109 psi, 123 psi, and 116 psi respectively were recorded. For all intents and purposes this can be considered constant. These experiments are by no means conclusive, but they do display the trend 
predicted by equation (5.12), and one statement that can be made is that the dependency of hole size does not appear to be inverse square root as is the case for cracks.

Of course, the same geometry loaded by a one-dimensional stress field does not possess fundamentally different energy expressions from the two-dimensional case; consequently, the critical pressure possesses the same form except for the magnitude of constants. A series of tests was run for uniaxial specimens containing cylindrical holes, ranging in size from 0.03 to 0.525 in., or over approximately a one decade spread. The specimens were $3^{\prime \prime} \times 4^{\prime \prime} \times 0.10^{\prime \prime}$ giving a specimen width to cavity ratio of the orders of 10 to 100. Results of the test are plotted in Figure 58, where a remarkable demonstration of the predicted result of equation (5.12) is manifested. The fracture level is constant except for a small transition region where the cavity sees a change from the plane stress environment to plane strain. In other words, when the hole size is large compared to the thickness it sees a plane stress field, but as the hole size is reduced, it finally becomes approximately the same magnitude as the specimen thickness. From this point on, the hole sees a plane strain field and the energy enters the process, as well as the fracture equation (5.10), in a slightly different way. The strain energy is altered by a factor of $1 / 1-\nu^{2}$ and the critical stress is increased by the square root of this quantity. For purposes of a nearly incompressible material such as S-113, the factor becomes 1.15. This is almost exactly the increase that is observed experimentally in Figure 58 as the 
-144-

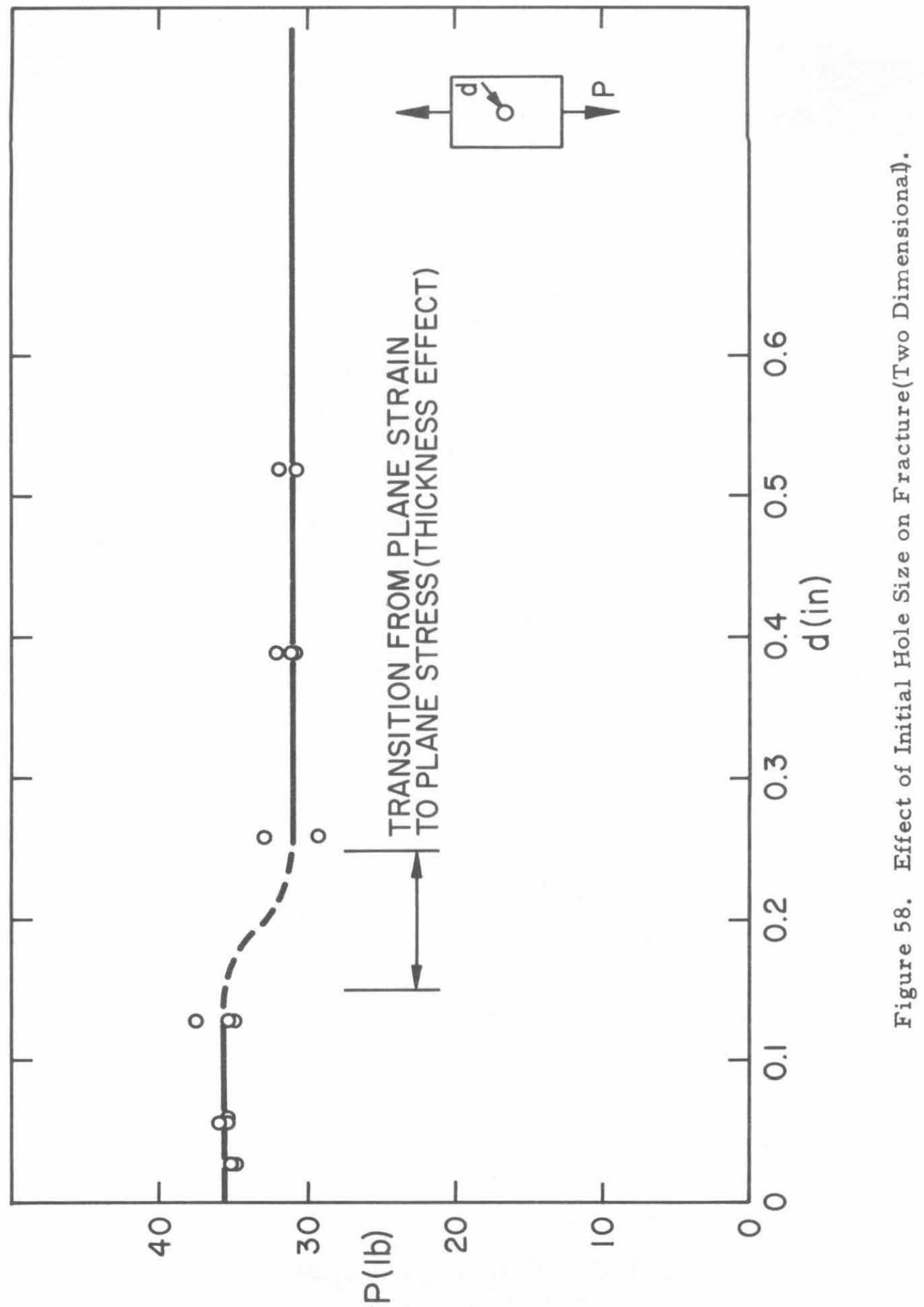


fracture level changes from 31.5 to 36.0 on the average.

This brief side excursion from hydrostatic fracture suggests additional corroboration of the concept of a flaw 'grown' by the loading, which concept appears to predict rather accurately the experimental results. It further suggests some alterations in one's intuition with regards to the effects of the dimensionality of the stress field and the influence of cavity size on fracture level. The general trends noted here can be nicely fit into the stress-axis theorem on fracture, alluded to previously, to provide guidelines for engineering analysis and design.

Character of $c^{*}$

One additional item of interest is the correlation of the concept of an actual numerical value of $c^{*}$ in the various stress fields. At this point it is not possible to deduce a dependency of $\mathrm{c}^{*}$ on geometry or material properties; i.e., whether it is predominantly determined by the geometry of a stress field or whether there is a fundamental mechanism tied up with the chain structure that dictates the magnitude of the flaw that must be grown before it becomes unstable and propagates at high velocity. Additional experimental work will have to be conceived and carried out to define the exact properties of $c^{*}$. Along this same line is the product of the material properties E $\gamma$ which always arise in any fracture analysis, indicating that a wide range of materials possessing a diversity of mechanical properties could still fracture at the same point if the $\mathrm{E} \gamma$ product remained the same. In other words a more glassy 
polymer possessing a high modulus may at the same time have a low surface energy density and still fracture at the same point as a rubbery polymer with a low modulus and a high surface energy density, providing $\mathrm{c} *$ is the same. This reasoning attributes additional importance to the fundamental nature of $\mathrm{c}^{*}$ as describing the difference in character of the fracture between two materials of this type--if there is a difference.

\section{SUMMARY}

In summary it is felt that the results of the poker-chip experiments on both the standard material and the material with the artificial flaws have amplydemonstrated that the mechanism of polymer fracture through a Saturn-ring crack, whereby the material generates its own flaw, is a reasonable one. Furthermore the preliminary results of the one and two-dimensional tests seem to attest the conclusion reached in the three-dimensional analysis that the hole, or cavity, size affects fracture properties in a manner completely different than a crack. Having touched all of the bases, so to speak, it appears that a consistent story has been generated that has been verified experimentally, from which extensions can now be made to the more general case where holes and cavities are analyzed in the viscoelastic range. 


\section{CHAPTER VI}

\section{CONCLUSION}

At this point it is appropriate to assemble in brief form the main contributions of this study in an effort to integrate the several parts into a whole, and to better delineate what progress has been made. A lot of groundwork has been laid in the form of experimental test development, specimen stress analysis, etc. that will continue to be useful in further triaxial fracture studies. Results of experimental tests employing these tools have provided quantitative definition of ultimate strengths in hydrostatic tension, which provides the limit point for the topological fracture surface in principal stress space.

The discovery, measurement and distribution analysis of spherical cavities in the material has given better insight to guide the theoretical treatment of flaws. Furthermore it has identified a type of material that can be used for extensive investigation of cavity flaw behavior. It may also lead to the identification of other materials of a similar classification, which possess inherent flaws due to manufacturing and processing. These steps forward all have applications beyond the scope of this particular study and may be readily employed in extensions of this work.

The application of energy methods of fracture analysis to spherical cavities has led to predictions of ultimate strengths, both for finite and infinitesimal deformations, which compare favorably with experiment in some regimes; however many of the same problems that plagued Griffith in his initial work with energy 
analysis still plague us today. By this it is meant that from the standpoint of fundamental physical processes involved, a great deal still remains to be learned. For instance Griffith (66) performed experiments on glass that he used as evidence of his theoretical predictions. In many respects they did not duplicate the conditions imposed in the mathematical treatment, and this continues to be, and promises to remain, a challenge to the experimentor. To accurately duplicate the ideal boundary conditions is a tedious and difficult task. In addition, he required the surface energy of the glass as a parameter in the expression for critical stress. Since he could not measure this quantity in the solid state, he used the liquid value as an approximation. In the intervening forty years since Griffith published his work, the colloidal chemists have been studying surfaces, or more accurately, interfaces, and have learned a great deal about them. Most of this knowledge, however, has pointed to the fact that it is a much more complicated phenomenon than had been originally appreciated, and the concept of surface energy in solids is still quite nebulous. So that just exactly what surface energy is in solids and the mechanisms involved in creating a surface during fracture still remain as missing pieces in the puzzle. Such key pieces are worthy of study and constitute a logical avenue of further research.

Another finding of this study relates to the difference in effect on the strength of a body containing cracks and cavities. Once again the confidence level in the results is limited by the lack of understanding of the basic processes. The energy approach used 
seems to be sound and well motivated mathematically; however the separate terms in the analytical expressions strongly depend upon the physical mechanisms and how accurately they can be described mathematically. In cracks this is a little better defined than in cavities, since propagation details can be formulated more readily on an intuitive basis. The manner in which a spherical cavity opens up into a crack is not at all obvious. As a result of this study, which has concentrated on discovering some of the fundamental mechanisms, additional insight into the behavior of cavities as fracture nucleation points has been obtained. However most of this information has been gleaned from evidence left on the fracture surface; consequently the hypothesis of the Saturnring propagation mode is predominantly inference and,although it seems to explain all of the phenomena, the evidence is still not conclusive. In other words, from almost any standpoint there is a need for extensive fundamental studies on elastic fracture processes. These items will also constitute a valuable contribution when dissipative effects of viscoelastic materials are included, or when dealing with the more complicated filled material. 


\section{REFERENCES}

1. P. W. Bridgman, "The Physics of High Pressure," G. Bell and Sons Ltd., London 1949.

2. P. W. Bridgman, "Studies in Large Plastic Flaw and Fracture," McGraw-Hill, New York, 1952.

3. P. J. Blatz, "Yield Surface in Normal Stress or Normal Strain Span," SM 60-14, Calif. Inst. Tech., August 1960. Published in JANAF Proceedings, September 1960.

4. A. C. Eringen, "Nonlinear Theory of Continuous Media," McGraw-Hill, New York, 1962.

5. L. J. Briggs, "The Limiting Negative Pressure of Acetic Acid, Benzene, Aniline, Carbon Tetrachloride and Chloroform," J. Chem. Phys., Vol. 19, 1951, p. 970.

6. J. C. Fisher, "The Fracture of Liquids," J. Applied Physics, Vol. 19, 1948, p. 1062 .

7. G. R. Irwin, "Fracture," in Handbuch der Physik, Vol. VI, P. 551, Springer-Verlag, Berlin, 1958.

8. M. Hetenyi, "Handbook of Experimental Stress Analysis," John Wiley and Sons, New York, 1950.

9. A. Nadai, "Theory of Flow and Fracture of Solids," Mc GrawHill, New York, 1950.

10. H. Neuber, "Theory of Notch Stresses, "J. W. Edwards Co., Ann Arbor, Michigan, 1946.

11. Lehrer and Schwalzbart, "Static and Fatigue Strength of Metals Subjected to Triaxial Stresses, "IAS paper no. 60-12, 1960. 12. D. J. McAdam, "Fracture of Metals Under Combined Stress," 
ASM Vol. 37, 1946, p. 538.

13. T. Alfrey, "Mechanical Behavior of High Polymers, "Interscience Publishers, New York, 1948.

14. F. Bueche, "Physical Properties of Polymers, "Interscience Publishers, New York, 1960.

15. P. J. Flory, "Principles of Polymer Chemistry," Cornell Univ. Press, Ithaca, New York, 1953.

16. M. L. Williams, "Structural Analysis of Viscoelastic Materials, AIAA, Vol. 2, No. 5, May 1964, pp. 785-809.

17. A. M. Bueche and J. P. Berry, "The Mechanism of Polymer Failure," in Conference on Fracture, held at Swampscott, Mass., 1959.

18. M. L. Williams, "Fracture of Viscoelastic Materials," in Fracture of Solids, Interscience Publishers, New York, 1963.

19. M. L. Williams, P. J. Blatz, R. A. Schapery, "Fundamental Studies Relating to Systems Analysis of Solid Propellants, SM 61-5, Calif. Inst. of Tech., February 1961.

20. R. F. Landel and R. F. Fedors, "Rupture of Amorphous, Unfilled Polymers, " in Fracture Processes in Polymeric Solids, Interscience Publishers, New York, 1964.

21. W. G. Knauss and E. Betz, "Some Characteristic Functions Pertinent to Failure of Viscoelastic Materials, " SM 65-16, Calif. Inst. of Tech. Published in Bulletin of 4th Annual Meeting of ICRPG Working Group on Mech. Behavior, Nov. 1965. 
22. W. L. Ko, Application of Finite Elastic Theory to the Behavior of Rubber-like Materials, "SM 63-13, Calif. Inst. of Tech., Ph.D. Thesis, 1963.

23. A. N. Gent and P. B. Lindley, "The Compression of Bonded Rubber Blocks," Journal of British Rubber Producers Research Association, Vol. 173, p. 111, 1959.

24. A. N. Gent and P. B. Lindley, "Internal Rupture of Bonded Rubber Cylinders in Tension," Proc. of Royal Society, Vol. 249A, 1959, p. 195.

25. F. L. Yerzley, "Industrial Engineering Chemistry," Vol. 31, 1939, p. 950.

26. R. Houwink, "The Strength and Modulus of Elasticity of Some Amorphous Materials Related to their Internal Structure," Trans. of Faraday Soc., Vol. 32, 1936, p. 122.

27. P. J. Flory, "Effects of Molecular Structure on Physical Properties of Butyl Rubber, "Industrial and Engineering Chemistry, Vol. 38, No. 4, 1946, p. 417.

28. P. J. Blatz, N. George, S. Kawabata, "Physico-Mechanical Behavior of Rubberlike Materials," MATSCIT-PS 65-a, Calif. Inst. of Tech., April 1965.

29. W. G. Knauss, "Rupture Phenomena in Viscoelastic Materials", SM 63-10, Calif. Inst. of Tech., Ph.D. Thesis, 1963.

30. G. H. Lindsey, "Energy Criteria for Elastic Fracture, SM 65-20, Calif. Inst. of Tech., August 1965.

31. J. D. Ferry, "Viscoelastic Properties of Polymers," John Wiley and Sons, New York, 1961. 
32. L. R. G. Treloar, "The Physics of Rubber Elasticity," Oxford Press, London, 1958.

33. R. A. Schapery, "An Engineering Theory of Nonlinear Viscoelasticity with Applications," A and ES 65-5 Purdue Univ., June 1965.

34. G. Pickett,"Application of the Fourier Method to the Solution of Certain Boundary Problems in the Theory of Elasticity," Journal of Applied Mechanics, Vol. 11, Sept. 1944, p. A-176.

35. A. R. Zak and M. L. Williams, "Crack Point Stress Singularities at a Bimaterial Interface," SM 62-1, Calif. Inst. of Technology, 1962 .

36. E. C. Francis and D. Cantey, "Structural Integrity of Prope1lant Grains," Lockheed Propulsion Company, Report No. $556-\mathrm{F}$, January 1963.

37. M. L. Williams and R. A. Schapery, "Studies of Viscoelastic Media," Aeronautical Research Laboratories, WrightPatterson Air Force Base, Ohio, ARL 62-366, June 1962.

38. A. N. Gent, Proceedings of the Rubber Engineers Conference, 1956, p. 25 .

39. A. M. Messner, "Stress Distribution in Poker Chip Tensile Specimen," Second Meeting Bulletin of ICRPG Working Group on Mechanical Behavior, CPIA Pub. No. 27, Oct. 1963, p. 109 .

40. J. J. Brisbane, "Stresses, Strains and Displacements in the Pokerchip Test Specimen, "Report No. P-63-13, Quarterly Progress Report on Engineering Research, Rohm and Haas 
Co., Huntsville, Ala., July 1963.

41. G. H. Lindsey, R. A. Schapery, M. L. Williams, A. R. Zak, "The Triaxial Tension Failure of Viscoelastic Materials," Aerospace Research Laboratories Report ARL 63-152, September 1963.

42. M. L. Williams, "Surface Stress Singularities Resulting from Various Boundary Conditions in Angular Corners of Plates under Bending," Proceedings First National Congress Applied Mechanics, June 1951.

43. M. L. Williams, "Stress Singularities Resulting from Various Boundary Conditions in Angular Corners of Plates in Extension," Journal of Applied Mechanics, Vol. 74, 1952, p. 526.

44. M. L. Williams, "The Stresses Around a Fault or Crack in Dissimilar Media, "Bulletin of the Seismological Society of America, Vol. 49, No. 2, April 1959, p. 199.

45. A. R. Zak, "Stress Singularities in Bodies of Revolution," SM 62-29, Calif. Inst. of Tech., July 1962.

46. G. H. Lindsey and A. R. Zak, "Continued Studies of Stress Singularities in Bi-Material Media." GALCIT SM 62-52, Calif. Inst. of Tech., Dec. 1962.

47. I. S. Sokolnikoff, "Mathematical Theory of Elasticity," McGraw-Hill, New York, 1956.

48. G. H. Lindsey, "Stress Distribution in a Pokerchip Specimen Subjected to Combined Loads," GALCIT SM 64-23, Calif. Inst. of Tech., Published in Bulletin of Third Annual Meeting of ICRPG Solid Propellant Working Group on Mechanical 
Behavior, Oct. 1964.

49. S. Timoshenko and J. N. Goodier, "Theory of Elasticity," McGraw-Hill, New York, 1951.

50. W. G. Knauss, "A Cross-Linked Polymer Standard Report on Polymer Selection, "MATSCIT Report PS 65-3, Calif. Inst. of Tech., April 1965.

51. A. R. Zak, "Multiaxial Failure of Viscoelastic Materials," Aerospace Research Laboratories ARL 64-144, Sept. 1964.

52. M. L. Williams, R. J. Arenz, "The Mechanical and Optical Characterization of Solithane 113," Second International Congress on Experimental Mechanics, Washington, D. C., Sept. 1965.

53. W.G. Knauss, "A Cross-Linked Polymer Standard Report on Polymer Selection," MATSCIT Report PS 65-3, Calif. Inst. of Tech., April 1965.

54. A.R. Zak, "Uniaxial Characterization of Solithane 113 Viscoelastic Properties," GALCIT SM 64-37, Calif. Inst. of Tech., Aug. 1964.

55. M. L. Williams, R. F. Landel and J. D. Ferry, J. American Chemical Society, Vol. 77, 1955, p. 3701.

56. R. J. Arenz, C. W. Ferguson, T. Kunio, M. L. Williams, "The Mechanical and Optical Characterization of Hysol 8705 with Application to Photoviscoelastic Analysis," Tech. Documentary Report No. WLTDR 64-4, June 1964.

57. T. L. Smith, "Stress-Strain-Time Temperature Relationship for Polymers," Reprint from Symposium on Stress-Strain- 
Time Temp. Relationships in Materials, Special Technical Publication No. 325, ASME.

58. S. W. Beckwith and G. H. Lindsey, "Finite Strain Characterization of Solithane 113," GALCIT SM 65-15, Calif. Inst. of Tech., August 1965.

59. D. F. Miner and J. B. Seastone, "Handbook of Engineering Materials," John Wiley and Sons, New York, 1955.

60. B. C. Harbert, "Triaxial Tensile Failure of Solid Propellants," Bulletin of Third Annual Meeting of ICRPG Working Group on Mechanical Behavior, Vol. I, November 1964.

61. M. Levinson and P. J. Blatz, "The Finite Plane Strain Deformation of a Thin Pad." GALCIT SM 64-5, Calif. Inst. of Tech., March 1964.

62. E. Betz, "Some Mechanical Properties of Solithane 113," GALCIT SM 65-12, Calif. Inst. of Tech., July 1965.

63. W. G. Knauss, "The Time Dependent Fracture of Viscoelastic Materials," GALCIT SM 64-47, Calif. Inst. of Tech. Preprint International Conference on Fracture, Sendai, Japan, Sept. 1965.

64. W. G. Knauss, "On the Mechanical Properties of an H-C Rubber, Bulletin of Second Mtg. of ICRPG Working Group on Mechanical Behavior, Oct. 1963, p. 179.

65. E. Orowan, "Fracture and Strength of Solids, "In Report on Progress in Physics, Physical Soc. of London, Vol. 12, 1949, p. 185 .

66. A. A. Griffith, "The Phenomena of Rupture and Flow in Solids," 
Phil. Trans. of Royal Society, Series A, Vol. 221, 1921.

67. H. L. Langhaar, "Energy Methods in Applied Mechanics," John Wiley and Sons, New York, 1962.

68. L. A. Pars, "On Introduction to the Calculus of Variations," John Wiley and Sons, New York, 1962.

69. A. E. H. Love, "The Mathematical Theory of Elasticity," Oxford Press, London, 1927.

70. I. N. Sneddon, "The Distribution of Stress in the Neighborhood of a Crack in an Elastic Solid," Proc. Royal Society, Series A, Vol. 187, 1946, p. 229.

71. R. A. Sack, "Extension of Griffith's Theory of Rupture to Three Dimensions," Proc. Physical Soc. of London, Vol. 58,1946, p. 729.

72. A. A. Griffith, "The Theory of Rupture," Proceedings of the First International Congress for Applied Mechanics, 1924.

73. M. L. Williams, "Initiation and Growth of Viscoelastic Fracture," GALCIT SM 65-17, Calif. Inst. of Tech., Sept. 1965.

74. M. L. Williams and R. A. Schapery, "Spherical Flaw Instability in Hydrostatic Tension, "International Journal of Fracture Mechanics, Vol. No. 1, March 1965.

75. A. E. Green and W. Zerna, "Theoretical Elasticity," McGrawHill, New York, 1951.

76. M. Levinson, "The Complementary Energy Theorem in Finite Elasticity," Clarkson College of Tech., Potsdam, New York.

77. G. H. Lindsey, "Comparative Study of Hydrostatic Fracture for Neo Hookean and Mooney-Rivlin Materials, " GALCIT 
SM 65-24, Calif. Inst. of Tech., Nov. 1965

78. M. Levinson, "The Finite Strain Behavior of Incompressible Hollow Cylinders and Spheres under Internal Pressure," GALCIT SM 62-48, Calif. Inst. of Tech., Dec. 1962.

79. Schwarzl and Staverman

G. H. Lindsey, "Fracture Stress and Strength of High Polymers," GALCIT SM 64-44, CIT, December 1964. Translation of article by F. Schwarzl and A. J. Staverman, Die Physik der Hochpolymeren, Vol. IV, Springer-Verlag, 1956.

80. C. E. Inglis, "Stresses in a Plate Due to the Presence of Cracks and Sharp Corners," Proc. Institute of Naval Architects, March 14, 1913.

81. O. L. Bowie, "Analysis of an Infinite Plate Containing Radial Cracks Originating at the Boundary of an Internal Circular Hole," Journal Math. Phys., 35, 60, 1956.

82. W. G. Knauss, "The Time-Dependent Fracture of Viscoelastic Materials," GALCIT SM 64-47, CIT, December 1964. Presented at International Conference on Fracture, Sendai, Japan, September 1965 (to appear in Proceedings of ICF).

83. J. L. Swedlow, "The Thickness Effect and Plastic Flow in Cracked Plates," GALCIT SM 65-13, CIT, April 1965. Thesis. 
DOCUMENT CONTROL DATA - R\&D

(Security classification of title, body of abstract and indexing annotation must be entered when the overall report ie classified)

1. ORIGINATING ACTIVITY (Comporate author)

Graduate Aeronautical Laboratories

California Institute of Technology

Pasadena, California 91109

3. REPORT TITLE

Hydrostatic Tensile Fracture of a Polyurethane Elastomer

4. DESCRIPTIVE NOTES (Type of roport and inclusivo datos)
Scientific Report Oinal October 1964 - October 1965
5. AUTHOR(S) (Last name, first name, initial)
Lind sey, Gerald $\mathrm{H}$.

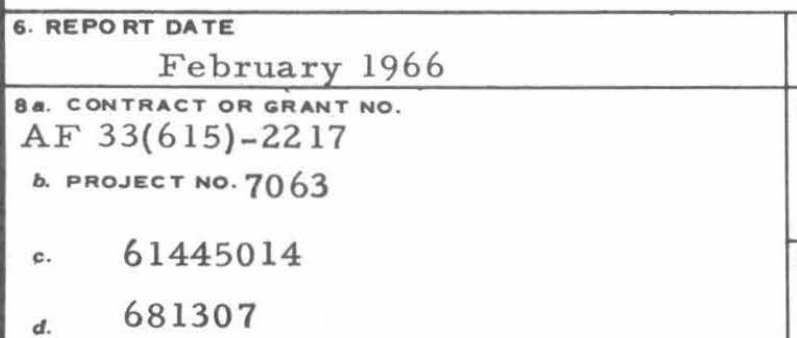

\begin{tabular}{|c|c|}
$\begin{array}{c}\text { 7a. TOTAL NO. OF PAGES } \\
158\end{array}$ & $\begin{array}{c}\text { 7b. NO. OF REFS } \\
83\end{array}$ \\
\hline
\end{tabular}

GALCIT SM 65-25

9b. OTHER REPORT NO(S) (A ny other numbere that may be anailened

ARL 66-0029

10. AVAILABILITY/LIMITATION NOTICES

1. Distribution of this document is unlimited.

Qualified requesters may obtain copies of this report from DDC.

11. SUPPLEMENTARY NOTES

12. SPONSORING MILITARY ACTIVITY

Aerospace Research Laboratories (OAR)

Office of Aerospace Research, USAF

Wright-Patterson AFB, Ohio

13. ABSTRACT The investigation of fracture of polymeric materials in hydrostatic tensile fields constitutes an avenue of approach to the study of fracture in more general three-dimensional environments. The advantages created by the symmetry of the stress field are considerable and, on one of the cases studied, facilitates a theoretical treatment that includes large deformations, which are characteristic of this class of materials. The analysis is developed through the concept of fracture originating from a flaw, which in this instance is taken to be a spherical cavity. Through the application of energy principles, a theoretical prediction of ultimate strength is made for hydrostatic tensile fields. Experiments were conducted to demonstrate the existence of such flaws and to evaluate the theory. Results of the tests on specimens containing both residual flaws and artificially inserted ones indicate a fundamental difference in behavior as contrasted with cracks. An explanation is given linking experimental results and theoretical predictions. It is based on the concept that a flaw "grows" in the material under load using the cavity as a nucleating point. Upon this hypothesis is built a theory of rupture in which planar cracks grow radially from the center of the cavity in the form of Saturn-ring cracks. 


\begin{tabular}{|l} 
14. \\
KEY woros \\
\hline Fracture in Polymers \\
Hydrostatic Tensile Fields \\
Theory of Large Deformations \\
Experimental Fracture Results \\
Rupture \\
Saturn-ring Cracks \\
Elastomers \\
Solid Propellant Integrity
\end{tabular}

\section{INSTRUCTIONS}

1. ORIGINATING ACTIVITY: Enter the name and address of the contractor, subcontractor, grantee, Department of Defense activity or other organization (corporate author) issuing the report.

2a. REPORT SECURTY CLASSIFICATION: Enter the overall security classification of the report. Indicate whether "Restricted Data" is included. Marking is to be in accordance with appropriate security regulations.

2b. GROUP: Automatic downgrading is specified in DoD Directive 5200.10 and Armed Forces Industrial Manual. Enter the group number. Also, when applicable, show that optional markings have been us ed for Group 3 and Group 4 as authorized.

3. RFPORT TITLE: Enter the complete report title in all capital letters. Titles in all cases should be unclassified. If a meaningful title cannot be selected without classification, show title classification in all capitals in parenthesis immediately following the title.

4. DESCRIPTIVE NOTES: If appropriate, enter the type of report, e.g., interim, progress, summary, annual, or final. Give the inclusive dates when a specific reporting period is covered.

5. AUTHOR(S): Enter the name(s) of author(s) as shown on or in the report. Enter last name, first name, middle initial. If rilitary, show rank and branch of service. The name of the principal aithor is an absolute minimum requirement.

6. REPORT DATE: Enter the date of the report as day, month, year, or month, year. If more than one date appears on the report, use date of publication.

7a. TOTAL NUMBER OF PAGES: The total page count shouid follow normal pagination procedures, i. e., enter the number of pages containing information.

7b. NUMBER OF REFERENCES Enter the total number of references cited in the report.

8a. CONTRACT OR GRANT NUMBER: If appropriate, enter the applicable number of the contract or grant under which the report was written.

$8 b, 8 c, 868$. PROJECT NUMBER: Enter the appropriate military department identification, such as project number. subproject number, system numbers, task number, etc.

9a. ORIGINATOR'S REPORT NUMBER(S): Enter the official report number by which the document will be ident ified and controlled by the originating activity. This number must be unique to this report.

9b. OTHER REPORT NUMBER(Ș): If the report has been assigned any other report numbers (either by the originator or by the sponsor), also enter this number(s).

10. AVAILABILITY/LIMITATION NOTICES: Enter any limitations on further dissemination of the report, other than those imposed by security classification, using standard statements such as:

(1) "Qualified requesters may obtain copies of this report from DDC."

(2) "Foreign announcement and dissemination of this report by DDC is not authorized."

(3) “U. S. Government agencies may obtain copies of this report directly from DDC. Other qualified DDC users shall request through

(4) "U. S. military agencies may obtain copies of this report directly from DDC. Other qualified users shall request through ,

(5) "All distribution of this report is controlled. Qualified DDC users shall request through "

If the report has been furnished to the Office of Technical Services, Department of Commerce, for sale to the public, indicate this fact and enter the price, if known.

11. SUPPLEMENTARY NOTES: Use for additional explanatory notes.

12. SPONSORING MILITARY ACTIVITY: Enter the name of the departmental project office or laboratory sponsoring (pay ing for) the research and development. Include address.

13. ABSTRACT: Enter an abstract giving a brief and factual summary of the document indicative of the report, even though it may also appear elsewhere in the body of the technical report. If additional space is required, a continuation sheet shall be attached.

It is highly desirable that the abstract of classified reports be unclassified. Each paragraph of the abstract shall end with an indication of the military security classification of the information in the paragraph, represented as (TS), (S), (C), or (U).

There is no limitation $\mathrm{cn}$ the length of the abstract. However, the suggested length is from 150 to 225 words.

14. KEY WORDS: Key words are technically meaningful terms or short phrases that characterize a report and may be used as index entries for cataloging the report. Key words must be selected so that no security classification is required. Identifiers, such as equipment model designation, trade name, military project code name, geographic location, may be used as key words but will be followed by an indication of technical context. The assignment of links, rules, and weights is optional. 